\title{
ESTUDO DE PLANEJAMENTO DE REATIVOS EM SISTEMAS ELÉTRICOS DE POTÊNCIA
}

\section{THALES SOUSA}

Dissertação apresentada à Escola de Engenharia de São Carlos da Universidade de São Paulo, como parte dos requisitos para obtenção do título de Mestre em Engenharia Elétrica.

ORIENTADOR: Prof. Dr. Geraldo R. M. da Costa

São Carlos

2003 
Aos meus Pais, incentivadores e responsáveis por todas as minhas conquistas. 
"Tente, Levante tua mão sedenta e recomece a andar não pense que a cabeça agüenta se você parar.

Queira, e basta ser sincero e desejar profundo você será capaz de sacudir o mundo.

Tente, não diga que a vitória está perdida se é de batalhas que se vive a vida Tente outra vez." 
Ao Prof. Dr. Geraldo Roberto Martins da Costa pela amizade, compreensão, dedicação e paciência desprendida durante a elaboração deste trabalho.

Aos companheiros do LOSEP: Alessandra, Cristiano, Edmarcio, e Vanusa pelo apoio, amizade e paciência.

Aos meus pais Vicente de Paulo e Geralda, ao meu irmão Júlio e a minha esposa Thaís por todo apoio nos momentos de maiores dificuldades e confiança em todas as minhas ações.

A todos os colegas, professores e funcionários do Departamento de Engenharia Elétrica da EESC/USP pela colaboração.

Ao Conselho Nacional de Desenvolvimento Científico e Tecnológico CNPq, pela bolsa de estudos. 
LISTA DE FIGURAS .................................................................... vii

LISTA DE TABELAS......................................................................... viii

LISTA DE ABREVEATURAS E SIGLAS.................................................

LISTA DE SÍMBOLOS........................................................................ xii

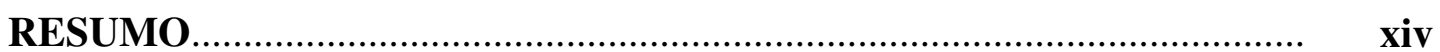

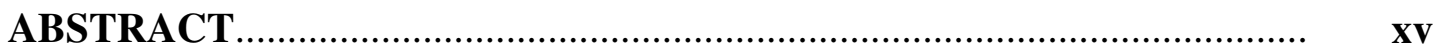

1 - INTRODUÇÃO

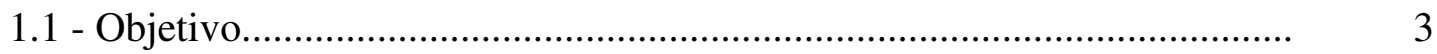

1.2 - Organização do Trabalho.............................................................................

2 - ESTADO DA ARTE DO PROBLEMA DE ALOCAÇÃO DE

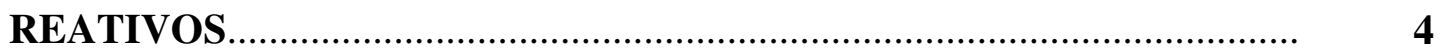

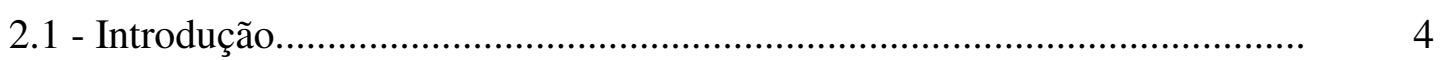

2.2 - Histórico...............................................................................................

3 - O PROBLEMA DE FLUXO POTÊNCIA ÓTIMO_.................................. 19

3.1 - Introdução........................................................................................... 19

3.2 - O Problema de Fluxo de Potência Ótimo........................................................ 20

3.3 - O Método Primal-Dual Barreira Logarítmica associada ao problema de Fluxo de Potência Ótimo...................................................................................... 23

3.3.1 - O Método de Newton............................................................................. 25

3.3.2 - Matriz Lagrangiana................................................................................ 27

3.3.3 - Tamanho do Passo e Atualização das Variáveis...................................... 27

3.3.4 - O Parâmetro de Barreira........................................................................... 29

3.3.5 - Inicialização das Variáveis..................................................................... 30

4 - FormulaÇão do PROBlema de Planejamento de REATIVOS EM SISTEMAS ELÉTRICOS DE POTÊNCIA....................... 31 
4.1 - Introdução....................................................................................... 31

4.2 - Escolha das Barras Candidatas à Alocação de Reativos........................... 33

4.3 - O Problema de Fluxo de Carga.............................................................. 34

4.4 - Obtenção da Matriz Sensibilidade.......................................................... 36

4.5 - Formulação do Problema de Programação Linear...................................... 38

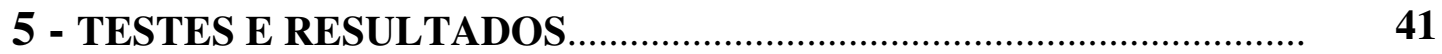

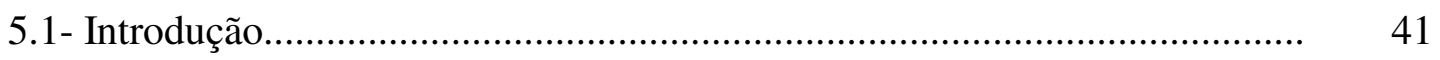

5.2 - Sistema WARD \& HALE.................................................................. 42

5.3 - Sistema de 8 barras............................................................................. $\quad 50$

5.4 - Sistema AEP - 30 barras......................................................................... 56

5.5 - Sistema IEEE - 118 barras.................................................................... 60

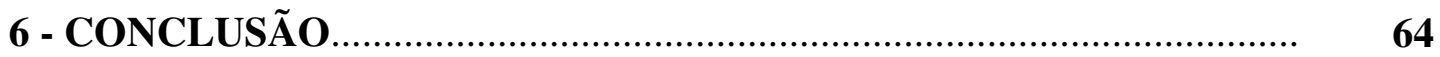

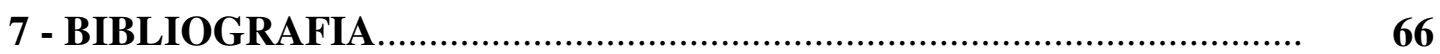

APÊNDICE 1 - Programação Linear - Método do Simplex

APÊNDICE 2 - Banco de Dados dos Sistemas Elétricos

APÊNDICE 3 - Estado Final dos Sistemas Elétricos 


\section{LISTA DE FIGURAS}

FIGURA 1 - Sistema WARD \& HALE ……................................................. 42

FIGURA 2- Gráfico representando os testes realizados segundo abordagem proposta 48

FIGURA 3- Sistema 8 barras ....................................................................... $\quad 50$

FIGURA 4- Sistema 30 barras ......................................................................... 56

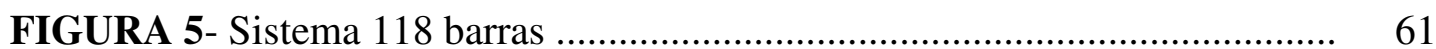


TABELA 1 - Dados iniciais do sistema WARD \& HALE

TABELA 2 - Ponto de operação do sistema WARD \& HALE, resultante do fluxo de carga convencional

TABELA 3 - Resultados do sistema WARD \& HALE para alocação reativa nas barras de carga do sistema

TABELA 4 - Solução do sistema WARD \& HALE resultante do Fluxo de Potência Ótimo

TABELA 5 - Resultados do sistema WARD \& HALE para alocação reativa na barra 5 do sistema

TABELA 6 - Solução do sistema WARD \& HALE resultante do Fluxo de Potência Ótimo após a injeção de reativos na barra 5

TABELA 7 - Resultados do sistema WARD \& HALE para alocação reativa na barra 3 e 5 do sistema

TABELA 8 - Dados iniciais do sistema de 8 barras

TABELA 9 - Ponto de operação do sistema de 8 barras resultante do fluxo de carga convencional

TABELA 10 - Resultados do sistema de 8 barras para alocação reativa nas barras de carga do sistema

TABELA 11 - Solução do sistema de 8 barras, resultante do Fluxo de Potência Ótimo

TABELA 12 - Resultados do sistema de 8 barras para alocação reativa na barra 8 do sistema

TABELA 13 - Ponto de operação do sistema AEP - 30 barras, resultante do fluxo de carga convencional

TABELA 14 - Resultado do sistema AEP - 30 barras para a alocação reativa nas barras de carga do sistema 
TABELA 15 - Resultado do sistema AEP - 30 barras para a alocação reativa nas barras determinadas pela abordagem proposta

TABELA 16 - Resultado do sistema IEEE-118 barras para a alocação reativa nas barras de carga do sistema

TABELA 17 - Resultado do sistema IEEE-118 barras para a alocação reativa nas barras determinadas pela abordagem proposta 


\section{LISTA DE ABREVIATURAS E SIGLAS}

p.u. - por unidade;

PLS - programação linear seqüencial;

PLI - programação linear inteira;

IEEE - Institute of Electrical and Electronics Engineers;

MPI - método dos pontos interiores;

Min - minimizar;

MAX - maximizar;

s.a. - sujeito a;

NB - número de barras do sistema elétrico;

NBC - número de barras de carga;

NBCR - número de barras de controle de reativo;

NBCCR - número de barras de carga e de controle de reativos;

NT - número de transformadores;

MPDBL - método primal-dual barreira logarítmica;

La - matriz Lagrangiana;

$\mathrm{J}$ - matriz Jacobiana;

$\alpha_{p}$ - passo primal;

$\alpha_{d}$ - passo dual;

$\mathrm{H}, \mathrm{M}, \mathrm{N}, \mathrm{L}$ - blocos da matriz Jacobiana;

$\mathrm{S}$ - matriz de sensibilidade;

W\&H- sistema WARD \& HALE;

Mbytes - Mega bytes;

RAM - Random Access Memory;

MHz - Mega Hertz;

LOSEP - Laboratório de Otimização em Sistemas Elétricos de Potência;

EESC - Escola de Engenharia Elétrica de São Carlos;

USP - Universidade de São Paulo;

MW - Mega Watts; 
MVA - Mega Volts Ampere;

MVAr - Mega Volt Ampere Reativo;

VAr - Volt Ampere Reativo;

LINDO - Linear Interactive Discrete Optimizer;

API - Application Programming Interface; 
$\bar{X}$ - limite máximo da grandeza $X$;

$\underline{X}$ - limite mínimo da grandeza $X$;

$\mathrm{V}$ - vetor das magnitudes das tensões;

$\theta$ - vetor dos ângulos das tensões;

$\mathrm{t}$ - vetor dos taps dos transformadores;

s - vetor das variáveis de folga ou excesso;

$\lambda$ - vetor dos multiplicadores de Lagrange para as restrições de igualdade;

$\pi$ - vetor dos multiplicadores de Lagrange para as restrições de desigualdade transformada em igualdade pelas variáveis de folga;

$\mu$ - parâmetro de barreira;

$\beta$ - parâmetro utilizado na atualização de $\mu$;

$\mathrm{P}$ - potência ativa;

$\mathrm{P}^{\mathrm{G}}$ - potência ativa gerada;

$\mathrm{P}^{\mathrm{C}}$ - potência ativa consumida;

$\mathrm{P}^{\mathrm{esp}}$ - potência ativa especificada;

$\mathrm{Q}^{\text {esp }}$ - potência reativa especificada;

$P_{i}$ - potência ativa no nó $i$;

Q - potência reativa;

$\mathrm{Q}^{\mathrm{G}}$ - potência reativa gerada;

$\mathrm{Q}^{\mathrm{C}}$ - potência reativa consumida;

$\mathrm{Q}_{\mathrm{j}}$ - potência reativa no nó $\mathrm{j}$;

$\Delta \mathrm{Q}$ - variação de potencia reativa;

$\Delta \mathrm{V}$ - variação de magnitude de tensão;

$\Delta \mathrm{V}_{\max }$ - variação de magnitude de tensão máximo;

I - matriz identidade;

$\nabla \mathrm{L}$ - gradiente da função;

$\Delta$ - correção das variáveis; 
f(x) - função objetivo;

$\mathrm{g}(\mathrm{x})$ - restrições de igualdade;

$\mathrm{h}(\mathrm{x})$ - restrições de desigualdade;

S - função custo;

$\mathrm{K}_{\mathrm{E}}$ - custo de energia;

$\Delta \mathrm{E}$ - variação de energia;

$\mathrm{K}_{\mathrm{p}}$ - custo de potência;

$\Delta \mathrm{P}$ - variação de potência;

$\mathrm{K}_{\mathrm{c}}$ - custo do banco de capacitores;

C - tamanho do banco de capacitores;

x - variáveis de estado ou variáveis de dependentes;

Y - admitância de linha na matriz Y barra;

G - condutância de linha na matriz Y barra;

B - susceptância de linha na matriz Y barra;

y - admitância de linha;

g - condutância da linha;

b - susceptância da linha;

$\Omega$ - conjunto de barras vizinhas a uma barra k;

$\mathrm{K}$ - conjunto das barras vizinhas, incluindo a própria barra k;

k, m - barras terminais de linha;

u - variáveis de controle ou independentes;

$\sigma$ - valor empírico;

$\mathrm{g}_{\mathrm{x}}$ - matriz Jacobiana com relação ao vetor $\mathrm{x}$;

$\mathrm{g}_{\mathrm{u}}$ - matriz Jacobiana com relação ao vetor $\mathrm{u}$; 
SOUSA, T. (2003). Estudo de Planejamento de Reativos em Sistemas Elétricos de Potência. São Carlos, 2003. 71p. Dissertação (Mestrado) - Escola de Engenharia de São Carlos, Universidade de São Paulo.

Este trabalho propõe o desenvolvimento de uma nova abordagem para a resolução do problema de planejamento de reativos em sistemas elétricos de potência. Um levantamento bibliográfico para explicitar as diferentes pesquisas realizadas nesta área foi feito. Este trabalho utiliza os multiplicadores de Lagrange obtidos da solução de um fluxo de potência ótimo, via método dos Pontos Interiores, para determinar as barras onde serão alocados reativos. A partir da determinação destas barras, um problema de programação linear para quantificar a potência reativa a ser alocada, é resolvido. Foram realizados estudos para validação e verificação da abordagem proposta nos sistemas WARD \& HALE de 6 barras, sistema de 8 barras, sistema IEEE 30 e 118 barras.

Palavras-chave: Planejamento Ótimo de Reativos, Fluxo de Potência ótimo, Método dos Pontos Interiores, Sistemas Elétricos de Potência. 
SOUSA, T. (2003). Study of Reactive Planning in Electrical Power Systems. São Carlos, 2003. 71p. Dissertação (Mestrado) - Escola de Engenharia de São Carlos, Universidade de São Paulo.

The present dissertation proposes the development of a novel approach to the solution of reactive planning problem in electrical power systems. A bibliographical survey to show the different researches developed in this area was done. This study uses Lagrange multipliers obtained from the Optimal Power Flow solution, through Interior Point method, to determine the buses where reactive power will be allocated. Starting from the determination of these buses, a Linear Programming problem is solved to quantify the reactive power to be allocated. Studies were carried out for the proposed approach validation and verification in the systems WARD \& HALE of 6 buses, system of 8 buses, systems IEEE of 30 and 118 buses.

Keywords: Optimal Reactive Planning, Optimal Power Flow, Interior Points Method, Electrical Power Systems. 


\section{INTRODUÇÃO}

Com o aumento da demanda nos sistemas elétricos de potência, o gerenciamento de potência reativa tem se tornado cada vez mais importante para as empresas fornecedoras de energia, que necessitam manter as tensões dentro de seus limites pré-estabelecidos, fornecendo, desta forma energia dentro de padrões de qualidade e confiabilidade para os consumidores. Alterações no sistema podem resultar em variações dos níveis de tensões nos barramentos, podendo assim, comprometer a qualidade no fornecimento de energia elétrica. Entretanto, o estado do sistema com níveis de tensões violados pode ser melhorado através dos ajustes dos dispositivos de controle de tensão existentes e caso seja necessário, pelo planejamento ótimo de fontes reativas considerando características físicas, econômicas e de operação.

Trabalhos publicados descrevendo técnicas de solução para o problema de planejamento de reativos são abundantes. Estas técnicas de solução podem ser divididas em quatro categorias: métodos analíticos, métodos de programação 
numérica, métodos heurísticos e métodos baseados em inteligência artificial, ELHAWARY (2002).

$\mathrm{Na}$ prática, muitas empresas fornecedoras de energia elétrica utilizam o conhecimento prático de seus operadores para realizar a alocação de potência reativa. Por sua vez, os operadores realizam seus trabalhos com base em suas experiências com o sistema, fazendo, às vezes, a alocação de reativos de forma não otimizada. Um exemplo disso é considerar que as barras de menor tensão são necessariamente as barras que necessitam de alocação de reativos. Diante da possibilidade de se estar dimensionando de forma errada a localização e a magnitude das fontes reativas a serem instaladas, se faz necessário o uso de um método de determinação destas grandezas com bases científicas, procurando-se chegar o mais próximo do planejamento ótimo destas fontes.

A solução proposta neste trabalho para o planejamento ótimo de reativos, como exemplo de um método de programação numérica, trata a determinação das barras, onde serão alocados os reativos a partir da análise dos multiplicadores de Lagrange, associados às restrições de igualdade de potência reativa e resultantes de um processo de otimização não linear, realizado por um programa de fluxo de potência ótimo. A partir da escolha das barras, é construído um problema de programação linear, que tem como função objetivo minimizar a quantidade de potência reativa a ser instalada nestas barras de forma a trazer o problema de volta a uma solução operacional e a respeitar as restrições do problema de programação linear. 


\section{2) Objetivo}

O objetivo deste trabalho de pesquisa é desenvolver uma nova abordagem para a resolução do problema de alocação de reativos, utilizando o método dos Pontos Interiores para determinar as barras onde serão alocados reativos. A partir da determinação das barras é construído um problema de programação linear com o intuito de quantificar a potência reativa a ser alocada.

\section{3) Organização do Trabalho}

Este capítulo apresenta a justificativa, a motivação e o objetivo para o desenvolvimento deste trabalho e sua organização.

O capítulo 2 apresenta o estado da arte, onde algumas metodologias desenvolvidas para a solução do problema de alocação ótima de reativos são apresentadas de forma resumida.

O capítulo 3 apresenta de forma detalhada o método de Pontos Interiores associado ao problema de fluxo de potência ótimo.

O capítulo 4 apresenta detalhadamente a nova metodologia proposta para a resolução do problema de alocação ótima de reativos.

O capítulo 5 apresenta alguns testes e resultados da abordagem proposta para os sistemas de 6, 8, 30 e 118 barras.

O capítulo 6 apresenta conclusões referentes à abordagem proposta. 


\section{ESTADO DA ARTE DO PROBLEMA DE ALOCAÇÃO DE REATIVOS}

\section{1) Introdução}

Para assegurar a qualidade e a confiabilidade do suprimento de energia para os consumidores é necessário que se mantenha a tensão das barras do sistema entre seus limites pré-estabelecidos. O crescimento de cargas, distante das unidades geradoras; as linhas de transmissão longas, operando em condições de cargas leves; o crescimento dos sistemas de transmissão e sub-transmissão, com a adição de novas linhas podem resultar na variação indesejada da tensão no sistema.

Os efeitos destas mudanças podem ser melhorados pelo operador do sistema através da determinação dos ajustes dos dispositivos de controle de tensão e das fontes reativas existentes no sistema e, caso necessário, da obtenção da expansão ótima de fontes reativas considerando características físicas, econômicas e de operação. O processo de 
alocação de reativos pode ser feito através do ajuste dos "taps" dos transformadores, das tensões dos geradores e também com a alocação de novas fontes de reativos (VAr). Isso possibilita um menor nível de tensão e a minimização das perdas ativa do sistema.

Assim, a alocação de reativos no sistema tem uma dupla função:

- Melhorar os perfis de tensão do sistema, mantendo seus níveis de tensão o mais próximo de seus valores nominais;

- Minimizar as perdas do sistema.

\section{2) Histórico}

Os primeiros trabalhos de alocação ótima de reativos - mais especificamente alocação ótima de capacitores - usaram métodos analíticos. Estes algoritmos foram desenvolvidos quando os recursos computacionais eram caros ou de difícil acesso. Métodos analíticos envolvem o uso de cálculos para determinar o máximo de uma função custo referente à alocação dos capacitores. Essa função custo pode ser dada por:

$$
S=K_{E} \Delta E+K_{P} \Delta P-K_{C} C
$$

Sendo $K_{E} \Delta E$ a economia de energia, $K_{P} \Delta P$ a redução no pico das perdas de potência e $K_{C} C$ o custo de instalação dos capacitores.

Os pioneiros do problema de alocação ótima de capacitores, NEAGLE \& SAMSON (1956), COOK (1959) e, mais tarde, SCHMILL (1965) e BAE (1978), usaram métodos 
analíticos para maximizar algum tipo da função custo dada em (2.1). Embora soluções simples tenham sido alcançadas, estes métodos foram baseados em suposições não realistas de sistemas. Estes primeiros métodos são de fácil entendimento e implementação.

Para encontrar resultados mais exatos, os modelos das linhas foram melhorados. GRAINGER \& LEE (1981) e SALAMA et al. (1985) formularam linhas equivalentes normalizadas, que consideravam linhas com seções de diferentes tamanhos de condutores e cargas distribuídas não uniformemente. GRAINGER \& CIVANLAR (1985) também incluíram o planejamento de capacitores em seus algoritmos e melhoraram seus trabalhos incluindo alocação de reguladores de tensão em publicações subseqüentes. Estes últimos métodos analíticos utilizaram modelamentos reais de linhas de distribuição radiais e consideraram a variação de carga dos sistemas de distribuição.

Uma desvantagem de todos os métodos analíticos é o modelamento dos locais de alocação dos capacitores, e tamanhos destes, como variáveis contínuas. Conseqüentemente, o cálculo do tamanho dos capacitores pode não combinar com os tamanhos padrões disponíveis, e a localização pode não coincidir com a localização física do nó do sistema. Os resultados podem precisar de arredondamentos para um valor acima ou abaixo do valor especificado para se aproximarem do valor prático, podendo acarretar em uma situação de sobretensão ou uma economia menor que a calculada. Os métodos mais recentes são mais exatos e adequados para sistemas de distribuição de tamanhos consideráveis, mas exigem maiores informações dos sistemas e tempo computacional. 
Quando os recursos computacionais tornaram-se mais acessíveis, métodos de programação numérica foram formulados para resolver problemas de otimização. Métodos de programação numérica são técnicas iterativas usadas para maximizar ou minimizar uma função objetivo de variáveis de decisão. Para a alocação ótima de reativos, uma função custo ou uma função perdas podem representar a função objetivo que deve satisfazer a um grupo de restrições. A localização, tamanho ou número de fontes reativas, os valores das tensões e as correntes nas barras podem expressar as variáveis de decisão que devem satisfazer as restrições operacionais. A função objetivo pode considerar todas as tensões e limites de carga na linha, a capacidade das fontes de potência reativa e a localidade física dos nós do sistema. O problema de alocação de fontes reativas no sistema pode ser formulado como segue:

$$
\begin{aligned}
& \text { (MAX) } S=K_{L} \Delta L-K_{C} C \\
& \text { suj à } \Delta V \leq \Delta V_{M A X}
\end{aligned}
$$

Sendo $K_{L} \Delta L$ a economia na redução de energia e na redução das perdas de potência, $K_{C} C$ o custo de instalação e $\Delta V$ a variação de tensão, devido à instalação das fontes de reativos, que não devem exceder um máximo de $\Delta V_{M A X}$.

DURAN (1968) foi o primeiro a usar um método de programação dinâmica para o problema de alocação de capacitores. A formulação é simples e apenas considera a redução da perda de energia e informa o tamanho discreto dos capacitores. Mais tarde, 
FAWZI (1983) seguiu o trabalho de DURAN (1968) incluindo a injeção de reativos às funções de economia.

KISHORE \& HILL (1970) desenvolveram um método para determinar a quantidade mínima de potência reativa necessária para satisfazer as condições das restrições das tensões do sistema. Os autores utilizam uma relação de sensibilidade que mede a relação da magnitude de tensão $V_{i}$ no nó $i$ com a respectiva injeção de potência reativa $Q_{j}$ no nó $j$. A relação linear entre $\Delta V_{i}$ no nó $i$ e o $\Delta Q_{j}$ no nó $j$ é usada na forma de um problema de programação linear, determinando o incremento de potência reativa a ser instalada em vários nós do sistema, fazendo com que a magnitude de tensão fique dentro dos limites desejáveis.

MAMANDUR \& CHENOWETH (1981) desenvolveram uma formulação matemática para o problema de controle ótimo de potência reativa que minimiza as perdas de potência ativa no sistema. O modelo emprega relações linearizadas de sensibilidade do sistema de potência para estabelecer uma relação entre a função objetivo e o desempenho das sensibilidades do sistema relativo as variáveis dependentes e de controle. Foi utilizada uma técnica de programação linear dual para determinar o ajuste ótimo das variáveis de controle, satisfazendo simultaneamente os limites de potência reativa dos geradores; os limites de tensão nas barras de carga e os limites operacionais das variáveis de controle, isto é, a posição dos "taps" dos transformadores, a tensão nos terminais dos geradores e o chaveamento de fontes de potência reativa. Esta técnica é satisfatória para melhorar os perfis de tensão e minimizar as perdas do sistema sob condições operacionais. 
IYER et al. (1984) apresentaram um método de programação numérica de rápida convergência para determinar a magnitude de tensão dos geradores, a posição dos "taps" dos transformadores e a alocação estática de capacitores em um sistema de potência, a fim de minimizar as perdas de potência e melhorar a qualidade de suprimento do sistema. Os valores ótimos para estas variáveis são determinados, definindo-se um problema de otimização sujeito às restrições baseadas nas equações do fluxo de carga e variáveis do sistema. A função objetivo para o problema de otimização representa o rendimento resultante da redução das perdas depois de deduzirem-se as despesas de instalação dos capacitores. As restrições são baseadas nas variáveis do sistema. A função objetivo e as restrições são linearizadas sobre o ponto de operação corrente do sistema decidido por uma programação de geração de potência ativa. A programação usa variável discreta para representar o tamanho dos capacitores e variáveis contínuas para representar a mudança nas tensões dos geradores e "tap's" dos transformadores. As variáveis discretas são transformadas em variáveis binárias. O problema de alocação de capacitores é, então, formulado como um problema de programação linear inteira mista, baseado em decomposição de Benders, em que o problema é decomposto em dois subproblemas menores. As soluções dos dois subproblemas são combinadas para alcançar a solução do problema original. A precisão do modelo foi verificada pelos valores pré-determinados e pelos valores obtidos do fluxo de carga no sistema final.

DEEB \& SHAHIDEHPOUR (1989) apresentaram um método baseado em programação linear para encontrar a solução ótima do problema de alocação de potência reativa. O problema foi decomposto em dois subproblemas - subproblema operacional e subproblema de investimento - baseado no método de decomposição de Benders. O 
objetivo do subproblema operacional é minimizar as perdas de potência ativa e o do subproblema de investimento é minimizar o custo de instalação de novos capacitores e indutores. O método de decomposição Dantzig-Wolfe é implementado para solucionar os subproblemas. Os resultados indicam que o método proposto converge muito rápido com uma solução ótima para problemas operacionais e de investimento.

NOUREDDINE \& CHANDRASEKARAN (1992) linearizaram o problema de minimização de perdas, alocação e tamanho dos capacitores. A linearização é feita em termos de compensação de reativos e mudança de tensão. Esse processo elimina a necessidade de recalcular o problema de fluxo de carga. A fórmula linearizada é derivada das funções de mudanças de tensão e grupos de capacitores. Um exemplo numérico foi considerado para ilustrar as vantagens da técnica de compensação proposta. Uma comparação entre os modelos de programação linear e não linear é apresentada.

GRUDININ (1998) apresentou um modelo de otimização de potência reativa baseado em métodos de programação quadrática sucessiva. A formulação matemática junto ao algoritmo supõe diferentes funções objetivos, dependendo do tipo e propósito do problema. Um modelo de otimização de potência reativa bi-criterioso, que representa o compromisso entre funções objetivos econômicas e de segurança, é proposto. O problema de programação quadrática é resolvido com base no método do tipo Newton. Alguns dos principais problemas em se usar programação quadrática em otimização de potência reativa são: o fato de o processo interagir ciclicamente; a grande dimensão do problema de programação; a complexidade e a confiabilidade dos algoritmos de programação quadrática. 
MANTOVANI et al. (2001b) apresentaram um algoritmo que utiliza a técnica de Decomposição de Benders para resolver o problema de Planejamento de Reativos em Sistemas de Energia Elétrica. A Decomposição de Benders separa o problema de planejamento em dois subproblemas: um subproblema de investimento (mestre) e outro de operação (escravo). O subproblema de operação é resolvido usando-se um algoritmo de programação linear sucessiva (PLS). Já o subproblema de investimento, que é um problema de programação linear inteira (PLI), envolvendo variáveis discretas, é resolvido usando-se um algoritmo do tipo Branch-and-Bound.

REZANIA \& SHAHIDEHPOUR (2001) aplicaram um eficiente método dos pontos interiores preditor-corretor primal-dual para solucionar o problema de otimização de potência reativa através de programação linear sucessiva. Um modelo linear é introduzido, no qual as variáveis de controle e o incremento de tensão são ligados por uma matriz Jacobiana modificada e as perdas na transmissão são representadas como uma função do incremento de tensão. $\mathrm{O}$ método não calcula a inversa da matriz, nem calcula a derivada de segunda ordem da matriz Hessiana a cada iteração, para resolver o programa linear e com isso a solução pode economizar tempo computacional e espaço de memória.

HSIAO \& CHIEN (2001) reformularam o problema de alocação de capacitores como um problema de otimização restrito, multi-objetivo e não diferenciável. Esse trabalho considera quatro diferentes funções objetivos relacionadas ao custo de investimento, à eficiência operacional, à segurança do sistema e à qualidade do serviço. O trabalho emprega um algoritmo de solução de dois estágios, baseado na técnica 
iterativa de restrição $\mathcal{E}$. A noção de um fator de preferência é introduzida através de um processo iterativo para determinar a solução ótima mais satisfatória. Essa formulação não requer que as funções objetivas sejam diferenciáveis e nem que as variáveis sejam contínuas.

ELRAZAZ (2001) propôs um algoritmo para determinar simultaneamente a localização do reator como também seus valores, levando em consideração as diferentes restrições do sistema. $\mathrm{O}$ algoritmo utiliza sensibilidades de potência reativa com respeito às tensões das barras de carga e à compensação de potência reativa para minimizar o custo de instalação dos reatores. Aplicações são usadas para demonstrar a efetividade do algoritmo proposto, em sistemas de potência, operando em condições de carga leve.

CHAVES et al. (2002) apresentaram uma metodologia baseada no uso de ferramentas de otimização e numa hierarquia de tomada de decisões que propiciam a elaboração de planos de expansão dos sistemas de energia. A ferramenta de otimização computacional utilizada para este trabalho foi o PPS (Power System Simulator). O procedimento compreende da entrada de dados, da seleção das barras (candidatas à alocação de reativos), da imposição das restrições do sistema e da escolha dos parâmetros de otimização. No fim, um relatório é apresentado com a solução obtida. Caso a solução não seja satisfatória, são feitas alterações nos parâmetros, seguidas de alterações nas restrições, seguidas de alteração das barras candidatas até que seja alcançada uma solução conveniente. 
Com inspeção a todos os métodos de programação numérica, pode-se observar que o nível de sofisticação e complexidade dos modelos incrementou em ordem cronológica de suas datas de publicação. Vários dos métodos de programação numérica têm a vantagem de considerar a localização das barras nas linhas e o tamanho das fontes reativas como variáveis discretas. Contudo, a preparação dos dados e o desenvolvimento da interface para técnicas numéricas podem exigir um maior tempo computacional que para outros tipos de métodos de programação, como, por exemplo, o método analítico.

Métodos baseados em técnicas de buscas heurísticas, são regras de manuseio desenvolvidas através de intuição, experiência e decisão. Tais métodos produzem estratégias rápidas e práticas, que reduzem o espaço de busca e podem conduzir a uma solução com segurança e que esteja próxima ao ótimo.

ABDEL-SALAM et al. (1994) propuseram uma técnica heurística para identificar uma parte do sistema que possui as maiores perdas devido às correntes de cargas reativas e, então, definiram o ponto sensível na seção com o maior efeito na redução de perdas do sistema. O tamanho dos capacitores colocados nas barras sensíveis é então determinado por maximizar a redução de perdas de potência com a compensação capacitiva. Os métodos heurísticos são intuitivos, de fácil compreensão e simples implementação, se comparados aos métodos analíticos e de programação numérica. Contudo, os resultados produzidos pelos algoritmos heurísticos não garantem bons resultados. 
Outra forma de abordagem é dada pela recente popularidade da inteligência artificial que tem conduzido várias pesquisas a investigarem seu uso para aplicações em engenharia de potência. Em particular, algoritmos genéticos, simulação "annealing", sistemas especialistas, redes neurais artificiais e teoria de lógica "fuzzy" têm sido implementadas no problema de alocação ótima de fontes de reativos, mais especificamente de capacitores e compensadores síncronos.

BOONE \& CHIANG (1993) desenvolveram um método baseado em algoritmos genéticos para determinar o tamanho e a localização ótima das fontes de reativos, mais especificamente dos capacitores. O tamanho e a localização destas fontes são codificados em seqüências binárias, e uma combinação é feita para gerar um novo conjunto de dados capaz de produzir resultados ainda melhores. Esta formulação do problema apenas considera os custos dos capacitores e a redução das perdas de potência do sistema elétrico.

LEE \& YANG (1998) propuseram um estudo comparativo para três algoritmos evolutivos para solução do problema de planejamento ótimo de potência reativa: programação evolutiva, estratégia evolutiva e algoritmos genéticos. Os algoritmos evolutivos são métodos de inteligência artificial para otimização baseados em mecanismos de seleção natural, como mutação, recombinação, reprodução e seleção. Estes métodos compartilham muitas similaridades. Neste trabalho, o problema de planejamento ótimo de potência reativa é decomposto em módulos de otimização $\mathrm{P}$ (potência ativa) e Q (potência reativa) e cada módulo é otimizado pelos algoritmos evolutivos, em um modo iterativo, para obter uma solução global. Os métodos para solução do problema de planejamento ótimo de potência reativa são avaliados pelo 
sistema IEEE-30 barras, e os resultados são comparados com as soluções apresentadas pelos três tipos de algoritmos evolutivos e com a solução apresentada pelo método de programação linear.

MANTOVANI et al. (2001a) propuseram um método que usa a programação linear sucessiva e um algoritmo genético simples para resolver o problema de planejamento ótimo de reativos em sistemas elétricos de potência. O problema é dividido em subproblemas de operação e planejamento. O subproblema operacional, que é um problema não-linear, mal condicionado e não-convexo, consiste na determinação do controle de tensão e do ajuste das fontes reativas. O subproblema de planejamento consiste em obter a expansão ótima das fontes reativas considerando as características operacionais, econômicas e físicas do sistema. A programação linear sucessiva resolve o problema de despacho ótimo de reativos relacionado às variáveis reais, enquanto o algoritmo genético simples é usado para determinar os ajustes necessários entre as variáveis binárias e discretas existentes no problema modelado. O programa implementado dá a localização e tamanho das fontes reativas necessárias, para atender as restrições operacionais e de segurança. O método foi testado na solução do planejamento ótimo de reativos do sistema IEEE-30 barras e de um sistema real de 309 barras. Adotando um tamanho de população conveniente, que é uma função do número de variáveis discretas consideradas pelo algoritmo genético, o algoritmo apresenta uma boa proposta de solução, e o ajuste das variáveis discretas existentes no sistema se dá após a trigésima iteração.

GALLEGO et al. (2001) propuseram um método híbrido originado do método de busca Tabu, estendido com características vindas de outras aproximações 
combinatoriais, como algoritmos genéticos e simulação "annealing”, e de aproximações heurísticas práticas, para solucionar o problema de alocação de reativos em sistemas de distribuição radial. O método foi testado extensivamente em vários sistemas disponíveis na literatura e em sistemas reais, apresentando resultados superiores tanto para soluções de custos como soluções de qualidade.

CHIANG et al. (1990) apresentaram um problema geral de alocação de capacitores, levando em consideração aspectos práticos dos capacitores, limites de cargas e os limites operacionais com diferentes níveis de carga. A nova formulação aproxima a função objetivo custo, não diferenciável, por uma função diferenciável, facilitando a aplicação das técnicas de otimização não lineares. A técnica de simulação "annealing" foi adaptada a problemas de otimização levando-se em consideração certas limitações. Uma das características atrativas do método é a capacidade de busca de um mínimo global da função objetivo, enquanto a maioria das técnicas de otimização estão presas aos mínimos locais. Para a eficiência da metodologia apresentada, foi necessário um eficiente algoritmo de fluxo de carga a fim de conferir a nova configuração gerada pelo método.

SALAMA et al. (1995) desenvolveram um sistema especialista contendo técnicas de habilidade em informação e habilidade humana para o controle de reativos em sistemas de potência. As técnicas de habilidade em informação incluem o método de alocação de capacitores para a máxima redução das perdas de potência e energia. O componente de habilidade humana contém informações para guiar o usuário e para executar o controle 
de potência reativa nas áreas de planejamento, operação e expansão dos sistemas de distribuição.

SANTOSO \& TAN (1990) usaram redes neurais artificiais para o controle ótimo de capacitores. Neste trabalho, duas redes neurais são usadas. Uma rede é usada para predizer o nível de carga de um grupo de valores de carga obtidos da medida direta de várias barras, e uma segunda rede neural é usada para selecionar a posição ótima do "tap" dos capacitores baseada no nível de carga como predito pela primeira rede. A primeira rede é treinada com um grupo de pré-registro de níveis de carga, e a segunda rede é treinada para maximizar a redução de perda energética para uma dada condição de carga. Ambas redes treinadas, cálculos iterativos são exigidos, e uma solução rápida para um dado grupo de entradas pode ser estabelecida. $\mathrm{O}$ algoritmo foi testado em um sistema de 30-barras. Para reduzir a complexidade do treinamento, o caso teste foi separado em seis subsistemas. Embora este método seja satisfatório para uma implementação "on-line" do sistema apresentado, ele pode não ser apropriado para um sistema de distribuição maior. Desde então, pode ser necessário particionar um sistema grande em vários subsistemas menores. E, como é do conhecimento dos próprios autores, o tempo exigido para treinamento da rede neural pode ser muito grande.

ZADEH (1965) introduziu a lógica "fuzzy" como uma ferramenta formal para lidar com um modelamento incerto e flexível. Uma variável "fuzzy" é modelada por um grupo de funções que fixa um grau de agrupamento para o grupo. 
CHIN (1995) usou a teoria de lógica "fuzzy" e fixou três grupos de funções para descrever perdas, divergência de tensão e distorção harmônica. Uma variável de decisão, para determinar os nós onde serão alocadas as fontes de reativos, é então calculado para escolher a interseção dos três grupos de funções para cada nó do sistema. Os nós com melhores valores de decisão são selecionados para instalação das fontes de reativos. Nenhum procedimento de otimização matemática é dado para calcular o tamanho das fontes a serem alocadas nos nós selecionados pela teoria "fuzzy".

H. N. NG et al. (2000a) também aplicaram teoria de lógica "fuzzy" para o problema de alocação de capacitores usando raciocínio aproximado de “fuzzy”. Índices de tensão e perdas de potência dos nós do sistema são modelados por um grupo de funções e um sistema "fuzzy" especialista contendo um grupo de regras heurísticas executa a conclusão para determinar um índice para alocação dos capacitores de cada nó. Os capacitores são alocados aos nós com maior índice de conveniência. O sistema "fuzzy" proposto pode também considerar alguma incerteza nos parâmetros usados ou na falta de dados. 


\section{O PROBLEMA DE FLUXO DE POTÊNCIA ÓTIMO}

\section{1) Introdução}

Um sistema elétrico tem por objetivo fornecer energia dentro de padrões de qualidade e confiabilidade para seus consumidores, para isto, se faz necessário que todas as variáveis do sistema estejam dentro de seus limites previamente especificados. Modificações na configuração da rede, como alterações na geração ou na carga, podem fazer com que as variáveis do sistema sejam alteradas, podendo violar os seus limites. Quando estas variáveis são alteradas, um ajuste nos equipamentos (variáveis de controle) é realizado para situações localizadas. À medida que há um aumento no número de variáveis de controle a serem ajustadas, para satisfazer as condições de operação do sistema, estes ajustes tornam-se um exaustivo processo de tentativas e erros. Neste caso o fluxo de potência ótimo é o instrumento ideal, ajustando simultaneamente, de maneira ótima, todas as variáveis de controle do sistema satisfazendo, critérios pré-estabelecidos.

O problema de fluxo de potência ótimo foi definido no inicio da década de 60. Sua formulação foi inicialmente proposta por CARPENTIER (1962), o qual 
elaborou o problema de fluxo de potência ótimo com base no problema de despacho econômico, que compreende o quanto cada gerador tem de produzir de potência ativa para atender a demanda do sistema. O problema de fluxo de potência ótimo é um problema de programação não linear, não convexo e de grande porte, tornando-se de difícil aplicação em tempo real. Sendo o fluxo de potência ótimo um problema de otimização de grande porte e que, quando resolvido gera um sistema de equações lineares esparsas, qualquer algoritmo eficiente para a solução do problema deve explorar técnicas e estruturas de esparsidade.

Entre as técnicas de programação matemática, utilizadas em algoritmos para solução do fluxo de potência ótimo, podemos citar: programação linear sucessiva (PLS), programação quadrática e métodos de programação não linear baseados em métodos do tipo Newton. Desde a década de 80 , o interesse pela aplicação dos métodos de pontos interiores (MPI), para sistemas de potência, tem aumentado devido ao progresso de seu desempenho e das suas propriedades de convergência.

Neste capítulo será apresentado o método primal-dual barreira logarítmica que será utilizado na determinação das barras onde serão alocadas as fontes reativas.

\section{2) O Problema de Fluxo de Potência Ótimo}

O fluxo de potência ótimo é um problema de otimização restrito e, como já citado anteriormente, não linear, não convexo e de grande porte. A solução deste problema determina o melhor ponto de operação do sistema através da otimização de uma função objetivo que representa um dado desempenho do sistema, como exemplo, a minimização das perdas ativas na transmissão, a minimização dos custos de geração, etc. O fluxo de potência ótimo pode ser representado matematicamente 
através de um problema geral de otimização com restrições de igualdade e desigualdade como:

$$
\begin{array}{ll}
\text { Min } & f(x) \\
\text { s.a.: } & g(x)=0 \\
& \underline{h} \leq h(x) \leq \bar{h} \\
& \underline{x} \leq x \leq \bar{x}
\end{array}
$$

O vetor das variáveis de estado $x$ representa a magnitude de tensão $(V)$, ângulo $(\theta)$ e tap dos transformadores $(t)$. A função objetivo $f(x)$ é uma função escalar e representa, para os nossos estudos, as perdas de potência ativa na transmissão. Essa função é não separável e não permite simplificações.

As restrições de igualdade $g(x)$ são as equações do fluxo de potência obtidas quando impõe-se o princípio da conservação de potência em cada barra da rede. As restrições de desigualdade $h(x)$ representam as restrições funcionais, como a potência reativa nas barras de controle de reativos, os fluxos ativos e reativos nas linhas de transmissão, fluxo de intercâmbio, etc.

Ao rescrever (3.1) utilizando as equações de fluxo de potência apresentadas por MONTICELLI (1983), temos o seguinte problema de fluxo de potência ótimo: 


$$
\begin{array}{lll}
\text { Min } & \sum_{i=1}^{N L} g_{i}\left[V_{k}^{2}+V_{m}^{2}-2 V_{k} V_{m} \cos \theta_{k m}\right] & \\
\text { s.a.: } & P_{k}^{G}-P_{k}^{C}-V_{k} \sum_{m \in k} V_{m}\left(G_{k m} \cos \theta_{k m}+B_{k m} \operatorname{sen} \theta_{k m}\right)=0 & k=1, \ldots, N B C C R \\
& Q_{k}^{G}-Q_{k}^{C}-V_{k} \sum_{m \in k} V_{m}\left(G_{k m} \operatorname{sen} \theta_{k m}-B_{k m} \cos \theta_{k m}\right)=0 & k=1, \ldots, N B C \\
\underline{Q}_{j} \leq V_{j} \sum_{m \in k} V_{m}\left(G_{k m} \operatorname{sen} \theta_{k m}-B_{k m} \cos \theta_{k m}\right) \leq \bar{Q}_{j} & j=1, \ldots, N B C R \\
\underline{t}_{i} \leq t \leq \bar{t}_{i} & i=1, \ldots, N T \\
\underline{V}_{k} \leq V_{k} \leq \bar{V}_{K} & k=1, \ldots, N B
\end{array}
$$

O problema de fluxo de potência ótimo com função objetivo representando as perdas ativas na transmissão é conhecido como fluxo de potência ótimo reativo. Essa definição se dá pelo fato de todas as variáveis associadas às potências ativas estarem fixas, com exceção da potência gerada na barra de referência. A função objetivo, apresentada pela equação (3.2), representa as perdas ativas nas linhas de transmissão, sendo considerada um fator complicante para a solução do problema de fluxo de potência ótimo, por ser uma função não linear.

Muitas técnicas de otimização para a solução do problema de fluxo de potência ótimo foram estudadas. A técnica de solução que utiliza pontos interiores tem se mostrado uma alternativa eficiente na solução de problemas de otimização de sistemas de potência. Entre os métodos de otimização que utilizam pontos interiores, o método primal-dual barreira logarítmica tem sido aplicado para solução do problema de fluxo de potência ótimo, GRANVILLE (1994). 


\section{3) O Método Primal-Dual Barreira Logarítmica associado ao problema de Fluxo de Potência Ótimo}

A seguir será apresentado o método primal-dual barreira logarítmica (PDBL), SOUSA (2001). Para tal apresentação, e ainda para facilitar a notação, será usado o problema de fluxo potência ótimo apresentado pela equação (3.1). Para a resolução deste problema pelo método PDBL, é necessário que as restrições de desigualdades se tornem igualdades através da adição de variáveis de folga ou excesso, positivas. O problema modificado pode ser rescrito como:

$$
\begin{array}{ll}
\text { Min } & f(x) \\
\text { s.a.: } & g(x)=0 \\
& h(x)+s_{1}=\bar{h} \\
& h(x)-s_{2}=\underline{h} \\
& x+s_{3}=\bar{x} \\
& x-s_{4}=\underline{x} \\
& s_{1}, s_{2}, s_{3}, s_{4} \geq 0
\end{array}
$$

Estas variáveis de folga e excesso estritamente positivas são incorporadas à função objetivo através da função barreira logarítmica. Assim, o problema (3.3) passa a ser rescrito da seguinte forma:

$$
\begin{array}{ll}
\operatorname{Min}\{ & \left.f(x)-\mu \sum_{i=1}^{N B C R} \ln \left(s_{1 i}\right)-\mu \sum_{i=1}^{N B C R} \ln \left(s_{2 i}\right)-\mu \sum_{i=1}^{N B} \ln \left(s_{3 i}\right)-\mu \sum_{i=1}^{N B} \ln \left(s_{4 i}\right)\right\} \\
\text { s.a.: } \quad & g(x)=0 \\
& h(x)+s_{1}=\bar{h} \\
& h(x)-s_{2}=\underline{h} \\
& x+s_{3}=\bar{x} \\
& x-s_{4}=\underline{x}
\end{array}
$$

Sendo $\mu$ o parâmetro de barreira, positivo, que tende a zero durante o processo de otimização, isto é, $\mu_{0}>\mu_{1}>\mu_{2}>\ldots>\mu_{\infty}=0$. 
Os termos logarítmicos asseguram que as condições das variáveis de folga ou excesso serão estritamente positivas. A partir do problema restrito, representado por (3.4), constrõe-se a função Lagrangiana, como mostrado a seguir:

$$
\begin{aligned}
L= & f(x)-\mu \sum_{i=1}^{N B C R} \ln \left(s_{1 i}\right)-\mu \sum_{i=1}^{N B C R} \ln \left(s_{2 i}\right)-\mu \sum_{i=1}^{N B} \ln \left(s_{3 i}\right)-\mu \sum_{i=1}^{N B} \ln \left(s_{4 i}\right)-\lambda^{t} g(x) \\
& -\pi_{1}\left(h(x)+s_{1}-\bar{h}\right)-\pi_{2}\left(h(x)-s_{2}-\underline{h}\right)-\pi_{3}\left(x+s_{3}-\bar{x}\right)-\pi_{4}\left(x-s_{4}-\underline{x}\right)
\end{aligned}
$$

Sendo $\lambda, \pi_{1}, \pi_{2}, \pi_{3}$ e $\pi_{4}$ vetores dos multiplicadores de Lagrange.

Os multiplicadores de Lagrange, $\lambda_{i}$ representam a mudança marginal do valor ótimo na função objetivo enquanto a disponibilidade da quantidade de recurso i disponível está sendo variada. Em outras palavras, $\lambda_{i}$, mostra o acréscimo ou decréscimo na função objetivo, por acréscimo unitário na disponibilidade de recurso $i$, FRITZSCHE (1978). Isso pode ser representado por:

$$
\frac{\partial f(x(b))}{\partial b_{i}}=\lambda_{i}
$$

Sendo $x(b)$ o valor ótimo da função e $b_{i}$ uma limitação da quantidade de recurso $i$ disponível.

Aplicando as condições de otimalidade em (3.5), obtém-se o seguinte sistema de equações: 
$\nabla_{x} L=\nabla_{x} f(x)-\nabla_{x} g(x)^{t} \lambda-\nabla_{x} h(x)^{t} \pi_{1}-\nabla_{x} h(x)^{t} \pi_{2}-\pi_{3}-\pi_{4}$

$\nabla_{\lambda} L=-g(x)=0$

$\nabla_{\pi_{i}} L=-\left(h(x)+s_{1}-\bar{h}\right)=0$

$\nabla_{\pi_{2}} L=-\left(h(x)-s_{2}-\underline{h}\right)=0$

$\nabla_{\pi_{3}} L=-\left(x+s_{3}-\bar{x}\right)=0$

$\nabla_{\pi_{4}} L=-\left(x-s_{4}-\underline{x}\right)=0$

$\nabla_{s_{1}} L=-\mu S_{1}^{-1} e-\pi_{1}=0$

$\nabla_{s_{2}} L=-\mu S_{2}^{-1} e+\pi_{2}=0$

$\nabla_{s_{3}} L=-\mu S_{3}^{-1} e-\pi_{3}=0$

$\nabla_{s_{4}} L=-\mu S_{4}^{-1} e+\pi_{4}=0$

Sendo $e=(1,1, \ldots, 1)^{t}, S_{1}, S_{2}, S_{3}, S_{4}$ matrizes diagonais, cujos elementos são $s_{1}, s_{2}, s_{3}, s_{4}$, respectivamente e $\nabla f(x)$ é o gradiente de $f(x)$.

\subsection{1) O Método de Newton}

O método de Newton é utilizado com o intuito de solucionar o sistema de equações (3.7) a (3.16). Este método utiliza a expansão em série de Taylor até primeira ordem das equações do sistema, e gera as direções de busca $\left(\Delta x, \Delta \lambda, \Delta \pi_{1}, \Delta \pi_{2}, \Delta \pi_{3}, \Delta \pi_{4}, \Delta s_{1}, \Delta s_{2}, \Delta s_{3}, \Delta s_{4}\right)$ que serão utilizadas para a atualização das variáveis do sistema. Desta forma, as equações de Newton para se obterem as direções são as seguintes:

$$
\begin{aligned}
& W\left(x, \lambda, \pi_{1}, \pi_{2}\right) \Delta x-\nabla_{x} g(x)^{t} \Delta \lambda-\nabla_{x} h(x)^{t} \Delta \pi_{1}-\nabla_{x} h(x)^{t} \Delta \pi_{2}-\Delta \pi_{3}-\Delta \pi_{4}=-\nabla_{x} L \\
& -\left(\nabla_{x} g(x)\right) \Delta x=-\nabla_{\lambda} L \\
& -\left(\nabla_{x} h(x) \Delta x+\Delta s_{1}\right)=-\nabla_{\pi_{i}} L \\
& -\left(\nabla_{x} h(x) \Delta x-\Delta s_{2}\right)=-\nabla_{\pi_{2}} L \\
& -\left(\Delta x+\Delta s_{3}\right)=-\nabla_{\pi_{3}} L \\
& -\left(\Delta x-\Delta s_{4}\right)=-\nabla_{\pi_{4}} L \\
& \mu S_{1}^{-2} \Delta s_{1}-\Delta \pi_{1}=-\nabla_{s_{1}} L \\
& \mu S_{2}^{-2} \Delta s_{2}+\Delta \pi_{2}=-\nabla_{s_{2}} L \\
& \mu S_{3}^{-2} \Delta s_{3}-\Delta \pi_{3}=-\nabla_{s_{3}} L \\
& \mu S_{4}^{-2} \Delta s_{4}+\Delta \pi_{4}=-\nabla_{s_{4}} L
\end{aligned}
$$


Rescrevendo (3.17) na forma matricial, tem-se:

$\left[\begin{array}{cccccccccc}W\left(x, \lambda, \pi_{1}, \pi_{2}\right) & -J(x)^{t} & -\nabla_{x} h(x)^{t} & -\nabla_{x} h(x)^{t} & -I & -I & 0 & 0 & 0 & 0 \\ -J(x) & 0 & 0 & 0 & 0 & 0 & 0 & 0 & 0 & 0 \\ -\nabla_{x} h(x) & 0 & 0 & 0 & 0 & 0 & -I & 0 & 0 & 0 \\ -\nabla_{x} h(x) & 0 & 0 & 0 & 0 & 0 & 0 & I & 0 & 0 \\ -I & 0 & 0 & 0 & 0 & 0 & 0 & 0 & -I & 0 \\ -I & 0 & 0 & 0 & 0 & 0 & 0 & 0 & 0 & I \\ 0 & 0 & -I & 0 & 0 & 0 & \mu S_{I}^{-2} & 0 & 0 & 0 \\ 0 & 0 & 0 & I & 0 & 0 & 0 & \mu S_{2}^{-2} & 0 & 0 \\ 0 & 0 & 0 & 0 & -I & 0 & 0 & 0 & \mu S_{3}^{-2} & 0 \\ 0 & 0 & 0 & 0 & 0 & I & 0 & 0 & 0 & \mu S_{4}^{-2}\end{array}\right]\left[\begin{array}{c}\Delta x \\ \Delta \lambda \\ \Delta \pi_{1} \\ \Delta \pi_{2} \\ \Delta \pi_{3} \\ \Delta \pi_{4} \\ \Delta s_{1} \\ \Delta s_{2} \\ \Delta s_{3} \\ \Delta s_{4}\end{array}\right]=-\left[\begin{array}{c}\nabla_{x} L \\ \nabla_{\lambda} L \\ \nabla_{\pi_{i}} L \\ \nabla_{\pi_{2}} L \\ \nabla_{\pi_{s}} L \\ \nabla_{\pi_{s}} L \\ \nabla_{s_{i}} L \\ \nabla_{s_{2}} L \\ \nabla_{s_{3}} L \\ \nabla_{s_{s}} L\end{array}\right] \quad(3.18)$

Sendo:

$W\left(x, \lambda, \pi_{1}, \pi_{2}\right)=\nabla^{2} f(x)-\sum_{i=l}^{2 x N B} \lambda_{I} \nabla^{2} g_{i}(x)-\sum_{i=1}^{N B C R} \pi_{l i} \nabla^{2} h(x)-\sum_{i=l}^{N B C R} \pi_{2 i} \nabla^{2} h(x) ;$

$J(x)=\nabla_{x} g(x)$, a matriz Jacobiana do fluxo de carga convencional;

$I$, a matriz identidade.

Pode-se escrever $W\left(x, \lambda, \pi_{1}, \pi_{2}\right)$ na forma matricial:

$$
W=\left[\begin{array}{lll}
\frac{\partial^{2} L}{\partial t^{2}} & \frac{\partial^{2} L}{\partial t \partial \theta} & \frac{\partial^{2} L}{\partial t \partial V} \\
\frac{\partial^{2} L}{\partial \theta \partial t} & \frac{\partial^{2} L}{\partial \theta^{2}} & \frac{\partial^{2} L}{\partial \theta \partial V} \\
\frac{\partial^{2} L}{\partial V \partial t} & \frac{\partial^{2} L}{\partial V \partial \theta} & \frac{\partial^{2} L}{\partial V^{2}}
\end{array}\right]
$$

O sistema matricial (3.18) pode ser representado da seguinte maneira:

$$
L a * \Delta d=-\nabla L
$$

Sendo:

$L a=$ matriz Lagrangiana;

$\Delta d=$ vetor das direções de busca;

$\nabla L=$ vetor gradiente 


\subsection{2) Matriz Lagrangiana}

A matriz Lagrangiana ( $L a$ ), do sistema (3.18), é esparsa, ou seja, possui muitos elementos nulos, e esta característica deve ser explorada no processo de solução do sistema. A dimensão da matriz Lagrangiana $(L a)$, construída devido à aplicação do método primal-dual barreira logarítmica para a solução do problema (3.1) é maior que a dimensão da matriz utilizada nos métodos da Penalidade, de Newton, entre outros. Nestes métodos o sistema a ser resolvido é apenas uma parte do sistema dado em (3.18), isto é:

$$
\left.\left[\begin{array}{cc}
W(x, \lambda) & -J(X) \\
-J(X) & 0
\end{array}\right] \begin{array}{c}
\Delta x \\
\Delta \lambda
\end{array}\right]=-\left[\begin{array}{c}
\nabla_{x} L \\
\nabla_{\lambda} L
\end{array}\right]
$$

A matriz Lagrangiana em (3.18) tem dimensão total $(\mathrm{N})$ dada por:

$$
N=8 * N B+3 * N B C R+5 * N T-3
$$

Esta matriz possui elementos unitários e as submatrizes referentes às variáveis de folga são de fácil implementação. Assim, o esforço computacional necessário para a obtenção de (3.18) não é muito elevado, quando comparado com os métodos que utilizam a expressão (3.21).

\subsection{3) Tamanho do Passo e Atualização das Variáveis}

Conhecendo as direções de busca, a próxima etapa é calcular os passos $\alpha_{p} \mathrm{e}$ $\alpha_{d}$, que serão usados na atualização das variáveis primais e duais respectivamente. Estes passos são calculados de maneira que cada componente das variáveis de folga ou excesso primais $\left(s_{i}, i=1,2,3,4\right)$ permaneçam estritamente positivas, e que os 
elementos do vetor $\left(\pi_{i}, i=1,2,3,4\right)$ permaneçam com os seus respectivos sinais, isto é, $\pi_{1}<0, \pi_{2}>0, \pi_{3}<0, \pi_{4}>0$.

Esse processo é feito encontrando-se o menor elemento entre o mínimo tamanho de passo de todos os componentes dos vetores. Em seguida este menor elemento é multiplicado por um fator $\sigma$, menor que "1", que garante que o próximo ponto irá satisfazer as condições de positividade e, então, comparado com o valor "1", o menor entre eles é escolhido como tamanho do passo primal ou dual.

Isso se traduz por:

$$
\begin{aligned}
& \alpha_{p}=\min \left\{\sigma\left(\min _{\Delta S_{1}<0} \frac{s_{1}}{\left|\Delta S_{1}\right|}, \min _{\Delta S_{2}<0} \frac{s_{2}}{\left|\Delta S_{2}\right|}, \min _{\Delta S_{3}<0} \frac{s_{3}}{\left|\Delta S_{3}\right|}, \min _{\Delta S_{4}<0} \frac{s_{4}}{\left|\Delta S_{4}\right|}\right), 1\right\} \\
& \alpha_{d}=\min \left\{\sigma\left(\min _{\Delta \pi_{1}>0} \frac{-\pi_{1}}{\left|\Delta \pi_{l}\right|}, \min _{\Delta \pi_{2}<0} \frac{\pi_{2}}{\left|\Delta \pi_{2}\right|}, \min _{\Delta \pi_{3}>0} \frac{-\pi_{3}}{\left|\Delta \pi_{3}\right|}, \min _{\Delta \pi_{+}<0} \frac{\pi_{4}}{\left|\Delta \pi_{4}\right|}\right), 1\right\}
\end{aligned}
$$

Sendo $\sigma=0,9995$ um valor determinado empiricamente.

Conhecendo as direções de busca e os passos primais e duais respectivamente, todas as variáveis do problema podem ser atualizadas por:

$$
\begin{array}{lc}
x=x+\alpha_{p} \Delta x & \lambda=\lambda+\alpha_{d} \Delta \lambda \\
s_{1}=s_{1}+\alpha_{p} \Delta s_{1} & \pi_{1}=\pi_{1}+\alpha_{d} \Delta \pi_{1} \\
s_{2}=s_{2}+\alpha_{p} \Delta s_{2} & \pi_{2}=\pi_{2}+\alpha_{d} \Delta \pi_{2} \\
s_{3}=s_{3}+\alpha_{p} \Delta s_{3} & \pi_{3}=\pi_{3}+\alpha_{d} \Delta \pi_{3} \\
s_{4}=s_{4}+\alpha_{p} \Delta s_{4} & \pi_{4}=\pi_{4}+\alpha_{d} \Delta \pi_{4}
\end{array}
$$




\subsection{4) O Parâmetro de Barreira}

Uma etapa muito importante no algoritmo primal-dual barreira logarítmica é a escolha inicial do parâmetro de barreira. $\mathrm{O}$ valor do multiplicador $\mu$ para cada ponto é proporcional ao gap de dualidade. Este gap é a diferença entre o valor da função objetivo do problema primal e o valor da função objetivo do problema dual. A cada iteração o valor de $\mu$ deverá ser calculado de tal forma que o seu valor atual seja sempre inferior ao anterior e, desta forma, é proposto um cálculo para atualização de $\mu$, utilizando a equação (3.26), na qual o numerador corresponde ao gap de dualidade:

$$
\mu=\frac{-\left(s_{1} \pi_{1}+s_{3} \pi_{3}\right)+\left(s_{2} \pi_{2}+s_{4} \pi_{4}\right)}{2 * N B * \beta}
$$

Sendo $\beta>1$ e especificado pelo usuário.

Como visto em (3.26) o parâmetro $\mu$ é reduzido a cada iteração de forma empírica, pois o valor de $\beta$ é determinado pelo usuário. Uma escolha inadequada do parâmetro $\beta$ poderá comprometer a convergência do método. Outra forma para atualizar o parâmetro $\mu$ é reduzi-lo a cada iteração de um valor $\beta$ ' (Granville, 1994), especificado pelo usuário, isto é:

$$
\mu^{(k+1)}=\frac{\mu^{k}}{\beta}
$$




\subsection{5) Inicialização das Variáveis}

Com respeito aos valores iniciais das variáveis, a única exigência que deve ser observada é que as variáveis do sistema, ou seja, as tensões, os taps e as injeções de reativos devem estar dentro de seus limites pré-estabelecidos. As equações de balanço do sistema e as inequações, que foram transformadas em equações através da inclusão das variáveis de folga ou excesso, não precisam ser satisfeitas na inicialização do problema. As variáveis de folga ou excesso $\left(s_{i}, i=1,2,3,4\right)$ podem ser inicializadas utilizando as equações (3.9) a (3.12) respectivamente.

Estas variáveis devem ser estritamente positivas. Isto é esperado desde que as variáveis do sistema estejam dentro de seus limites, porém, caso exista algum componente deste vetor, que seja nulo ou negativo, o mesmo poderá assumir o valor 0,02 como proposto por QUINTANA et al. (1995). Após terem sido inicializados os vetores referentes às variáveis de folga, os vetores dos multiplicadores de Lagrange $\left(\pi_{i}, i=1,2,3,4\right)$ podem ser inicializados utilizando-se as equações (3.13) a (3.16), respectivamente. $\mathrm{E}$ os vetores dos multiplicadores de Lagrange para as restrições de igualdades $(\lambda)$ são sempre inicializados em zero. $O$ valor inicial do parâmetro de barreira e o multiplicador $\beta$ para atualização de $\mu$ são determinados pelo usuário, como mencionado anteriormente. 


\section{FORMULAÇÃo DO PROBLEMA DE PLANEJAMENTO DE REATIVOS EM SISTEMAS ELÉTRICOS DE POTÊNCIA}

\section{1) Introdução}

O problema do planejamento ótimo de reativos para sistemas elétricos de potência pode ser formulado como um problema de alocação de fontes reativas em um sistema de energia elétrica, a partir de cenários de operação definidos considerando restrições de segurança e de atendimento da demanda. As características econômicas, físicas e de operação das fontes de reativos existentes, e das novas fontes a serem instaladas no sistema, devem estar bem definidas para a obtenção de uma proposta adequada de planejamento.

$\mathrm{O}$ conjunto dos equipamentos que constitui um sistema de energia elétrica geralmente é capaz de suprir toda a demanda ativa e reativa para manter o sistema em condições normais de carga e de topologia. A demanda reativa necessária é suprida pelas capacidades de geração de reativos das máquinas geradoras, pelas linhas de transmissão longas, pela eliminação de problemas de violações de tensões 
através do uso de transformadores com taps variáveis e por uma topologia adequada do sistema.

O sistema planejado considerando certas condições de carga e topologia tem reservas reativas necessárias para operar atendendo às restrições de segurança e de operação. Com a evolução do sistema, o sistema originalmente planejado sofre alterações de topologia e de crescimento de cargas podendo não mais atender as restrições do sistema. Assim, as principais condições que exigem o planejamento de reativos são crescimento de cargas distantes das unidades geradoras, causando problemas na qualidade do serviço; problemas de instabilidade de tensão e perdas excessivas na transmissão; linhas de transmissão longas de alta tensão operando em condições de cargas leves, o que causa problemas de sobretensões nos terminais; necessidade de uma estratégia adequada de planejamento; crescimento dos sistemas de transmissão e subtransmissão, e necessidade de substituir equipamentos por motivos técnicos e/ou econômicos.

O planejamento ótimo de reativos consiste em determinar, considerando todos os cenários definidos, a localização e a magnitude das fontes reativas que assegurem a operação do sistema de energia elétrica atendendo os limites físicos e de segurança pré-estabelecidos, MANTOVANI (1994).

Na formulação e solução do problema deve-se considerar alguns aspectos importantes: as fontes reativas e os controles disponíveis precisam ser levados em consideração na alocação de novas fontes, e o conjunto de barras à alocação de novas fontes deve ser também um processo de otimização incorporado ao problema do planejamento ótimo de reativos. 


\section{2) Escolha das Barras para a Alocação de Reativos}

Um item importante no problema de alocação de reativos é a escolha correta das barras para a alocação de reativos. É necessário que esta escolha tenha um conteúdo científico incorporado ao problema de alocação de reativos. Na literatura especializada as barras são escolhidas pelos operadores do sistema, usando muitas das vezes métodos de escolha heurísticos.

O modelo proposto neste trabalho para a solução do planejamento de reativos trata a determinação destas barras a partir de uma análise aos multiplicadores de Lagrange associados às restrições de igualdade de potência reativa, resultantes de um programa de fluxo de potência ótimo.

A partir do ponto de operação inicial do sistema, um programa de fluxo carga convencional é executado. Caso a resposta do fluxo de carga convencional esteja fora de seus limites será feita uma análise aos multiplicadores de Lagrange associados às restrições de igualdade de potência reativa. Observa-se, então, qual destes multiplicadores tem maior sensibilidade em relação à variação de potência reativa. Assim, as barras do sistema referentes a esses multiplicadores tornam-se as barras onde serão alocados os reativos. 


\section{3) O Problema de Fluxo de Carga}

A seguir será apresentada a formulação do problema de fluxo de carga que terá parte de sua matriz Jacobiana, resultante do processo de linearização das equações não lineares, utilizada na determinação da matriz sensibilidade $S$.

Considere-se que a modelagem do sistema elétrico de potência é representada por um conjunto de equações e inequações algébricas. Assim, a solução do problema de fluxo de carga tem como objetivo determinar o ponto de operação do sistema de energia elétrica, ou seja, as tensões e os ângulos em todas as barras, e os taps dos transformadores. Estas equações são obtidas impondo-se o princípio da conservação das potências ativas e reativas em cada barra do sistema, enquanto que as inequações são dadas pelas restrições das magnitudes de tensões, pelos limites das injeções de potência reativa nas barras de controle de reativo e pelos taps de transformadores.

As equações de fluxo de carga para um sistema elétrico de potência são dadas por (MONTICELLI, 1983):

$$
\begin{aligned}
& \Delta P_{K}=P_{K}^{e s p}-V_{K}^{2} G_{K K}-V_{K} \sum_{m \in \Omega} V_{m}\left(G_{K m} \cos \theta_{K m}+B_{K m} \operatorname{sen} \theta_{K m}\right)=0 \\
& \Delta Q_{K}=Q_{K}^{e s p}+Q_{K}^{s h}+V_{K}^{2} B_{K K}-V_{K} \sum_{m \in \Omega} V_{m}\left(G_{K m} \operatorname{sen} \theta_{K m}-B_{K m} \cos \theta_{K m}\right)=0
\end{aligned}
$$

Para $k=1, \ldots, N B$ e:

$$
\begin{aligned}
& P_{k}^{e s p}=P_{k}^{G}-P_{k}^{c} \\
& Q_{k}^{e s p}=Q_{k}^{G}-Q_{k}^{c}
\end{aligned}
$$


As inequações do fluxo de carga são dadas por:

$$
\begin{aligned}
& V_{k}^{\text {min }} \leq V_{k} \leq V_{k}^{\text {max }} \\
& Q_{k}^{\text {min }} \leq Q_{k} \leq Q_{k}^{\text {max }} \\
& t_{i}^{\text {min }} \leq t_{i} \leq t_{i}^{\text {max }}
\end{aligned}
$$

Sendo $t_{i} \mathrm{o}$ tap do transformador da linha $i$.

Um dos métodos utilizados para a solução do problema é o método de Newton-Raphson, que consiste na expansão, em série de Taylor até $1^{\mathrm{a}}$ ordem, das equações. O sistema linearizado (4.4) é resolvido iterativamente, até que o critério de convergência estabelecido seja atingido (MONTICELLI, 1983).

$$
g(x)=J(x) \Delta x
$$

Onde $g(x)$ representa o conjunto de equações:

$$
g(x)=\left[\begin{array}{l}
\Delta P(x) \\
\Delta Q(x)
\end{array}\right]=0
$$

e:

$x=$ vetor das variáveis dependentes, $x=\left[\begin{array}{c}\theta \\ V \\ t\end{array}\right]$;

$\Delta x=$ vetor das correções;

$J(x)=$ matriz Jacobiana.

A matriz Jacobiana é dada por:

$$
J=\left[\begin{array}{ll}
H & N \\
M & L
\end{array}\right]
$$

Sendo as componentes das submatrizes jacobianas $H, N, M, L$ dadas por: 
$\mathrm{H}\left\{\begin{array}{l}H_{k m}=\partial P_{k} / \partial \theta_{m}=V_{k} V_{m}\left(G_{k m} \operatorname{sen} \theta_{k m}-B_{k m} \cos \theta_{k m}\right) \\ H_{k k}=\partial P_{k} / \partial \theta_{k}=-V_{k}^{2} B_{k k}-V_{k} \sum_{m \in K} V_{m}\left(G_{k m} \operatorname{sen} \theta_{k m}-B_{k m} \cos \theta_{k m}\right)\end{array}\right.$

$N\left\{\begin{array}{l}N_{k m}=\partial P_{k} / \partial V_{m}=V_{k}\left(G_{k m} \cos \theta_{k m}+B_{k m} \operatorname{sen} \theta_{k m}\right) \\ N_{k k}=\partial P_{k} / \partial V_{k}=V_{k} G_{k k}+\sum_{m \in K} V_{m}\left(G_{k m} \cos \theta_{k m}+B_{k m} \operatorname{sen} \theta_{k m}\right)\end{array}\right.$

$M\left\{\begin{array}{l}M_{k m}=\partial Q_{k} / \partial \theta_{m}=-V_{k} V_{m}\left(G_{k m} \cos \theta_{k m}+B_{k m} \operatorname{sen} \theta_{k m}\right) \\ M_{k k}=\partial Q_{k} / \partial \theta_{k}=-V_{k}^{2} G_{k k}+V_{k} \sum_{m \in K} V_{m}\left(G_{k m} \cos \theta_{k m}+B_{k m} \operatorname{sen} \theta_{k m}\right)\end{array}\right.$

$L\left\{\begin{array}{l}L_{k m}=\partial Q_{k} / \partial V_{m}=V_{k}\left(G_{k m} \operatorname{sen} \theta_{k m}-B_{k m} \cos \theta_{k m}\right) \\ L_{k k}=\partial Q_{k} / \partial V_{k}=-V_{k} B_{k k}+\sum_{m \in K} V_{m}\left(G_{k m} \operatorname{sen} \theta_{k m}-B_{k m} \cos \theta_{k m}\right)\end{array}\right.$

Determinado o ponto de operação do sistema, $x=x_{0}$, verifica-se se as tensões estão ou não dentro de seus limites. Caso não estejam, há a necessidade de injetar reativos no sistema, através de equipamentos existentes ou, na falta destes, alocar novas fontes de reativos.

\section{4) Obtenção da Matriz de Sensibilidade}

A matriz sensibilidade entre tensão e potência reativa será utilizada na construção do problema de programação linear, na alocação de reativos. 
Considere um vetor $u$, formado pelas variáveis independentes ou controle do sistema, que, neste problema, corresponde às injeções de reativos $\left(Q_{k}^{s h}\right)$ a serem instaladas em $m$ barras do sistema. Como mencionado anteriormente, caso $x=x_{0}$ seja solução do problema de fluxo de carga para um dado vetor $u=u_{0}$ especificado, temse que:

$$
g\left(x_{0}, u_{0}\right)=0
$$

Suponha que uma pequena variação $\Delta u$ no vetor $u$ cause uma mudança $\Delta x$ no vetor $x$, então a expansão em série de Taylor da equação (4.10) até $1^{\mathrm{a}}$ ordem é dada por:

$$
g\left(x_{0}+\Delta x, u_{0}+\Delta u\right)=g\left(x_{0}, u_{0}\right)+g_{x} \Delta x+g_{u} \Delta u=0
$$

Sendo $g_{x}$ a matriz Jacobiana de $g(x, u)$ com relação ao vetor $x$. A matriz $g_{x}$ é quadrada e não singular, sendo definida por:

$$
g_{x}=\frac{\partial\left(g_{1}, g_{2}, \ldots, g_{2 n b}\right)}{\partial\left(x_{1}, x_{2}, \ldots, x_{2 n b}\right)}
$$

Sendo $g_{u}$ a matriz Jacobiana de $g(x, u)$ com relação ao vetor $u$. A matriz $g_{u}$ não é necessariamente quadrada, sendo definida por:

$$
g_{u}=\frac{\partial\left(g_{1}, g_{2}, \ldots, g_{2 n b}\right)}{\partial\left(u_{1}, u_{2}, \ldots, u_{m}\right)}
$$

Sendo $\Delta x \mathrm{e} \quad \Delta u$ os vetores de correções de $x$ e $u$, respectivamente. Combinando as equações (4.10) e (4.11) tem-se que:

$$
g_{x} \Delta x+g_{u} \Delta_{u}=0
$$


Rescrevendo a equação acima, pode-se definir a matriz de sensibilidade $[S]$ como sendo:

$$
S=\frac{\Delta x}{\Delta u}=-g_{x}^{-1} * g_{u}
$$

Para um caso geral a dimensão da matriz de sensibilidade $[S]$ é igual a $2 n b \mathrm{x}$ $m$, sendo $m$ o número de variáveis independentes.

\section{5) Formulação do Problema de Programação Linear}

O vetor $x$, como mencionado anteriormente, é formado pelas variáveis dependentes do sistema, ou seja, pelas tensões e seus respectivos ângulos. Com o intuito de alocar reativos no sistema, apenas parte deste vetor será utilizada, isto é, as magnitudes das tensões nas barras de carga. Assim, considerando que uma mudança na injeção de reativo $\Delta Q_{j}$ na barra $j$, cause uma variação $\Delta V_{i}$ na tensão da barra de carga $i$, a equação (4.15) na forma matricial, em função das variáveis do problema, (KISHORE, 1971), pode ser rescrito por:

$$
\left[\begin{array}{c}
\Delta V_{1} \\
\Delta V_{2} \\
\vdots \\
\Delta V_{k}
\end{array}\right]=\left[\begin{array}{cccc}
S_{11} & S_{12} & \cdots & S_{1 m} \\
S_{21} & S_{22} & \cdots & S_{2 m} \\
\vdots & \vdots & \ddots & \vdots \\
S_{k 1} & S_{k 2} & \cdots & S_{k m}
\end{array}\right]\left[\begin{array}{c}
\Delta Q_{1} \\
\Delta Q_{2} \\
\vdots \\
\Delta Q_{m}
\end{array}\right]
$$

Sendo $k$ o número de barras de carga e $m$ o número de barras onde serão alocados reativos. 
Devido à alocação de reativos no sistema, uma variação $\Delta V_{i}$ nas tensões $V_{i}$ das barras de carga $i$, fará com que as tensões fiquem dentro de seus limites, ou seja:

$$
V_{i}^{\min } \leq V_{i}+\Delta V_{i} \leq V_{i}^{\max }
$$

Sendo $V_{i}^{\min }$ e $V_{i}^{\max }$ os valores mínimos e máximos desejados nas barras de carga $i$. Desmembrando a equação (4.17) e substituindo em (4.16), tem-se:

$$
\begin{aligned}
& S_{i 1} \Delta Q_{1}+S_{i 2} \Delta Q_{2}+\ldots+S_{i m} \Delta Q_{m} \geq V_{i}^{m i n}-V_{i} \\
& S_{i 1} \Delta Q_{1}+S_{i 2} \Delta Q_{2}+\ldots+S_{i m} \Delta Q_{m} \leq V_{i}^{m a ́ x}-V_{i}
\end{aligned}
$$

A formulação do problema de alocação ótima de reativos utilizando programação linear, como será apresentado neste trabalho, pode ser representada por:

$$
\begin{array}{ll}
\text { Min } & F(Q)=\sum_{j=l}^{m} \Delta Q_{j} \\
\text { s.a. } & {[S][\Delta Q] \geq V_{i}^{\min }-V_{i}} \\
& {[S][\Delta Q] \leq V_{i}^{\max }-V_{i}} \\
& \Delta Q_{j} \geq 0 \\
& j=1, \ldots, m
\end{array}
$$

Sendo:

$F(Q)$ a função objetivo a ser minimizada;

$\Delta Q_{j}$ a variação de potência reativa das barras à alocação de reativos, obtidas a partir do programa de fluxo de potência ótimo; $m$ o número de barras a serem alocados reativos;

$[S]$ a matriz de sensibilidade;

$[\Delta Q]$ o vetor das variáveis independentes. 
A função objetivo do problema de programação linear apresentado pela equação (4.19) representa a variação de potência reativa alocada nas barras de $j$ a $m$. As barras são determinadas a partir dos valores dos multiplicadores de Lagrange $(\lambda)$ associados às restrições de igualdade de potência reativa $(\Delta Q=0)$, e resultantes do processo de otimização realizado a partir do programa de fluxo de carga ótimo, como mencionado anteriormente. A matriz de sensibilidade, para o problema proposto, será calculada a partir da equação (4.9). 


\section{TESTES E RESULTADOS}

\section{1) Introdução}

Mostraremos, a seguir, os resultados obtidos utilizando a nova abordagem proposta para a resolução do problema de planejamento de reativos. Para os sistemas apresentados foram feitos dois testes distintos a fim de certificar a consistência dos resultados apresentados. Primeiramente foi feito um teste utilizando um método clássico, proposto por KISHORE (1970). Em seguida utilizou-se a abordagem proposta.

Os testes foram realizados nos sistemas WARD \& HALE (W\&H), 8, 30 e 118 barras. Os limites máximo e mínimo de tensão utilizados para os sistemas foram 1.1 p.u e 0.95 p.u., respectivamente. Os testes foram realizados em um microcomputador Pentium III - 500MHz, com 128 Mbytes de memória RAM, do Laboratório de Otimização em Sistemas Elétricos de Potência (LOSEP), do Departamento de Engenharia Elétrica da Escola de Engenharia Elétrica de São Carlos (EESC - USP). 


\section{2) Sistema WARD \& HALE}

O sistema WARD \& HALE, utilizado para realização dos testes, é apresentado na Figura1. O sistema apresentado possui as seguintes características:

- 1 barra de referência;

- 1 barra de geração;

- 4 barras de carga;

- 7 linhas de transmissão.

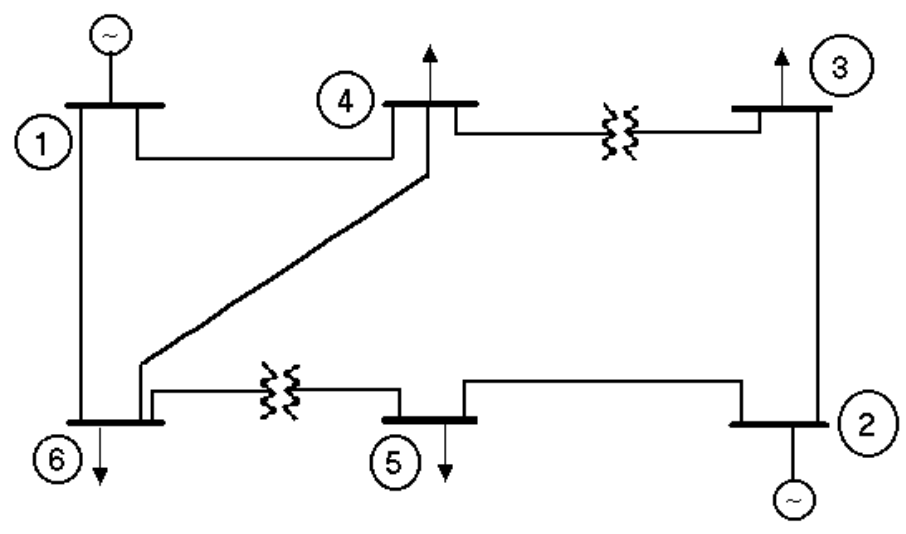

Figura 1- Sistema WARD \& HALE

Os dados iniciais de tensões, gerações e cargas do sistema são apresentados na Tabela 1. 
Tabela 1 - Dados iniciais do sistema WARD \& HALE

\begin{tabular}{|c|c|c|c|c|c|c|c|}
\hline \multirow{2}{*}{ Barra } & \multirow{2}{*}{ Tipo } & \multicolumn{2}{|c|}{ Tensão } & \multicolumn{2}{c|}{ Geração } & \multicolumn{2}{c|}{ Carga } \\
\cline { 3 - 8 } & & $\mathrm{V}$ & $\theta$ & $\mathrm{P}$ & $\mathrm{Q}$ & $\mathrm{P}$ & $\mathrm{Q}$ \\
(p.u.) & $\begin{array}{c}\theta \\
\text { (graus) }\end{array}$ & (MW) & (MVAr) & (MW) & (MVAr) \\
\hline 1 & Referência & 1,05 & 0,0 & - & - & - & - \\
\hline 2 & Geração & 1,10 & - & 50,0 & - & 0,0 & 0,0 \\
\hline 3 & Carga & 1,00 & - & - & - & 55,0 & 13,0 \\
\hline 4 & Carga & 1,00 & - & - & - & 0,0 & 0,0 \\
\hline 5 & Carga & 1,00 & - & - & - & 30,0 & 18,0 \\
\hline 6 & Carga & 1,00 & - & - & - & 50,0 & 5,0 \\
\hline
\end{tabular}

A tabela 2 apresenta o estado final do sistema WARD \& HALE, obtido via fluxo de carga. O programa de fluxo de carga utilizado foi o ANAREDE ${ }^{1}$.

Tabela 2 - Ponto de operação do sistema WARD \& HALE, resultante do fluxo de carga convencional

\begin{tabular}{|c|c|c|c|c|c|c|c|}
\hline \multirow{2}{*}{ Barra } & \multirow{2}{*}{ Tipo } & \multicolumn{2}{|c|}{ Tensão } & \multicolumn{2}{c|}{ Geração } & \multicolumn{2}{c|}{ Carga } \\
\cline { 3 - 8 } & & $\begin{array}{c}\mathrm{V} \\
\text { (p.u.) }\end{array}$ & $\begin{array}{c}\theta \\
\text { (graus) }\end{array}$ & $\begin{array}{c}\mathrm{P} \\
\text { (MW) }\end{array}$ & $\begin{array}{c}\text { Q } \\
\text { (MVAr) }\end{array}$ & $\begin{array}{c}\text { P } \\
\text { (MW) }\end{array}$ & $\begin{array}{c}\text { Q } \\
\text { (MVAr) }\end{array}$ \\
\hline 1 & Referência & 1,0500 & 0,0000 & 96,6123 & 38,1103 & - & - \\
\hline 2 & Geração & 1,1000 & $-6,1424$ & 50,0000 & 34,8010 & 0,0 & 0,0 \\
\hline 3 & Carga & 0,8552 & $-13,8286$ & - & - & 55,0 & 13,0 \\
\hline 4 & Carga & 0,9526 & $-9,9223$ & - & - & 0,0 & 0,0 \\
\hline 5 & Carga & 0,9009 & $-13,4223$ & - & - & 30,0 & 18,0 \\
\hline 6 & Carga & 0,9332 & 0,0000 & - & - & 50,0 & 5,0 \\
\hline
\end{tabular}

Pode-se observar que as tensões nas barras 3, 5 e 6 estão fora de seus limites especificados, fazendo-se necessário alocar reativos para que as tensões fiquem

\footnotetext{
${ }^{1}$ O programa de fluxo de carga ANAREDE foi desenvolvido pelo CEPEL - Centro de Pesquisas de Energia Elétrica.
} 
dentro de seus limites. Determinada a necessidade de se alocarem reativos, foram feitos os dois testes com o intuito de certificar a validade do resultado apresentado como solução do problema.

O primeiro teste foi realizado a partir do método apresentado por KISHORE (1970), em que todas as barras de carga são candidatas à alocação de reativos. Para o ponto de operação deste sistema, uma matriz sensibilidade foi determinada a partir da matriz Jacobiana representada pela Equação 4.9. Os coeficientes desta matriz são usados para construir o problema de programação linear segundo a Equação 4.19, resultando em:

$$
\begin{aligned}
& \begin{array}{l}
\text { Minimizar } \Delta Q_{3}+\Delta Q_{4}+\Delta Q_{5}+\Delta Q_{6} \\
\text { sujeito à }
\end{array} \\
& 0,3477 \Delta Q_{3}+0,2127 \Delta Q_{4}+0,0789 \Delta Q_{5}+0,1046 \Delta Q_{6} \geq 0,092 \\
& 0,2368 \Delta Q_{3}+0,2493 \Delta Q_{4}+0,0935 \Delta Q_{5}+0,1226 \Delta Q_{6} \geq-0,005 \\
& 0,0820 \Delta Q_{3}+0,0864 \Delta Q_{4}+0,4062 \Delta Q_{5}+0,2038 \Delta Q_{6} \geq 0,048 \\
& 0,1115 \Delta Q_{3}+0,1174 \Delta Q_{4}+0,2112 \Delta Q_{5}+0,2770 \Delta Q_{6} \geq 0,015 \\
& 0,3477 \Delta Q_{3}+0,2127 \Delta Q_{4}+0,0789 \Delta Q_{5}+0,1046 \Delta Q_{6} \leq 0,242 \\
& 0,2368 \Delta Q_{3}+0,2493 \Delta Q_{4}+0,0935 \Delta Q_{5}+0,1226 \Delta Q_{6} \leq 0,145 \\
& 0,0820 \Delta Q_{3}+0,0864 \Delta Q_{4}+0,4062 \Delta Q_{5}+0,2038 \Delta Q_{6} \leq 0,198 \\
& 0,1115 \Delta Q_{3}+0,1174 \Delta Q_{4}+0,2112 \Delta Q_{5}+0,2770 \Delta Q_{6} \leq 0,165 \\
& \Delta Q_{3}, \Delta Q_{4}, \Delta Q_{5}, \Delta Q_{6} \geq 0
\end{aligned}
$$

Resolvendo o problema de programação linear com a utilização do software LINDO API, que utiliza como interface o software MATLAB, foram obtidos os resultados mostrados na Tabela 3. A tabela apresenta os novos reativos e as novas tensões obtidas com o fluxo de carga convencional, após a instalação das novas fontes de potência reativa. Observa-se que as tensões atingiram níveis mais adequados para operação do sistema. 
Tabela 3 - Resultado do sistema WARD \& HALE para a alocação reativa nas barras de carga do sistema

\begin{tabular}{|c|c|c|c|c|c|c|c|c|}
\hline Barra & \multirow{2}{*}{ Tipo } & \multicolumn{2}{|c|}{ Tensão } & \multicolumn{2}{c|}{ Geração } & \multicolumn{2}{c|}{ Carga } & Qsh \\
\cline { 3 - 8 } & & $\begin{array}{c}\mathrm{V} \\
\text { (p.u.) }\end{array}$ & $\begin{array}{c}\theta \\
\text { (graus) }\end{array}$ & $\begin{array}{c}\mathrm{P} \\
(\mathrm{MW})\end{array}$ & $\begin{array}{c}\mathrm{Q} \\
\text { (MVAr) }\end{array}$ & $\begin{array}{c}\mathrm{P} \\
(\mathrm{MW})\end{array}$ & $\begin{array}{c}\mathrm{Q} \\
\text { (MVAr) }\end{array}$ & $\begin{array}{c}\text { (MVAr) } \\
\text { (alocado) }\end{array}$ \\
\hline 1 & Referência & 1,0500 & 0,000 & 94,4023 & 14,1957 & - & - & - \\
\hline 2 & Geração & 1,1000 & $-4,038$ & 50,0000 & 20,3751 & 0,0 & 0,0 & - \\
\hline 3 & Carga & 0,9376 & $-13,348$ & - & 25,5300 & 55,0 & 13,0 & 25,5300 \\
\hline 4 & Carga & 1,0112 & $-9,982$ & - & - & 0,0 & 0,0 & - \\
\hline 5 & Carga & 0,9422 & $-12,522$ & - & 6,8900 & 30,0 & 18,0 & 6,8900 \\
\hline 6 & Carga & 0,9722 & $-12,123$ & - & - & 50,0 & 5,0 & - \\
\hline \multicolumn{7}{|l|}{ PERDAS = 9,40MW }
\end{tabular}

Realizado o primeiro teste, foram feitos novos testes seguindo a abordagem proposta. A abordagem proposta utiliza os multiplicadores de Lagrange, resultantes da solução do fluxo de potência ótimo, para determinar as barras onde serão alocados reativos. A partir de uma análise aos multiplicadores de Lagrange, concluiu-se que a barra que possui uma maior sensibilidade à variação de potência reativa do sistema é a barra 5, como apresentado na Tabela 4 .

Tabela 4 - Solução do sistema WARD \& HALE resultante do Fluxo de Potência Ótimo

\begin{tabular}{|c|c|c|c|c|c|c|}
\hline \multirow{2}{*}{ Barra } & \multirow{2}{*}{ Tipo } & \multicolumn{2}{|c|}{ Tensão } & \multicolumn{2}{|c|}{ Potência } & \multirow{2}{*}{$\lambda_{\mathrm{Q}}$} \\
\cline { 3 - 6 } & $\mathrm{V}$ & $\begin{array}{c}\theta \\
\text { (p.u.) }\end{array}$ & $\begin{array}{c}\mathrm{P} \\
\text { (graus) }\end{array}$ & $\begin{array}{c}\mathrm{Q} \\
\text { (MW) }\end{array}$ & - \\
\hline 1 & Referência & 1,093 & 0.0 & 95,0 & 53,2 & - \\
\hline 2 & Geração & 1,086 & $-2,3$ & 50,0 & 17,3 & 0,09769 \\
\hline 3 & Carga & 0,955 & $-12,1$ & $-55,0$ & $-13,0$ & 0,09157 \\
\hline 4 & Carga & 0,968 & $-8,9$ & 0,0 & 0,0 & 0,14396 \\
\hline 5 & Carga & 0,928 & $-11,4$ & $-30,0$ & $-18,0$ & 0,11837 \\
\hline 6 & Carga & 0,948 & $-11,2$ & $-50,0$ & $-5,0$ & \\
\hline \multicolumn{7}{|l|}{ PERDAS = 10,043W }
\end{tabular}


Formulando o problema de programação linear, como em KISHORE (1970), utilizando os dados referentes à barra 5, provenientes do fluxo de carga convencional, tem-se:

$$
\begin{aligned}
& \begin{array}{l}
\text { Minimizar } \Delta Q_{5} \\
\text { sujeito à }
\end{array} \\
& 0,4062 \Delta Q_{5} \geq 0,048 \\
& 0,4062 \Delta Q_{5} \leq 0,198 \\
& \Delta Q_{5} \geq 0
\end{aligned}
$$

\begin{tabular}{|c|c|c|c|c|c|c|c|c|}
\hline \multirow[t]{2}{*}{ Barra } & \multirow[t]{2}{*}{ Tipo } & \multicolumn{2}{|c|}{ Tensão } & \multicolumn{2}{|c|}{ Geração } & \multicolumn{2}{|c|}{ Carga } & \multirow{2}{*}{$\begin{array}{c}\mathrm{Q}_{\mathrm{sh}} \\
\text { (MVAr) } \\
\text { (alocado) }\end{array}$} \\
\hline & & $\begin{array}{c}\mathrm{V} \\
\text { (p.u.) }\end{array}$ & $\begin{array}{c}\theta \\
\text { (graus) }\end{array}$ & $\begin{array}{c}\mathrm{P} \\
(\mathrm{MW})\end{array}$ & $\begin{array}{c}\mathrm{Q} \\
\text { (MVAr) }\end{array}$ & $\begin{array}{c}\mathrm{P} \\
(\mathrm{MW})\end{array}$ & $\begin{array}{c}\mathrm{Q} \\
\text { (MVAr) }\end{array}$ & \\
\hline 1 & Referência & 1,0500 & 0,000 & 95,5758 & 30,9166 & - & - & - \\
\hline 2 & Geração & 1,1000 & $-5,316$ & 50,0000 & 26,8454 & 0,0 & 0,0 & - \\
\hline 3 & Carga & 0,8630 & $-13,584$ & - & - & 55,0 & 13,0 & - \\
\hline 4 & Carga & 0,9625 & $-9,7946$ & - & - & 0,0 & 0,0 & - \\
\hline 5 & Carga & 0,9444 & $-13,227$ & - & 12,0700 & 30,0 & 18,0 & 12,07 \\
\hline 6 & Carga & 0,9559 & $-12,565$ & - & - & 50,0 & 5,0 & - \\
\hline
\end{tabular}

O problema de programação linear representado pela Equação 5.2 tem como solução:

Tabela 5 - Resultado do sistema WARD \& HALE para a alocação reativa na barra 5, resultante do problema de programação linear

A partir da quantidade de reativo alocado, apresentado pela Tabela 5, realizase um novo teste, através do fluxo de carga, acrescentando aos dados iniciais essa potência reativa alocada e novamente se faz um estudo de análise aos multiplicadores de Lagrange. Neste novo estudo, a solução do fluxo de potência ótimo apresentou 
uma maior sensibilidade à variação de potência reativa do sistema, para o multiplicador de Lagrange que representa a barra 3, como mostrado na Tabela 6 .

Tabela 6 - Solução do sistema WARD \& HALE resultante do Fluxo de Potência Ótimo após a injeção de reativos na barra 5

\begin{tabular}{|c|c|c|c|c|c|c|}
\hline \multirow{2}{*}{ Barra } & \multirow{2}{*}{ Tipo } & \multicolumn{2}{|c|}{ Tensão } & \multicolumn{2}{c|}{ Potência } & \multirow{2}{*}{$\lambda_{\mathrm{Q}}$} \\
\cline { 3 - 7 } & & $\mathrm{V}$ & $\theta$ & $\mathrm{P}$ & $\mathrm{Q}$ \\
(p.u.) & (graus) & (MW) & (MVAr) & \\
\hline 1 & Referência & 1,083 & 0,0 & 94,5 & 45,8 & - \\
\hline 2 & Geração & 1,080 & $-1,9$ & 50,0 & 10,3 & - \\
\hline 3 & Carga & 0,953 & $-12,2$ & $-55,0$ & $-13,0$ & 0,08154 \\
\hline 4 & Carga & 0,967 & $-9,0$ & 0,0 & 0,0 & 0,07461 \\
\hline 5 & Carga & 0,962 & $-11,6$ & $-30,0$ & $-5,9$ & 0,07415 \\
\hline 6 & Carga & 0,960 & $-11,4$ & $-50,0$ & $-5,0$ & 0,07362 \\
\hline \multicolumn{7}{|l|}{ PERDAS =9,467MW }
\end{tabular}

Formulando um novo problema de programação linear com dados provenientes da barra 3, resultante do fluxo de carga convencional, obteve-se:

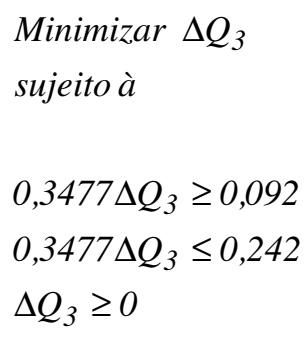

A solução do problema de programação linear representada pela Equação 5.3, acrescida da solução apresentada pela equação 5.2, pode ser analisado na Tabela 7. 
Tabela 7 - Resultado do sistema WARD \& HALE para a alocação reativa nas barras 3 e 5

\begin{tabular}{|c|c|c|c|c|c|c|c|c|}
\hline \multirow{2}{*}{ Barra } & Tipo & \multicolumn{2}{|c|}{ Tensão } & \multicolumn{2}{c|}{ Geração } & \multicolumn{2}{c|}{ Carga } & Q \\
\cline { 3 - 8 } & & $\begin{array}{c}\mathrm{V} \\
\text { (p.u.) }\end{array}$ & $\begin{array}{c}\theta \\
\text { (graus) }\end{array}$ & $\begin{array}{c}\mathrm{P} \\
(\mathrm{MW})\end{array}$ & $\begin{array}{c}\mathrm{Q} \\
(\mathrm{MVAr})\end{array}$ & $\begin{array}{c}\mathrm{P} \\
(\mathrm{MW})\end{array}$ & $\begin{array}{c}\mathrm{Q} \\
(\mathrm{MVAr})\end{array}$ & $\begin{array}{c}\text { (MVAr) } \\
\text { (alocado) }\end{array}$ \\
\hline 1 & Referência & 1,0500 & 0,000 & 94,0824 & 10,2575 & - & - & - \\
\hline 2 & Geração & 1,1000 & $-3,697$ & 50,0000 & 16,6364 & 0,0 & 0,0 & - \\
\hline 3 & Carga & 0,9449 & $-13,269$ & - & 27,1100 & 55,0 & 13,0 & 27,11 \\
\hline 4 & Carga & 1,0179 & $-9,959$ & - & - & 0,0 & 0,0 & - \\
\hline 5 & Carga & 0,9608 & $-12,458$ & - & 12,0700 & 30,0 & 18,0 & 12,07 \\
\hline 6 & Carga & 0,9827 & $-12,096$ & - & - & 50,0 & 5,0 & - \\
\hline \multicolumn{7}{|l|}{ PERDAS =9,08MW }
\end{tabular}

Para uma melhor visão da evolução dos valores de tensão para os testes realizados, segundo abordagem proposta, para o sistema WARD \& HALE, foi traçado o gráfico representado pela Figura 2. Os valores de potência reativa alocada apresentado na Figura 2 são valores acumulativos, provenientes da solução do problema de programação linear.

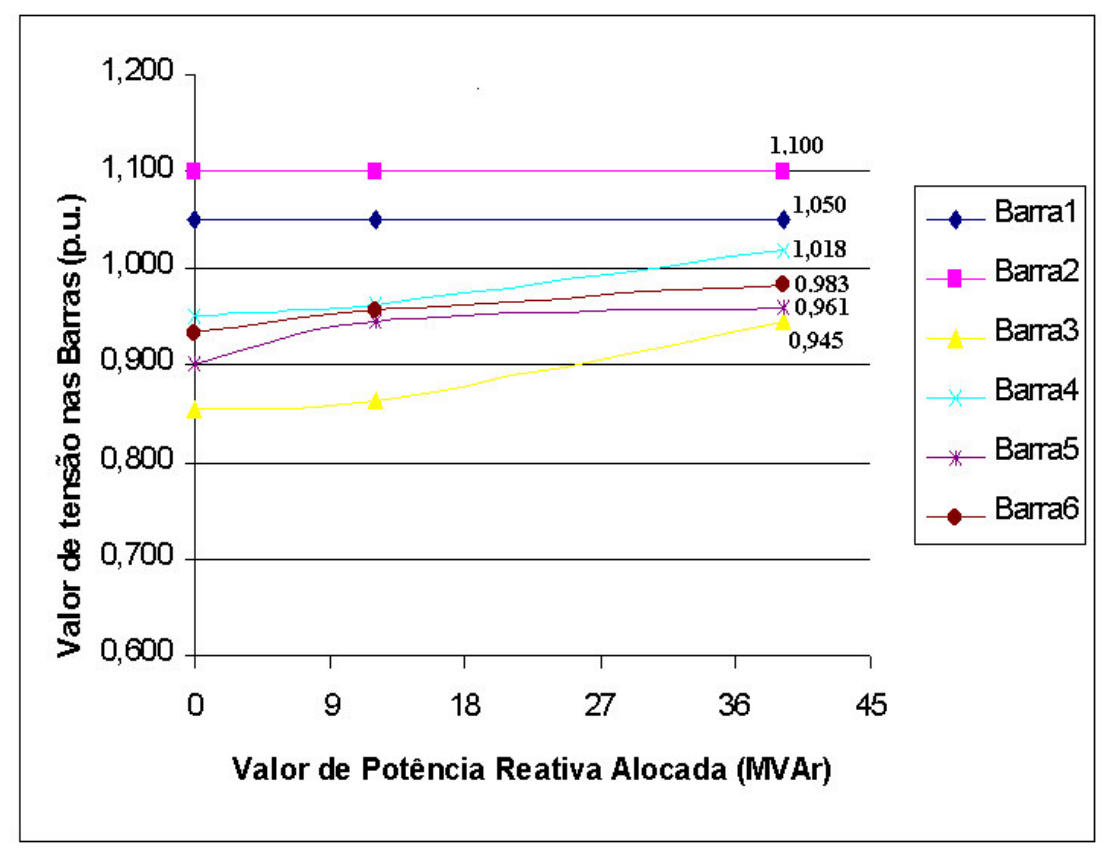

Figura 2 - Gráfico representando os testes realizados segundo abordagem proposta 
Um novo estudo de análise aos multiplicadores de Lagrange e uma nova formulação do problema de programação linear se repetem até que a solução apresentada não modifique o ponto de operação do sistema.

Este exemplo mostra que o conhecimento prévio das barras determinadas para a alocação de reativos traz uma grande economia na formulação do problema de programação linear, sem que se perca na qualidade dos resultados. Outro fato que deve ser considerado é que caso haja o conhecimento prévio das barras, a formulação do problema de programação linear se torna ainda mais simples trazendo assim respostas que melhorem ainda mais o ponto de operação do sistema. 


\section{2) Sistema de 8 Barras}

O sistema de 8 barras (Figura 3), utilizado para realização dos testes, possui as seguintes características:

- 1 barra de referência;

- 1 barra de geração;

- 6 barras de carga;

- 9 linhas de transmissão.

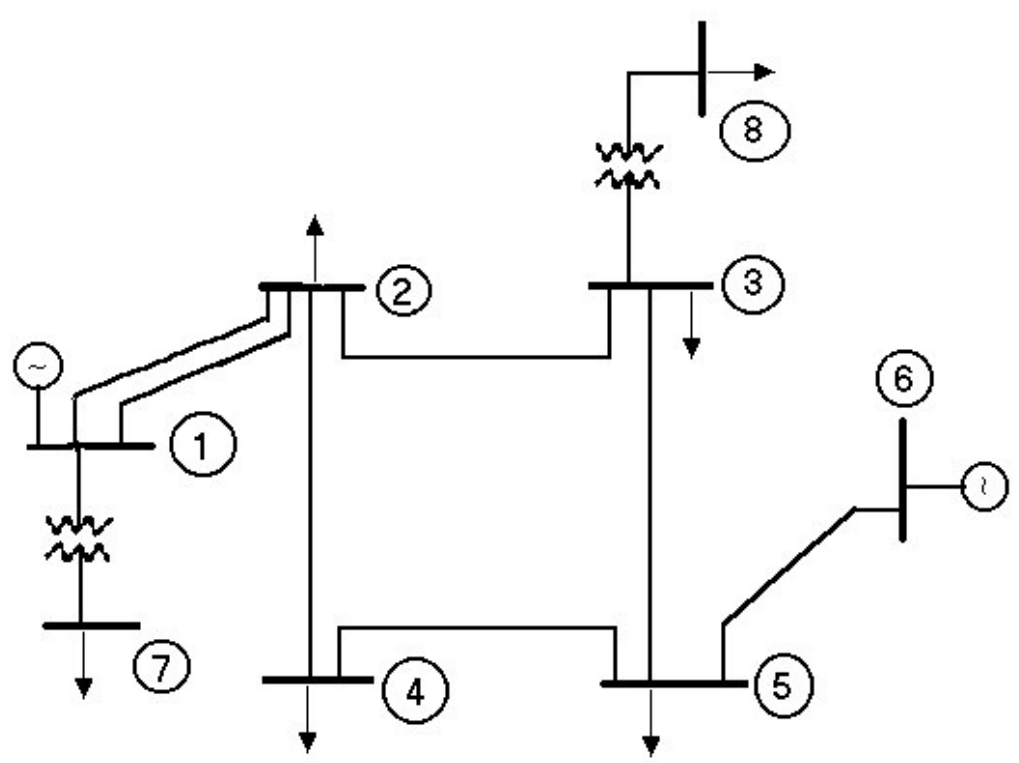

Figura 3- Sistema de 8 barras

Os dados iniciais de tensões, gerações e cargas para sistema de 8 barras são apresentados na Tabela 8 . 
Tabela 8 - Dados iniciais do sistema de 8 barras

\begin{tabular}{|c|c|c|c|c|c|c|c|c|}
\hline \multirow{2}{*}{ Barra } & Tipo & \multicolumn{2}{|c|}{ Tensão } & \multicolumn{2}{c|}{ Geração } & \multicolumn{3}{c|}{ Carga } \\
\cline { 3 - 9 } & & V (p.u.) & $\begin{array}{c}\theta \\
\text { (graus) }\end{array}$ & P (MW) & $\begin{array}{c}\text { Q } \\
\text { (MVAr) }\end{array}$ & P (MW) & $\begin{array}{c}\text { Q } \\
\text { (MVAr) }\end{array}$ & $\begin{array}{c}\text { Q }_{\text {sh }} \\
\text { (MVAr) }\end{array}$ \\
\hline 1 & Referência & 1,05 & 0,0 & - & - & 0,0 & 0,0 & - \\
\hline 2 & Carga & 1,00 & - & - & - & 0,0 & 0,0 & $-300,0$ \\
\hline 3 & Carga & 1,00 & - & - & - & 0,0 & 0,0 & $-75,0$ \\
\hline 4 & Carga & 1,00 & - & - & - & 134,0 & $-32,0$ & $-140,0$ \\
\hline 5 & Carga & 1,00 & - & - & - & 1300,0 & 50,0 & $-140,0$ \\
\hline 6 & Geração & 1,00 & - & 240,0 & - & 0,0 & 0,0 & - \\
\hline 7 & Carga & 1,00 & - & - & - & 180,0 & 86,0 & - \\
\hline 8 & Carga & 1,00 & - & - & - & 290,0 & 48,0 & - \\
\hline
\end{tabular}

A Tabela 9 apresenta o estado final do sistema de 8 barras. Para o sistema representado pela Figura 3, têm-se os seguintes valores resultantes do fluxo de carga convencional.

Tabela 9 - Ponto de operação do sistema de 8 barras, resultante do fluxo de carga convencional

\begin{tabular}{|c|c|c|c|c|c|c|c|}
\hline \multirow{2}{*}{ Barra } & Tipo & \multicolumn{2}{|c|}{ Tensão } & \multicolumn{2}{c|}{ Geração } & \multicolumn{2}{c|}{ Carga } \\
\cline { 3 - 8 } & & $\begin{array}{c}\mathrm{V} \\
\text { (p.u.) }\end{array}$ & $\begin{array}{c}\theta \\
\text { (graus) }\end{array}$ & $\begin{array}{c}\mathrm{P} \\
(\mathrm{MW})\end{array}$ & $\begin{array}{c}\mathrm{Q} \\
(\mathrm{MVAr})\end{array}$ & $\begin{array}{c}\mathrm{P} \\
(\mathrm{MW})\end{array}$ & $\begin{array}{c}\text { Q } \\
\text { (MVAr) }\end{array}$ \\
\hline 1 & Referência & 1,0500 & 0,0000 & 1711,9291 & 236,3154 & - & - \\
\hline 2 & Carga & 1,0068 & $-11,9714$ & - & - & - & - \\
\hline 3 & Carga & 0,9903 & $-23,5582$ & - & - & - & - \\
\hline 4 & Carga & 0,9953 & $-23,0094$ & - & - & 134,0 & $-32,0$ \\
\hline 5 & Carga & 0,9917 & $-32,7981$ & - & - & 1300,0 & 50,0 \\
\hline 6 & Geração & 1,0000 & $-31,4360$ & 240,00 & 28,6728 & - & - \\
\hline 7 & Carga & 1,0291 & $-2,2913$ & - & - & 180,00 & 86,00 \\
\hline 8 & Carga & 0,8837 & $-28,5983$ & - & - & 290,00 & 48,00 \\
\hline \multicolumn{7}{|l|}{ PERDAS =47,93MW } \\
\hline
\end{tabular}


No primeiro teste realizado para o sistema de oito barras, a função objetivo do problema de programação linear foi minimizar a injeção de potência reativa de todas as barras de carga. A matriz sensibilidade foi calculada a partir da matriz Jacobiana, e o problema de programação linear foi formulado a partir dos dados obtidos na Tabela 9, sendo representado por:

$$
\begin{aligned}
& \begin{array}{l}
\text { Minimizar } \Delta Q_{2}+\Delta Q_{3}+\Delta Q_{4}+\Delta Q_{5}+\Delta Q_{7}+\Delta Q_{8} \\
\text { sujeito à }
\end{array} \\
& 0,0266 \Delta Q_{2}+0,0171 \Delta Q_{3}+0,0160 \Delta Q_{4}+0,0064 \Delta Q_{5}+0,0 \Delta Q_{7}+0,0176 \Delta Q_{8} \geq-0,0568 \\
& 0,0164 \Delta Q_{2}+0,0259 \Delta Q_{3}+0,0112 \Delta Q_{4}+0,0070 \Delta Q_{5}+0,0 \Delta Q_{7}+0,0266 \Delta Q_{8} \geq-0,0403 \\
& 0,0155 \Delta Q_{2}+0,0153 \Delta Q_{3}+0,0239 \Delta Q_{4}+0,0070 \Delta Q_{5}+0,0 \Delta Q_{7}+0,0116 \Delta Q_{8} \geq-0,0453 \\
& 0,0060 \Delta Q_{2}+0,0069 \Delta Q_{3}+0,0068 \Delta Q_{4}+0,0086 \Delta Q_{5}+0,0 \Delta Q_{7}+0,0071 \Delta Q_{8} \geq-0,0417 \\
& 0.0 \Delta Q_{2}+\quad 0,0 \Delta Q_{3}+\quad 0,0 \Delta Q_{4}+0,0 \Delta Q_{5}+0,0238 \Delta Q_{7}+0,0 \Delta Q_{8} \geq-0,0791 \\
& 0,0151 \Delta Q_{2}+0,0238 \Delta Q_{3}+0,0103 \Delta Q_{4}+0,0064 \Delta Q_{5}+0,0 \Delta Q_{7}+0,0522 \Delta Q_{8} \geq 0,0663 \\
& 0,0266 \Delta Q_{2}+0,0171 \Delta Q_{3}+0,0160 \Delta Q_{4}+0,0064 \Delta Q_{5}+0,0 \Delta Q_{7}+0,0176 \Delta Q_{8} \leq 0,0932 \\
& 0,0164 \Delta Q_{2}+0,0259 \Delta Q_{3}+0,0112 \Delta Q_{4}+0,0070 \Delta Q_{5}+0,0 \Delta Q_{7}+0,0266 \Delta Q_{8} \leq 0,1097 \\
& 0,0155 \Delta Q_{2}+0,0153 \Delta Q_{3}+0,0239 \Delta Q_{4}+0,0070 \Delta Q_{5}+0,0 \Delta Q_{7}+0,0116 \Delta Q_{8} \leq 0,1047 \\
& 0,0060 \Delta Q_{2}+0,0069 \Delta Q_{3}+0,0068 \Delta Q_{4}+0,0086 \Delta Q_{5}+0,0 \Delta Q_{7}+0,0071 \Delta Q_{8} \leq 0,1083 \\
& 0,0 \Delta Q_{2}+\quad 0,0 \Delta Q_{3}+\quad 0,0 \Delta Q_{4}+\quad 0,0 \Delta Q_{5}+0,0238 \Delta Q_{7}+0,0 \Delta Q_{8} \leq 0,0709 \\
& 0,0151 \Delta Q_{2}+0,0238 \Delta Q_{3}+0,0103 \Delta Q_{4}+0,0064 \Delta Q_{5}+0,0 \Delta Q_{7}+0,0522 \Delta Q_{8} \leq 0,2163 \\
& \Delta Q_{2}, \Delta Q_{3}, \Delta Q_{4}, \Delta Q_{5}, \Delta Q_{7}, \Delta Q_{8} \geq 0
\end{aligned}
$$

Resolvendo o problema de programação linear, foram obtidos os resultados mostrados na Tabela 10. Pode ser observado que, devido à alocação de reativos na barra 8 , as tensões atingiram níveis mais adequados. 
Tabela 10 - Resultado do sistema de 8 barras para a alocação reativa nas barras de carga do sistema

\begin{tabular}{|c|c|c|c|c|c|c|c|c|}
\hline \multirow[t]{2}{*}{ Barra } & \multirow[t]{2}{*}{ Tipo } & \multicolumn{2}{|c|}{ Tensão } & \multicolumn{2}{|c|}{ Geração } & \multicolumn{2}{|c|}{ Carga } & \multirow{2}{*}{$\begin{array}{c}\mathrm{Q}_{\mathrm{sh}} \\
\text { (MVAr) } \\
\text { (alocado) }\end{array}$} \\
\hline & & $\begin{array}{c}\mathrm{V} \\
\text { (p.u.) }\end{array}$ & $\begin{array}{c}\theta \\
\text { (graus) }\end{array}$ & $\begin{array}{c}\mathrm{P} \\
(\mathrm{MW})\end{array}$ & $\begin{array}{c}\mathrm{Q} \\
\text { (MVAr) }\end{array}$ & $\begin{array}{c}\mathrm{P} \\
(\mathrm{MW})\end{array}$ & $\begin{array}{c}\mathrm{Q} \\
\text { (MVAr) }\end{array}$ & \\
\hline 1 & Referência & 1,0500 & 0,0000 & 1710,56 & 151,534 & - & - & - \\
\hline 2 & Carga & 1,0183 & $-11,8618$ & - & - & - & - & - \\
\hline 3 & Carga & 1,0206 & $-23,1164$ & - & - & - & - & - \\
\hline 4 & Carga & 1,0062 & $-22,5531$ & - & - & 134,0 & $-32,0$ & - \\
\hline 5 & Carga & 1,0000 & $-32,0426$ & - & - & 1300,0 & 50,0 & - \\
\hline 6 & Geração & 1,0000 & $-30,6634$ & 240,0 & $-53,931$ & - & - & - \\
\hline 7 & Carga & 1,0291 & $-2,2913$ & - & - & 180,0 & 86,0 & - \\
\hline 8 & Carga & 0,9450 & $-27,6885$ & - & 127,10 & 290,0 & 48,0 & 127,10 \\
\hline וDי & 16571 & & & & & & & \\
\hline
\end{tabular}

Um novo teste foi realizado aplicando-se a abordagem proposta. A partir dos resultados obtidos na Tabela 11 foi feito um estudo dos multiplicadores de Lagrange. Este estudo determinou a barra 8 como sendo a de maior sensibilidade à variação de potência reativa.

Tabela 11 - Solução do sistema de 8 barras, resultante do Fluxo de Potência Ótimo

\begin{tabular}{|c|c|c|c|c|c|c|}
\hline \multirow[t]{2}{*}{ Barra } & \multirow[t]{2}{*}{ Tipo } & \multicolumn{2}{|c|}{ Tensão } & \multicolumn{2}{|c|}{ Potência } & \multirow[b]{2}{*}{$\lambda_{\mathrm{Q}}$} \\
\hline & & $\begin{array}{c}\mathrm{V} \\
\text { (p.u.) }\end{array}$ & $\begin{array}{c}\theta \\
\text { (graus) }\end{array}$ & $\begin{array}{c}\mathrm{P} \\
(\mathrm{MW})\end{array}$ & $\begin{array}{c}\mathrm{Q} \\
\text { (MVAr) }\end{array}$ & \\
\hline 1 & Referência & 1,068 & 0,0 & 1708,4 & 127,5 & - \\
\hline 2 & Carga & 1,038 & $-11,4$ & $-0,1$ & $-0,2$ & 0,01232 \\
\hline 3 & Carga & 1,031 & $-22,2$ & 0,0 & $-0,1$ & 0,02171 \\
\hline 4 & Carga & 1,034 & $-21,7$ & $-134,0$ & 31,9 & 0,01310 \\
\hline 5 & Carga & 1,035 & $-30,7$ & $-1299,7$ & $-49,8$ & 0,01181 \\
\hline 6 & Geração & 1,043 & $-29,5$ & 239,9 & 27,3 & - \\
\hline 7 & Carga & 1,023 & $-2,3$ & $-180,0$ & $-86,0$ & 0,00071 \\
\hline 8 & Carga & 0,997 & $-26,2$ & $-290,0$ & $-47,9$ & 0,03424 \\
\hline
\end{tabular}


Formulando o problema de programação linear com dados provenientes da barra 8, obteve-se:

$$
\begin{aligned}
& \begin{array}{l}
\text { Minimizar } \Delta Q_{8} \\
\text { sujeito à }
\end{array} \\
& 0,0522 \Delta Q_{8} \geq 0,0663 \\
& 0,0522 \Delta Q_{8} \leq 0,2163 \\
& \Delta Q_{8} \geq 0
\end{aligned}
$$

\begin{tabular}{|c|c|c|c|c|c|c|c|c|}
\hline \multirow[t]{2}{*}{ Barra } & \multirow[t]{2}{*}{ Tipo } & \multicolumn{2}{|c|}{ Tensão } & \multicolumn{2}{|c|}{ Geração } & \multicolumn{2}{|c|}{ Carga } & \multirow{2}{*}{$\begin{array}{c}\mathrm{Q}_{\mathrm{sh}} \\
\text { (MVAr) } \\
\text { (alocado) }\end{array}$} \\
\hline & & $\begin{array}{c}\mathrm{V} \\
\text { (p.u.) }\end{array}$ & $\begin{array}{c}\theta \\
\text { (graus) }\end{array}$ & $\begin{array}{c}\mathrm{P} \\
(\mathrm{MW})\end{array}$ & $\begin{array}{c}\mathrm{Q} \\
\text { (MVAr) }\end{array}$ & $\begin{array}{c}\mathrm{P} \\
(\mathrm{MW})\end{array}$ & $\begin{array}{c}\mathrm{Q} \\
\text { (MVAr) }\end{array}$ & \\
\hline 1 & Referência & 1,0500 & 0,000 & 1710,565 & 151,534 & - & - & - \\
\hline 2 & Carga & 1,0183 & $-11,862$ & - & - & - & - & - \\
\hline 3 & Carga & 1,0206 & $-23,116$ & - & - & - & - & - \\
\hline 4 & Carga & 1,0062 & $-22,553$ & - & - & 134,0 & $-32,0$ & - \\
\hline 5 & Carga & 1,0000 & $-32,043$ & - & - & 1300,0 & 50,0 & - \\
\hline 6 & Geração & 1,0000 & $-30,663$ & 240,0 & $-53,931$ & - & - & $\begin{array}{ll}- & -1 \\
\end{array}$ \\
\hline 7 & Carga & 1,0291 & $-2,291$ & - & - & 180,0 & 86,0 & - \\
\hline 8 & Carga & 0,9450 & $-27,688$ & - & 127,10 & 290,0 & 48,0 & 127,10 \\
\hline
\end{tabular}

Determinada a quantidade de reativos a serem instalados, o estado do sistema, via fluxo de carga convencional, é apresentado na Tabela 12.

Tabela 12 - Resultado do sistema de 8 barras para a alocação reativa na barra 8 , segundo método proposto

Os resultados dos testes realizados para o sistema de oito barras apresentaram os mesmos valores, como é possível de se observar nas Tabela 10 e Tabela 12. A 
vantagem em se utilizar o método proposto é o ganho na dimensão do problema de programação linear, como foi mostrado nos testes realizados, e o ganho computacional, visto que a formulação do problema de programação linear se torna mais simples. 


\section{3) Sistema AEP - 30 barras}

O sistema AEP-30 barras, utilizado para realização dos testes, é apresentado na Figura 4. O sistema apresentado possui as seguintes características:

- 1 barra de referência;

- 5 barras de controle de reativos;

- 24 barras de carga;

- 37 linhas de transmissão;

- 4 transformadores com tap variável;

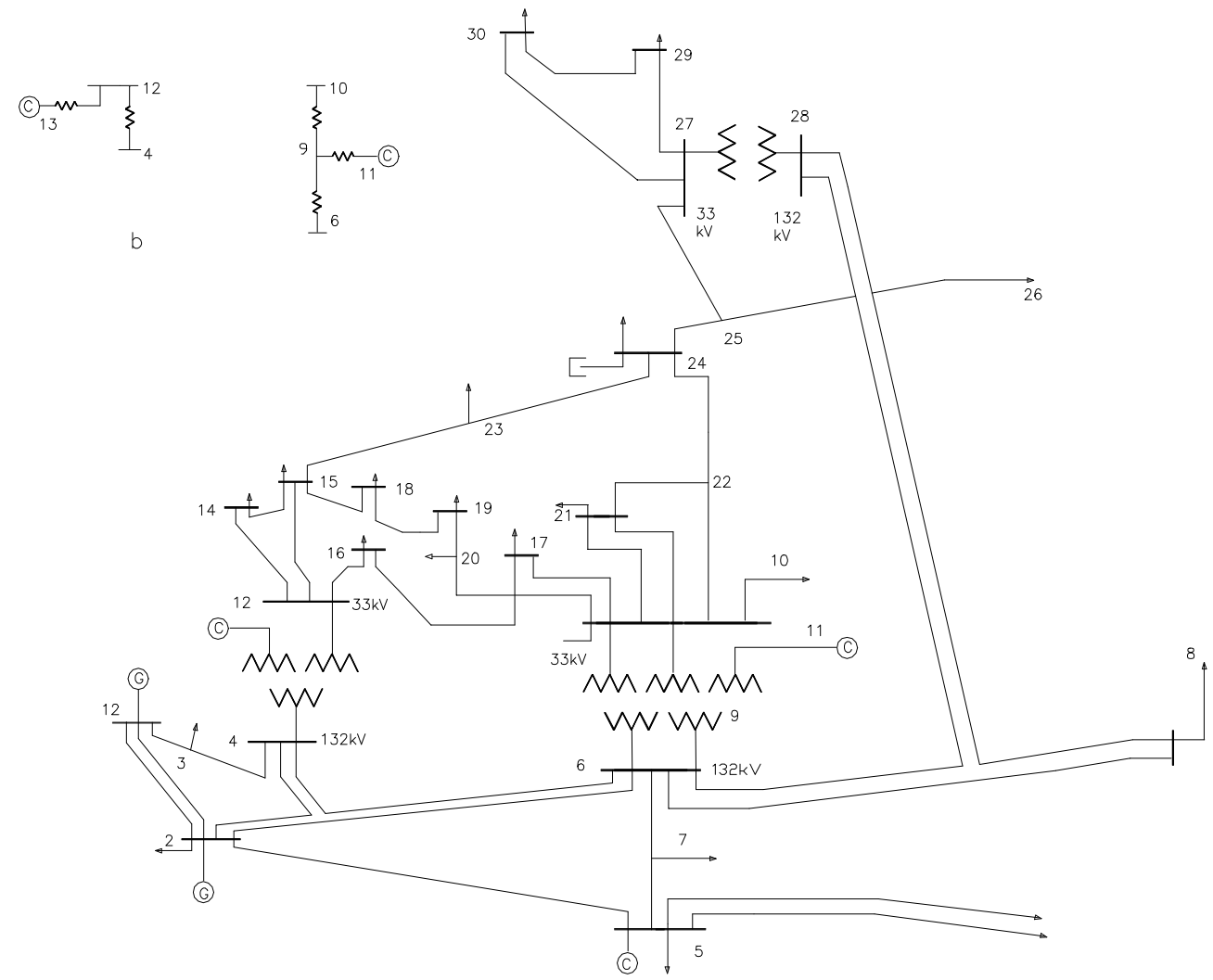

a

Figura 4- Sistema AEP-30 barras 
A Tabela 13 apresenta o estado final do sistema AEP - 30 barras, representado pela Figura 4.

Tabela 13 - Ponto de operação do sistema AEP - 30 barras, resultante do fluxo de carga convencional

\begin{tabular}{|c|c|c|c|c|c|}
\hline \multirow[t]{2}{*}{ Barra } & \multirow[t]{2}{*}{ Tipo } & \multicolumn{2}{|c|}{ Tensão } & \multicolumn{2}{|c|}{ Potência } \\
\hline & & $\begin{array}{c}\mathrm{V} \\
\text { (p.u.) }\end{array}$ & $\begin{array}{c}\theta \\
\text { (graus) }\end{array}$ & $\begin{array}{c}\mathrm{P} \\
(\mathrm{MW})\end{array}$ & $\begin{array}{c}\mathrm{Q} \\
\text { (MVAr) }\end{array}$ \\
\hline 1 & Slack & 1,053 & 0,0 & 290,4 & 17,4 \\
\hline 2 & Gera. & 1,021 & $-6,1$ & 20,1 & 36,0 \\
\hline 3 & Carga & 0,996 & $-9,0$ & $-2,6$ & $-1,3$ \\
\hline 4 & Carga & 0,984 & $-10,8$ & $-8,4$ & $-1,8$ \\
\hline 5 & C. Reat. & 0,975 & $-16,4$ & $-103,6$ & 19,0 \\
\hline 6 & Carga & 0,977 & $-12,8$ & 0,0 & 0,0 \\
\hline 7 & Carga & 0,967 & $-14,9$ & $-25,1$ & $-12,0$ \\
\hline 8 & C. Reat. & 0,975 & $-13,7$ & $-33,0$ & 7,0 \\
\hline 9 & Carga & 0,943 & $-17,2$ & 0,0 & 0,0 \\
\hline 10 & Carga & 0,925 & $-19,5$ & $-6,4$ & $-2,2$ \\
\hline 11 & C. Reat. & 0,993 & $-17,2$ & 24,0 & 0,0 \\
\hline 12 & Carga & 0,934 & $-18,6$ & $-12,3$ & $-8,3$ \\
\hline 13 & C. Reat. & 0,969 & $-18,6$ & 0,0 & 24,0 \\
\hline 14 & Carga & 0,916 & $-19,9$ & $-6,8$ & $-1,8$ \\
\hline 15 & Carga & 0,911 & $-20,0$ & $-9,0$ & $-2,8$ \\
\hline 16 & Carga & 0,922 & $-19,4$ & $-3,9$ & $-2,0$ \\
\hline 17 & Carga & 0,918 & $-19,7$ & $-9,9$ & $-6,4$ \\
\hline 18 & Carga & 0,901 & $-20,8$ & $-3,5$ & $-1,1$ \\
\hline 19 & Carga & 0,899 & $-21,0$ & $-10,4$ & $-3,7$ \\
\hline 20 & Carga & 0,904 & $-20,7$ & $-2,4$ & $-0,8$ \\
\hline 21 & Carga & 0,909 & $-20,1$ & $-19,2$ & $-12,3$ \\
\hline 22 & Carga & 0,910 & $-20,1$ & 0,0 & 0,0 \\
\hline 23 & Carga & 0,898 & $-20,4$ & $-3,5$ & $-1,8$ \\
\hline 24 & Carga & 0,892 & $-20,6$ & $-9,6$ & $-7,4$ \\
\hline 25 & Carga & 0,889 & $-19,9$ & 0,0 & 0,0 \\
\hline 26 & Carga & 0,867 & $-20,5$ & $-3,9$ & $-2,5$ \\
\hline 27 & Carga & 0,898 & $-19,1$ & 0,0 & 0,0 \\
\hline 28 & Carga & 0,971 & $-13,5$ & 0,0 & 0,0 \\
\hline 29 & Carga & 0,873 & $-20,9$ & $-2,6$ & $-1,0$ \\
\hline 30 & Carga & 0,858 & $-22,2$ & $-11,7$ & $-2,1$ \\
\hline
\end{tabular}

No primeiro teste realizado para o sistema de 30 teve-se como objetivo minimizar a injeção de potência reativa de todas as barras de carga. Os resultados obtidos para este teste são mostrados na Tabela 14. Pode-se observar que foram alocados reativos em 11 barras diferentes, somando um total de 39,67 MVAr. Para os 
reativos alocados, apresentados na Tabela 14, as tensões atingiram níveis mais adequados.

Tabela 14 - Resultado do sistema AEP - 30 barras para a alocação reativa nas barras de carga do sistema

\begin{tabular}{|c|c|c|c|c|c|c|}
\hline \multirow[t]{2}{*}{ Barra } & \multirow[t]{2}{*}{ Tipo } & \multicolumn{2}{|c|}{ Tensão } & \multicolumn{2}{|c|}{ Potência } & \multirow{2}{*}{$\begin{array}{c}Q_{\text {sh }} \\
\text { Alocado } \\
\text { (MVAr) }\end{array}$} \\
\hline & & $\begin{array}{c}\mathrm{V} \\
\text { (p.u.) }\end{array}$ & $\begin{array}{c}\theta \\
\text { (graus) }\end{array}$ & $\begin{array}{c}\mathrm{P} \\
(\mathrm{MW})\end{array}$ & $\begin{array}{c}\mathrm{Q} \\
\text { (MVAt) }\end{array}$ & \\
\hline 1 & Slack & 1,053 & 0,0 & 289,5 & $-14,0$ & - \\
\hline 2 & Gera. & 1,032 & $-6,2$ & 20,1 & 36,7 & - \\
\hline 3 & Carga & 1,017 & $-9,1$ & $-2,6$ & $-1,3$ & - \\
\hline 4 & Carga & 1,010 & $-11,0$ & $-8,4$ & $-1,8$ & - \\
\hline 5 & C. Reat. & 0,994 & $-16,2$ & $-103,6$ & 19,0 & - \\
\hline 6 & Carga & 1,001 & $-12,9$ & 0,0 & 0,0 & - \\
\hline 7 & Carga & 0,989 & $-14,8$ & $-25,1$ & $-12,0$ & - \\
\hline 8 & C. Reat. & 1,000 & $-13,7$ & $-33,0$ & 7,0 & - \\
\hline 9 & Carga & 1,058 & $-16,4$ & 0,0 & 0,0 & - \\
\hline 10 & Carga & 1,056 & $-18,3$ & $-6,4$ & $-2,2$ & - \\
\hline 11 & C. Reat. & 1,072 & $-16,4$ & 0,0 & 7,0 & - \\
\hline 12 & Carga & 1,033 & $-17,2$ & $-12,3$ & $-8,3$ & - \\
\hline 13 & C. Reat. & 1,057 & $-17,2$ & 0,0 & 18,3 & - \\
\hline 14 & Carga & 1,030 & $-18,4$ & $-6,8$ & 1,2 & 3,0 \\
\hline 15 & Carga & 1,031 & $-18,6$ & $-9,0$ & $-2,8$ & - \\
\hline 16 & Carga & 1,036 & $-18,0$ & $-3,9$ & $-0,7$ & 1,33 \\
\hline 17 & Carga & 1,044 & $-18,4$ & $-9,9$ & $-6,0$ & 0,35 \\
\hline 18 & Carga & 1,034 & $-19,5$ & $-3,5$ & 1,1 & 2,19 \\
\hline 19 & Carga & 1,038 & $-19,7$ & $-10,4$ & 2,1 & 5,82 \\
\hline 20 & Carga & 1,041 & $-19,4$ & $-2,4$ & 0,8 & - \\
\hline 21 & Carga & 1,047 & $-18,9$ & $-19,2$ & $-6,7$ & 5,62 \\
\hline 22 & Carga & 1,047 & $-18,8$ & 0,0 & 0,0 & - \\
\hline 23 & Carga & 1,041 & $-19,4$ & $-3,5$ & 6,8 & 8,60 \\
\hline 24 & Carga & 1,042 & $-19,5$ & $-9,6$ & $-4,2$ & 3,22 \\
\hline 25 & Carga & 1,049 & $-18,9$ & 0,0 & 0,0 & - \\
\hline 26 & Carga & 1,041 & $-19,8$ & $-3,9$ & 0,3 & 2,81 \\
\hline 27 & Carga & 1,058 & $-18,2$ & 0,0 & 0,0 & - \\
\hline 28 & Carga & 0,999 & $-13,7$ & 0,0 & 0,0 & - \\
\hline 29 & Carga & 1,048 & $-19,8$ & $-2,6$ & $-0,3$ & 0,69 \\
\hline 30 & Carga & 1,046 & $-20,9$ & $-11,7$ & 3,9 & 6,04 \\
\hline
\end{tabular}

Em seguida, foram feitos novos testes seguindo a abordagem proposta. A partir de uma análise dos multiplicadores de Lagrange, determinaram-se as barras com maior sensibilidade à variação de potência reativa do sistema. A partir da determinação destas barras utilizou-se a programação linear para quantificar a potência reativa a ser alocada. A solução final para este teste é apresentada na Tabela 15. 
Tabela 15 - Resultado do sistema AEP - 30 barras para a alocação reativa nas barras determinadas pela abordagem proposta

\begin{tabular}{|c|c|c|c|c|c|c|}
\hline Barra & \multirow{2}{*}{ Tipo } & \multicolumn{2}{|c|}{ Tensão } & \multicolumn{2}{c|}{ Potência } & $\begin{array}{c}\text { Qsh } \\
\text { Alocado } \\
\text { (MVAr) }\end{array}$ \\
\cline { 3 - 6 } & & $\begin{array}{c}\mathrm{V} \\
\text { (p.u.) }\end{array}$ & $\begin{array}{c}\theta \\
\text { (graus) }\end{array}$ & $\begin{array}{c}\mathrm{P} \\
\text { (MW) }\end{array}$ & $\begin{array}{c}\text { Q } \\
\text { (MVAr) }\end{array}$ & - \\
\hline 1 & Slack & 1,053 & 0,0 & 289,9 & $-12,0$ & - \\
\hline 2 & Gera. & 1,031 & $-6,2$ & 20,1 & 36,0 & - \\
\hline 3 & Carga & 1,016 & $-9,1$ & $-2,6$ & $-1,3$ & - \\
\hline 4 & Carga & 1,009 & $-11,0$ & $-8,4$ & $-1,8$ & - \\
\hline 5 & C. Reat. & 0,992 & $-16,2$ & $-103,6$ & 19,0 & - \\
\hline 6 & Carga & 0,999 & $-12,9$ & 0,0 & 0,0 & - \\
\hline 7 & Carga & 0,987 & $-14,9$ & $-25,1$ & $-12,0$ & - \\
\hline 8 & C. Reat. & 0,998 & $-13,8$ & $-33,0$ & 7,0 & - \\
\hline 9 & Carga & 1,053 & $-16,5$ & 0,0 & 0,0 & - \\
\hline 10 & Carga & 1,045 & $-18,4$ & $-6,4$ & $-2,2$ & - \\
\hline 11 & C. Reat. & 1,072 & $-16,5$ & 0,0 & 9,8 & - \\
\hline 12 & Carga & 1,017 & $-17,3$ & $-12,3$ & $-8,3$ & - \\
\hline 13 & C. Reat. & 1,049 & $-17,3$ & 0,0 & 24,0 & - \\
\hline 14 & Carga & 1,030 & $-18,4$ & $-6,8$ & 1,2 & - \\
\hline 15 & Carga & 1,013 & $-18,7$ & $-9,0$ & $-2,8$ & - \\
\hline 16 & Carga & 1,021 & $-18,1$ & $-3,9$ & $-2,0$ & - \\
\hline 17 & Carga & 1,032 & $-18,5$ & $-9,9$ & $-6,4$ & - \\
\hline 18 & Carga & 1,025 & $-19,7$ & $-3,5$ & $-1,1$ & - \\
\hline 19 & Carga & 1,037 & $-20,1$ & $-10,4$ & 12,2 & 15,87 \\
\hline 20 & Carga & 1,038 & $-19,8$ & $-2,4$ & $-0,8$ & - \\
\hline 21 & Carga & 1,033 & $-18,9$ & $-19,2$ & $-12,3$ & - \\
\hline 22 & Carga & 1,034 & $-18,9$ & 0,0 & 0,0 & - \\
\hline 23 & Carga & 1,014 & $-19,1$ & $-3,5$ & 1,8 & - \\
\hline 24 & Carga & 1,025 & $-19,4$ & $-9,6$ & $-7,4$ & - \\
\hline 25 & Carga & 1,052 & $-19,2$ & 0,0 & 0,0 & - \\
\hline 26 & Carga & 1,059 & $-20,6$ & $-3,9$ & 4,9 & 7,38 \\
\hline 27 & Carga & 1,063 & $-18,4$ & 0,0 & 0,0 & - \\
\hline 28 & Carga & 0,998 & $-13,8$ & 0,0 & 0,0 & - \\
\hline 29 & Carga & 1,057 & $-20,1$ & $-2,6$ & $-1,0$ & - \\
\hline 30 & Carga & 1,061 & $-21,4$ & $-11,7$ & 7,4 & 9,51 \\
\hline PERDAS = 22,2MW & & & & \\
\hline & & & & & & \\
\hline
\end{tabular}

Observa-se que foram alocados reativos em 3 diferentes barras somando um total de 32,76 MVAr. Para os reativos alocados, apresentados na Tabela 15, as tensões atingiram níveis mais adequados. 


\section{4) Sistema IEEE - 118 barras}

O sistema IEEE - 118 barras, utilizado para realização dos testes, é apresentado na Figura 5. O sistema apresentado possui as seguintes características:

- 1 barra de referência;

- 51 barras de controle de reativos;

- 66 barras de carga;

- 169 linhas de transmissão;

- 9 transformadores com tap variável; 


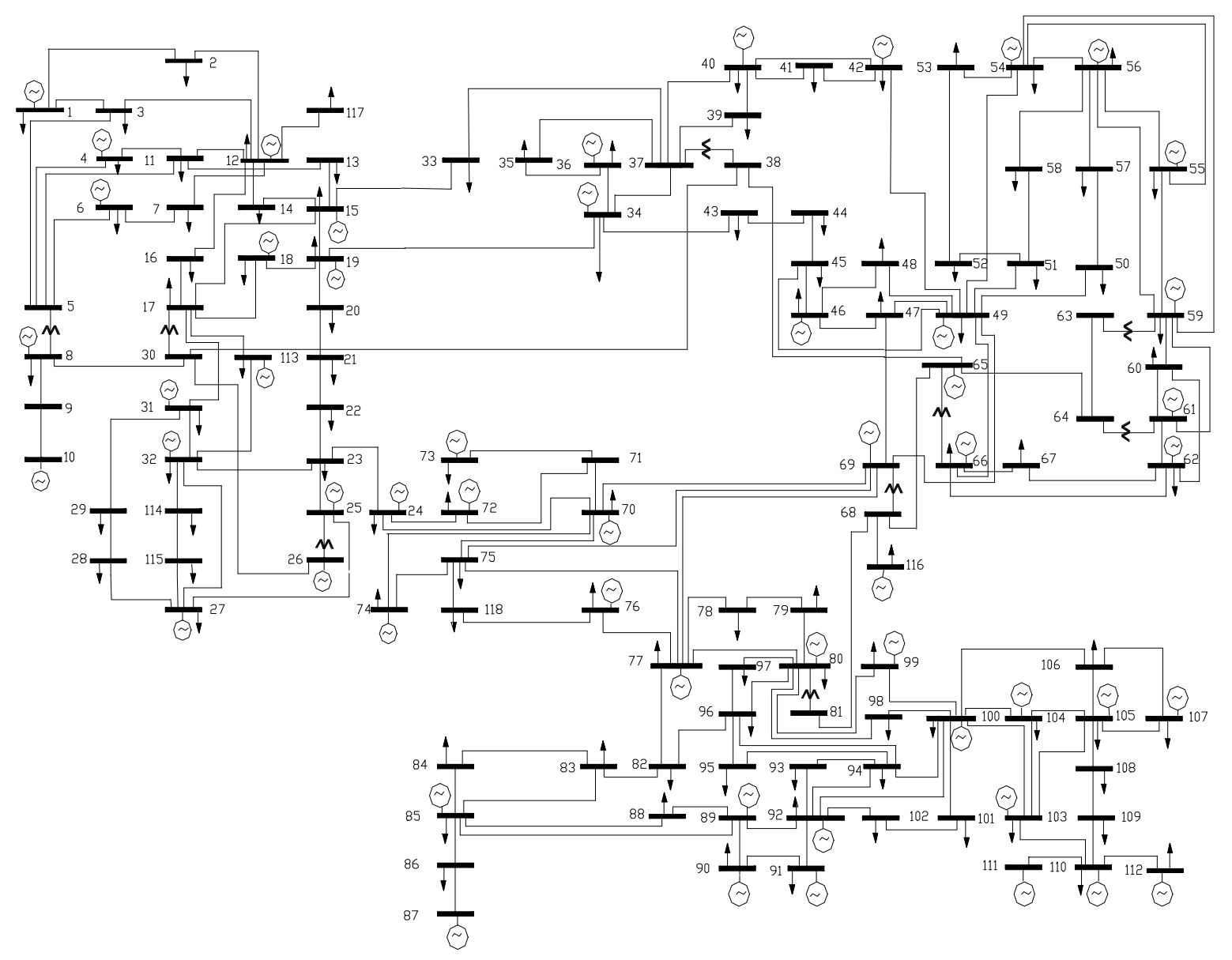

Figura 5- Sistema IEEE-118 barras

O estado final do sistema IEEE - 118 barras representado pela Figura 5 apresentou valores de tensões fora de seus limites. A tensão mínima foi apresentada pela Barra 112 alcançando o valor de 0,917 p.u., e a tensão máxima foi apresentada pela Barra 116 alcançando o valor de 1,001 p.u.. Mediante o valor mínimo de tensão apresentado pelo sistema, torna-se necessária à alocação de reativos.

No primeiro teste realizado para o sistema de 118 barras, a função objetivo do problema de programação linear foi minimizar a injeção de potência reativa de todas as barras de carga. Resolvendo o problema de programação linear, foi obtida uma quantidade de reativos, a ser alocada em 6 barras diferentes, com um total de 85,84 
MVAr, como apresentado pela Tabela 16. As tensões mostraram-se todas dentro de seus limites.

Tabela 16 - Resultado do sistema IEEE-118 barras para a alocação reativa nas barras de carga do sistema

\begin{tabular}{|c|c|c|c|c|c|c|}
\hline Barra & \multirow{2}{*}{ Tipo } & \multicolumn{2}{|c|}{ Tensão } & \multicolumn{2}{c|}{ Potência Gerada } & \multirow{2}{*}{$\begin{array}{c}\text { Qsh } \\
\text { Alocado } \\
\text { (MVAr) }\end{array}$} \\
\hline 21 & Carga & 0,953 & $-2,6$ & 0,0 & 5,20 & 5,20 \\
\hline 44 & Carga & 0,952 & $-3,9$ & 0,0 & 5,60 & 5,57 \\
\hline 95 & Carga & 0,950 & 15,1 & 0,0 & 19,00 & 19,01 \\
\hline 106 & Carga & 0,970 & 2,7 & 0,0 & 17,2 & 17,21 \\
\hline 108 & Carga & 0,976 & 1,3 & 0,0 & 3,00 & 3,02 \\
\hline 109 & Carga & 0,978 & 0,6 & 0,0 & 35,8 & 35,83 \\
\hline
\end{tabular}

Novos testes, seguindo a abordagem proposta, foram realizados. A partir de uma análise aos multiplicadores de Lagrange determinaram-se as barras com maior sensibilidade à variação de potência reativa do sistema. A partir da determinação destas barras, utilizou-se a programação linear para quantificar a potência reativa a ser alocada. Os resultados determinados pela abordagem proposta são apresentados pela Tabela 17.

Tabela 17 - Resultado do sistema IEEE-118 barras para a alocação reativa nas barras determinadas pela abordagem proposta

\begin{tabular}{|c|c|c|c|c|c|c|}
\hline \multirow[t]{2}{*}{ Barra } & \multirow[t]{2}{*}{ Tipo } & \multicolumn{2}{|c|}{ Tensão } & \multicolumn{2}{|c|}{ Potência Gerada } & \multirow{2}{*}{$\begin{array}{c}\mathrm{Q}_{\mathrm{sh}} \\
\text { Alocado } \\
\text { (MVAr) }\end{array}$} \\
\hline & & $\begin{array}{c}\mathrm{V} \\
\text { (p.u.) }\end{array}$ & $\begin{array}{c}\theta \\
\text { (graus) }\end{array}$ & $\begin{array}{c}\mathrm{P} \\
(\mathrm{MW})\end{array}$ & $\begin{array}{c}\mathrm{Q} \\
\text { (MVAr) }\end{array}$ & \\
\hline 21 & Carga & 0,952 & $-2,6$ & 0,0 & 5,20 & 5,20 \\
\hline 45 & Carga & 0,951 & $-1,5$ & 0,0 & 3,60 & 3,64 \\
\hline 95 & Carga & 0.950 & 15,1 & 0,0 & 19,01 & 19,01 \\
\hline 106 & Carga & 0,970 & 2,7 & 0,0 & 17,21 & 17,21 \\
\hline 109 & Carga & 0,977 & 0,6 & 0,0 & 38,0 & 37,95 \\
\hline
\end{tabular}


A solução final para este teste alocou reativos em 5 barras diferentes, somando um total de 83,01 MVAr. Para os reativos alocados, as tensões atingiram níveis mais adequados apresentando para a Barra 44 a menor tensão com valor de 0,946 p.u.. O limite superior não foi violado e apresentou para a Barra 5 o maior nível de tensão no valor de 1,003 p.u.. 


\section{CONCLUSÃO}

A abordagem proposta neste trabalho visa a contribuir no planejamento de reativos em sistemas elétricos de potência, utilizando métodos de otimização com o intuito de determinar a localização e a magnitude das fontes reativas assegurando, assim, a operação do sistema de energia elétrica de forma que atenda os limites físicos e de segurança pré-estabelecidos.

Para este trabalho, propõe-se o emprego do método primal-dual barreira logarítmica, como método de otimização não linear, para determinar as barras do sistema em que serão alocados os reativos via multiplicadores de Lagrange. A partir da determinação destas barras, aplica-se o método Simplex com a finalidade de determinar a quantidade de reativos a serem alocados. Para a implementação do método primal-dual barreira logarítmica, foi utilizada a linguagem de programação FORTRAN. Para a implementação do método Simplex, foi empregado o software LINDO API, que utiliza como interface o software MATLAB.

Os resultados obtidos pelos testes mostraram-se coerentes com a teoria e produziram excelentes respostas, levando-nos a concluir que o método proposto para o planejamento de reativos apresenta boas perspectivas para a melhoria das condições operacionais dos sistemas elétricos de potência. 
Para os testes realizados, a solução do problema de programação linear alocou reativos elevando os níveis de tensão para valores bem próximos do limite mínimo desejado, o qual não foi atingido em alguns casos. Nestes casos, esse limite não foi atingido devido ao fato de o fluxo de carga ser um problema não linear e de a abordagem apresentada utilizar uma aproximação linear para quantificar a potência reativa a ser alocada. 
ABDEL-SALAM, T.S.; CHIKHANI, A.Y.; HACKAM, R. (1994). A New Technique for Loss Reduction Using Compensating Capacitors Applied to Distribution Systems with Varying Load Condition. IEEE Trans. on Power Delivery, v.9, n.2, p. 819-827, April.

BAE, Y.G. (1978). Analytical Method of Capacitor Allocation on Distribution Primary Feeders. IEEE Trans. Power Apparatus and Systems, v.88, n.11, p. 1232-1238, July/August.

BOONE, G.; CHIANG, H.D. (1993). Optimal Capacitor Placement in Distribution Systems by Genetic Algorithm. Electrical Power and Energy Systems, v. 15, n.3, p. $155-162$.

CARPENTIER, J.L. (1962). Contribution a L'etude du Dispatching Economique. Bull-Soc. Fr. Elec. Ser. B3, p. 431-447.

CHAVES, F.S.; VALE, M. H. M. (2002). Controle de Tensão e Compensação Reativa - Procedimento Aplicado à Expansão de Sistemas Elétricos. XIV Congresso Brasileiro de Automática, Setembro.

CHIN, H.C. (1995). Optimal Shunt Capacitor Allocation by Fuzzy Dynamic Programming. Electrical Power Systems Research, v.35, p. 133-139. 
CHIANG, H.D.; WANG, J.C.; COCKINGS, O.; SHIN, H.D. (1990). Optimal Capacitor Placements in Distribution Systems. Part 1: A New Formulation and the Overall Problem. IEEE Trans. on Power Delivery, v.5, n.2, p. 634-642, April.

. Optimal Capacitor Placements in Distribution Systems. Part 2: Solution Algorithms and Numerical Results. IEEE Trans. on Power Delivery, v.5, n.2, p. 643-649, April.

CIVANLAR S.; GRAINGER, J.J. (1985). Volt/VAr control on Distribution Systems with Lateral Branches Using Shunt Capacitors and Voltage Regulators. Part 2: The Solution Method. IEEE Trans. Power Apparatus and Systems, v.104, n.11, p. 3284-3290, November.

. Volt/VAr control on Distribution Systems with Lateral Branches Using Shunt Capacitors and Voltage Regulators. Part 3: The Numerical Results. IEEE Trans. Power Apparatus and Systems, v.104, n.11, p. 3291-3297, November.

COOK, R.F. (1959). Analysis of Capacitor Application as Affected by Load Cycle. AIEE Trans., v.78, p.950-957, October.

DEEB, N.; SHAHIDEHPOUR, S.M. (1989). Economic Allocation of Reactive Power Supply in an Electric Power Network. ISCAS - IEEE, p.1859-1862.

DURAN, H. (1968). Optimum Number, Location and Size of Shunt Capacitors in Radial Distribution Feeders. IEEE Trans. Power Apparatus and Systems, v.87, n.9, p. 1769-1774, September.

EL-HAWARY, M. E.; MEKHAMER, S. F.; MANSOUR, M. M.; MOUSTAFA, M. A.; SOLIMAN, S. A. (2002). State of the Art in Optimal Capacitor Allocation for Reactive Power Compensation in Distribution Feeders. Proceedings of the 2002 Large Engineering Systems Conference on Power Engineering, p.61-75. 
ELRAZAZ, Z.S. (2001). Optimal Allocation of Reactors for Light Load Operation. IEE Proc. - Gener. Transm, Distrib., v.148, n.4, p.350-354, July.

FAWZI, T.H.; EL-SOBKI, S.M.; ABDEL-HALIM, M.A. (1983). New Approach for the Application of Shunt Capacitors to the Primary Distribution Feeders. IEEE Trans. Power Apparatus and Systems, v.102, n.1, p. 10-13, January.

FRITZSCHE, H. (1978). Programação Não-Linear: Análise e Métodos. São Paulo, Edgard Blücher Ltda.

GALlEGO, R.A.; MONTICELLI, A.J.; ROMERO, R. (2001). Optimal Capacitor Placement in Radial Distribution Networks. IEEE Trans. on Power Systems, v.16, n.4, p. 630-637, November.

GRAINGER, J.J.; LEE, S.H. (1981). Optimum Size and Location of Shunt Capacitors for Reduction of Losses on Distribution Feeders. IEEE Trans. Power Apparatus and Systems, v.100, n.3, p. 1105-1118, March.

GRAINGER, J.J.; CIVANLAR S. (1985). Volt/VAr control on Distribution Systems with Lateral Branches Using Shunt Capacitors and Voltage Regulators. Part 1: The Overall Problem. IEEE Trans. Power Apparatus and Systems, v.104, n.11, p. 3278-3283, November.

GRANVILLE, S. (1994). Optimal Reactive Dispatch through Interior Point Method. IEEE Trans. on Power Systems, v.9, n.1, p. 136-146, February.

GRUDININ, N. (1998). Reactive Power Optimization Using Successive Quadratic Programming Method. IEEE Trans. on Power Systems, v.13, n.4, p. 1219-1225, November. 
HSIAO, Y.T.; CHIEN, C.Y. (2001). Optimisation of Capacitor Allocation Using an Interactive Trade-off Method. IEE Proc. - Gener. Transm, Distrib., v.148, n.4, p.371-376, July.

IYER, S.R.; RAMACHANDRAN, K.; HARIHARAN, S. (1984). Optimal Reactive Power Allocation for Improved System Performance. IEEE Trans. Power Apparatus and Systems, v. PAS-103, n.6, p. 1509-1515, June.

KISHORE, A.; HILL, E.F. (1970). Static Optimization of Reactive Power Sources by Use of Sensitivity Parameters. IEEE Power Group, p.1166-1173, April.

LEE, K.Y.; YANG, F.F. (1998). Optimal Reactive Power Planning Using Evolutionary Algorithms: A Comparative Study for Evolutionary Programming, Evolutionary Strategy, Genetic Algorithm and Linear Programming. IEEE Trans. on Power Systems, v.13, n.1, p. 101-108, February.

MAMANDUR, K.R.C.; CHENOWETH, R.D. (1981). Optimal Control of Reactive Power Flow for Improvements in Voltage Profiles and for Real Power Loss Minimization. IEEE Trans. Power Apparatus and Systems, v. PAS-100, n.7, p. 3185-3194, July.

MANTOVANI, J.R.S. (1994). Um Modelo Heurístico para Solução do Problema de Planejamento de Reativos em Sistemas de Energia Elétrica. Tese (Doutorado) Faculdade de Engenharia Elétrica, Unicamp, Campinas, Dezembro.

MANTOVANI, J.R.S.; MODESTO, S.A.G.; GARCIA, A.V. (2001a). VAr Planning Using Genetic Algorithm and Linear Programming. . IEE Proc. - Gener. Transm, Distrib., v.148, n.3, p.257-262, May.

MANTOVANI, J.R.S.; SCUCUGLIA, J.W.; ROMERO,R.; GARCIA, A.V. (2001b). Planejamento de Fontes Reativas em Sistemas de Energia Elétrica Utilizando a 
Técnica de Decomposição de Benders e o Algoritmo de Branch-and-Bound. SBA Controle e Automação, v.12, n.2, p.131-140, Maio - Agosto.

MONTICELli, A. (1983). Fluxo de Carga em Redes de Distribuição de Energia Elétrica. São Paulo, Edgard Blücher Ltda.

NEAGLE, N.M.; SAMSON, D.R. (1956). Loss Reduction from Capacitors Installed on Primary Feeders. AIEE Trans., v.75, p.950-959, October.

NG, H.N.; SALAMA, M.M.A.; CHIKHANI, A.Y. (2000a). Capacitor Allocation by Approximate Reasoning: Fuzzy Capacitor Placement. IEEE Trans. on Power Delivery, v.13, n.1, p. 393-398, January.

NG, H.N.; SALAMA, M.M.A.; CHIKHANI, A.Y. (2000b). Classification of Capacitor Allocation Techniques. IEEE Trans. on Power Delivery, v.13, n.1, p. 387-392, January.

NOUREDDINE, A.H.; CHANDRASEKARAN, A. (1992). Linear Programming Approach to Loss Minimization and Capacitor Sizing and Placement. SSST/CSA 92, p.454-458.

QUINTANA, V.H.; GOMEZ, A.; MARTINEZ, J. L. (1995). Nonlinear Optimal Power Flows by Logarithmic-Barrier Primal-Dual Algorithm. IEEE NAPS Meeting.

RAMAlhete, M.; GUERREIRO, J.; MAGAlHÃES, A. (1984). Programação Linear. McGRAW-HILL, v.1.

REZANIA, E.; SHAHIDEHPOUR, S.M. (2001). Real Power Loss Minimization Using Interior Point Method. Electrical Power and Energy Systems, n.23, p.4556. 
SALAMA, M.M.A.; CHIKHANI, A.Y.; HACKAM, R. (1985). Control of Reactive Power in Distribution Systems with an End-load and Fixed Load Conditions. IEEE Trans. Power Apparatus and Systems, v. 104, n.4, p. 941-947, April.

SALAMA, M.M.A.; LAFRAMBOISE, J.R.P.R.; FERLAND, G.; CHIKHANI, A.Y. (1995). An Expert System For Reactive Power Control of a Distribution System. Part 2: System Implementation. IEEE Trans. Power Systems, v.10, n.3, p. 14331441, August.

SANTOSO, N.I.; TAN, O.T. (1990). Neural-net Based Real-time Control of Capacitors Installed on Distribution Systems. IEEE Trans. Power Delivery, v.5, n.1, p. 266-272, January.

SCHMILL, J.V. (1965). Optimum Size and Location of Shunt Capacitors on Distribution Feeders. IEEE Trans. Power Apparatus and Systems, v. 84, n.9, p. 825-832, September.

SOUSA, V. A. (2001). A Função Barreira Logarítmica Associada ao Método de Newton Modificado para a Resolução do Problema de Fluxo de Potência Ótimo. Dissertação (Mestrado) - Escola de Engenharia de São Carlos, Usp, São Carlos.

SOUZA, A. M. (1998). O Método Primal Dual Barreira Logarítmica Aplicado ao Problema de Fluxo de Carga Ótimo. Dissertação (Mestrado) - Escola de Engenharia de São Carlos, Usp, São Carlos.

ZADEH, L.A. (1965). Fuzzy Sets. Information and Control, v.8, p.338-353. 


\section{PROGRAMAÇÃO LINEAR - MÉTODO DO SIMPLEX}

A programação linear visa fundamentalmente encontrar a melhor solução para problemas que tenham seus modelos representados por expressões lineares. A tarefa da programação linear consiste na maximização ou minimização de uma função linear, sobre um conjunto poliédrico denominado de função objetivo, respeitando-se um sistema linear de igualdade ou desigualdade que recebem o nome de restrições do modelo ou região factível. As restrições representam normalmente limitações de recursos disponíveis ou, então, exigências e condições que devem ser cumpridas no problema. Essas restrições do modelo determinam uma região à qual dá-se o nome de conjunto das soluções viáveis ou admissíveis. O método é principalmente baseado na exploração dos pontos extremos do conjunto das soluções viáveis e nas direções do conjunto poliédrico definido no problema.

A melhor das soluções admissíveis, isto é, aquela que maximiza ou minimiza a função objetivo denomina-se solução ótima. O objetivo da programação linear consiste na determinação dessa solução ótima. Dois passos são fundamentais para a resolução de um problema de programação linear. O primeiro é a modelagem do problema, seguido do método de solução do modelo.

O modelo matemático que representa a programação linear é freqüentemente apresentado na forma: 
Maximizar(Minimizar) $z=c_{1} x_{1}+c_{2} x_{2}+\ldots+c_{N} x_{N}$

Sujeito a :

$$
\begin{array}{r}
a_{11} x_{1}+a_{12} x_{2}+\ldots+a_{1 N} x_{N} \leq b_{1} \\
a_{21} x_{I}+a_{22} x_{2}+\ldots+a_{2 N} x_{N} \leq b_{2} \\
\ldots \\
a_{M 1} x_{1}+a_{M 2} x_{2}+\ldots+a_{M N} x_{N} \leq b_{M} \\
x_{j} \geq 0, j=1,2, \ldots, N
\end{array}
$$

A função maximizar (minimizar), $z=c_{1} x_{1}+c_{2} x_{2}+\ldots+c_{N} x_{N}$, designa-se por função objetivo; as inequações (A.2) designam-se por restrições funcionais ou simplesmente restrições; as desigualdades (A.3) designam-se por condições de não negatividade. As variáveis $x_{j}$ são chamadas por variáveis principais, de decisão ou controláveis; as constantes $a_{i j}, b_{i}$ e $c_{j}$ são chamados de coeficientes técnicos, termos independentes e coeficientes da função objetivo, respectivamente, constituindo os parâmetros do modelo.

Uma forma de resolução de grande utilidade quando se inicia o estudo de programação linear é a representação gráfica dos problemas. O grande inconveniente destas representações é o fato de só serem possíveis quando não estão envolvidas mais de três variáveis, sendo incapazes de resolver problemas de maiores dimensões. Diante desse inconveniente, torna-se necessário utilizar um procedimento analítico suficientemente geral que não restrinja a dimensão do problema.

Como em geral é mais conveniente trabalhar com igualdades do que com desigualdades, o primeiro passo a cumprir com o objetivo de resolver um problema de programação linear consiste em converter as restrições funcionais de desigualdade em restrições equivalentes sob a forma de igualdade, obtendo-se assim um sistema de equações lineares. Esse processo é alcançado pela introdução no problema original de 
novas variáveis, igualmente não negativas, designadas por variáveis auxiliares. Diz-se então que se reduziu o problema original à forma estandardizada. Assim, a redução à forma estandardizada do problema de programação linear pode ser representada por:

$$
\begin{aligned}
& \text { Maximizar (Minimizar) } z=c_{1} x_{1}+c_{2} x_{2}+\ldots+c_{N} x_{N}+c_{N+1} x_{N+1}+\ldots+c_{N+M} x_{N+M} \\
& \text { Sujeito a: } \quad a_{11} x_{1}+a_{12} x_{2}+\ldots+a_{1 N} x_{N}+a_{1, N+1} x_{N+1} \quad=b_{1} \\
& a_{21} x_{1}+a_{22} x_{2}+\ldots+a_{2 N} x_{N} \quad+x_{N+2} \quad=b_{2} \\
& a_{M 1} x_{1}+a_{M 2} x_{2}+\ldots+a_{M N} x_{N} \quad+a_{M, N+M} x_{N+M}=b_{M} \\
& x_{j} \geq 0, j=1,2, \ldots, N, N+1, \ldots, N+M
\end{aligned}
$$

$\mathrm{O}$ conjunto das soluções que satisfazem as restrições funcionais (A.5) e as restrições de não negatividade (A.6), $K$, de um problema de programação linear é um conjunto convexo fechado. Uma função linear sobre esse conjunto convexo, $K$, atinge o ótimo em um ponto extremo de $K$. O conjunto de pontos extremos de $K$ é finito e para este conjunto existe pelo menos um ponto extremo de $K$ que otimiza a função objetivo.

Um conjunto de $m$ variáveis tais que a matriz dos coeficientes respectivos no sistema de equações lineares (A.5) seja não singular (isto é, cujo determinante seja não nulo) chama-se base do sistema (A.5) e as $m$ variáveis por variáveis básicas. As restantes $n-m$ variáveis são designadas por variáveis não básicas. Atribuindo a estas variáveis o valor zero, as variáveis básicas serão então determinadas, designando-se a solução assim obtida por solução básica. Se as variáveis básicas forem não negativas, designa-se por solução básica admissível.

O problema de programação linear, no caso de $K$ ser um poliedro convexo, é então teoricamente resolvido, pois a investigação exaustiva no conjunto das soluções 
básicas admissíveis é suficiente. Contudo, na generalidade dos casos de interesse prático, tal procedimento constitui tarefa impraticável. Além disso, no caso de $K$ ser não limitado, o exame exaustivo dos seus pontos extremos não detecta se o problema tem solução não limitada. Torna-se então necessário apresentar um procedimento que permita resolver qualquer problema de programação linear.

É natural que o processo de resolução mais generalizado seja um método iterativo que procura examinar o menor número possível de soluções básicas admissíveis. Um método que permite resolver este tipo de problema é designado por Método do Simplex, proposto em 1947 por George B. Dantzig.

O Método do Simplex é um procedimento sistemático para solucionar um problema de programação linear por mover de um ponto extremo a um ponto extremo com um melhor valor de função objetivo. Esse processo continua até que um ponto extremo ótimo é alcançado. Para um melhor entendimento do Método do Simplex, um exemplo pode ser explorado em RAMALHETE (1984). 


\section{BANCO DE DADOS DOS SISTEMAS ELÉTRICOS}

- Banco de dados do sistema de 6 barras

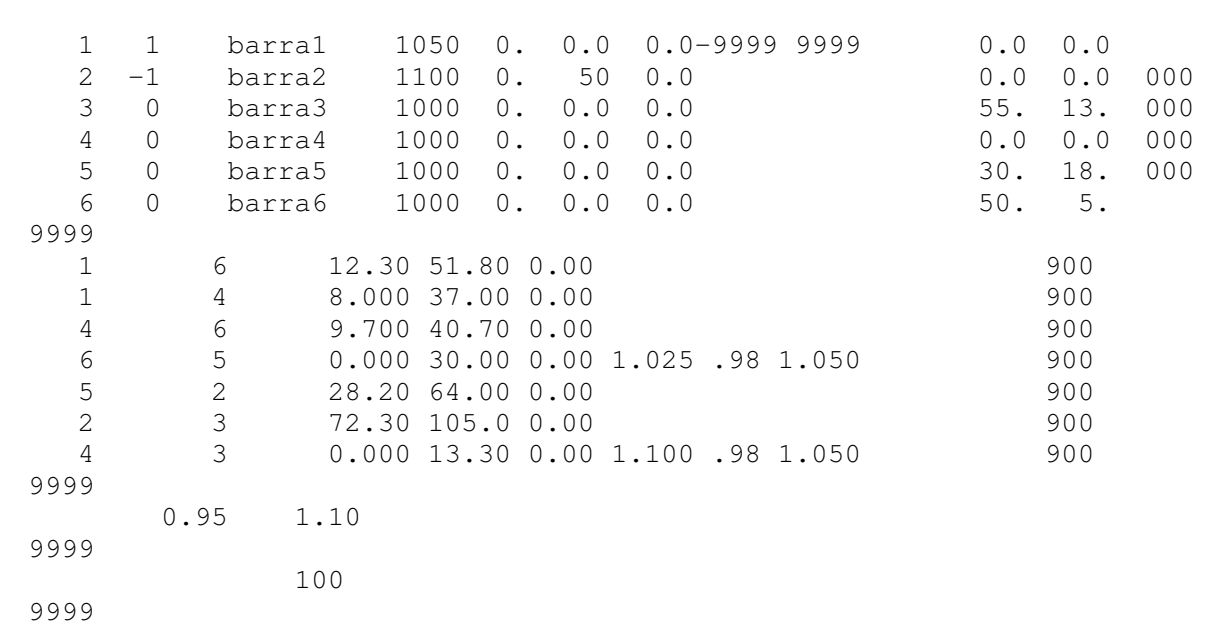

- Banco de dados do sistema de 8 barras

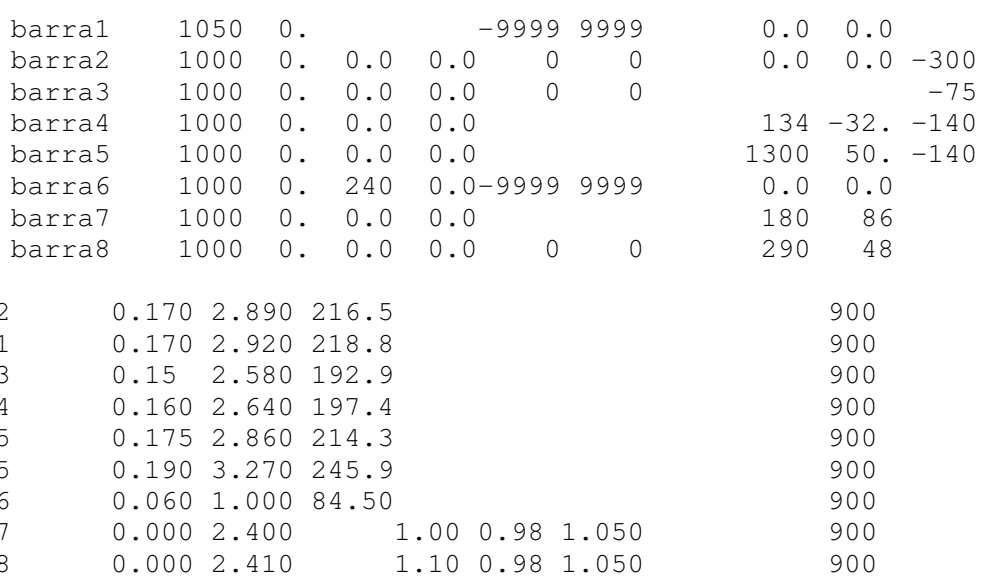




$\begin{array}{rrrrrr}22 & 24 & 11.50 & 17.90 & & 9000 \\ 23 & 24 & 13.20 & 27.00 & & 9000 \\ 24 & 25 & 18.85 & 32.92 & & 9000 \\ 25 & 26 & 25.44 & 38.00 & & 9000 \\ 25 & 27 & 10.93 & 20.87 & & 9000 \\ 28 & 27 & 0.0 & 39.60 & .9580 .9501 .05 & 9000 \\ 27 & 29 & 21.98 & 41.53 & & 9000 \\ 27 & 30 & 32.02 & 60.27 & & 9000 \\ 29 & 30 & 23.99 & 45.33 & & 9000 \\ 8 & 28 & 6.36 & 20.00 & 2.14 & 9000 \\ 6 & 28 & 1.69 & 5.99 & 0.65 & 9000 \\ 9999 & & & & & \\ 9999 & 0.95 & 1.1 & & & \\ 9999 & & & & & \end{array}$

\section{Banco de dados do sistema IEEE-118 barras}

\begin{tabular}{|c|c|c|}
\hline 12 & 2 BARRA12 & 1000 \\
\hline 2 & 0 BARRA2 & 1000 \\
\hline 3 & 0 BARRA3 & 1000 \\
\hline 4 & 1 BARRA4 & 1000 \\
\hline 5 & 0 BARRA5 & 1000 \\
\hline 6 & 1 BARRA 6 & 1000 \\
\hline 7 & 0 BARRA 7 & 1000 \\
\hline 8 & 1 BARRA 8 & 1000 \\
\hline 9 & 0 BARRA 9 & 1000 \\
\hline 10 & 1 BARRA 10 & 1000 \\
\hline 11 & 0 BARRA1 1 & 1000 \\
\hline 1 & 1 BARRA1 & 1000 \\
\hline 13 & 0 BARRA 13 & 1000 \\
\hline 14 & 0 BARRA14 & 1000 \\
\hline 15 & 1 BARRA15 & 1000 \\
\hline 16 & 0 BARRA1 6 & 1000 \\
\hline 17 & 0 BARRA17 & 1000 \\
\hline 18 & 1 BARRA18 & 1000 \\
\hline 19 & 1 BARRA19 & 1000 \\
\hline 20 & 0 BARRA20 & 1000 \\
\hline 21 & 0 BARRA2 1 & 1000 \\
\hline 22 & 0 BARRA22 & 1000 \\
\hline 23 & 0 BARRA23 & 1000 \\
\hline 24 & 0 BARRA2 4 & 1000 \\
\hline 25 & 1 BARRA2 5 & 1000 \\
\hline 26 & 1 BARRA2 6 & 1000 \\
\hline 27 & 1 BARRA27 & 1000 \\
\hline 28 & 0 BARRA28 & 1000 \\
\hline 29 & 0 BARRA2 9 & 1000 \\
\hline 30 & 0 BARRA 30 & 1000 \\
\hline 31 & 1 BARRA31 & 1000 \\
\hline 32 & 1 BARRA32 & 1000 \\
\hline 33 & 0 BARRA33 & 1000 \\
\hline 34 & 0 BARRA34 & 1000 \\
\hline 35 & 0 BARRA35 & 1000 \\
\hline 36 & 1 BARRA3 6 & 1000 \\
\hline 37 & 0 BARRA37 & 1000 \\
\hline 38 & 0 BARRA38 & 1000 \\
\hline 39 & 0 BARRA39 & 1000 \\
\hline 40 & 1 BARRA 40 & 1000 \\
\hline 41 & 0 BARRA4 1 & 1000 \\
\hline 42 & 1 BARRA 42 & 1000 \\
\hline 43 & 0 BARRA43 & 1000 \\
\hline 44 & 0 BARRA 44 & 1000 \\
\hline 45 & 0 BARRA4 5 & 1000 \\
\hline 46 & 1 BARRA4 6 & 1000 \\
\hline 47 & 0 BARRA 47 & 1000 \\
\hline 48 & 0 BARRA 48 & 1000 \\
\hline
\end{tabular}

\begin{tabular}{|c|c|c|c|c|}
\hline-9999 & 9999 & .0 & .0 & \\
\hline & & 30.0 & 13.5 & \\
\hline & & 58.5 & 15.0 & \\
\hline-50 . & 50. & 58.5 & 18.0 & \\
\hline & & .0 & & 40. \\
\hline-50 . & 50. & 78.0 & 33.0 & \\
\hline & & 28.5 & 3.0 & \\
\hline-50 . & 50. & 42.0 & 0.0 & \\
\hline & & .0 & .0 & \\
\hline-250. & 250 . & -675 & .0 & \\
\hline & & 105 & 34.5 & \\
\hline-50 . & 50. & 76.5 & 40.5 & \\
\hline & & 51.0 & 24.0 & \\
\hline & & 21.0 & 1.5 & \\
\hline-50 . & 50. & 135. & 45.0 & \\
\hline & & 37.5 & 15.0 & \\
\hline & & 16.5 & 4.5 & \\
\hline-50 . & 50. & 90.0 & 51.0 & \\
\hline-50 . & 115 . & 67.5 & 37.5 & \\
\hline & & 27.0 & 4.5 & \\
\hline & & 21.0 & 12.0 & \\
\hline & & 15.0 & 7.5 & \\
\hline & & 10.5 & 4.5 & \\
\hline & & 19.5 & .0 & \\
\hline-250 & 250 . & -330 & .0 & \\
\hline-250 & 250 . & -471 & .0 & \\
\hline-50 & 50. & 107. & 19.5 & \\
\hline & & 25.5 & 10.5 & \\
\hline & & 36.0 & 6.0 & \\
\hline & & 0.0 & 0.0 & \\
\hline-50 . & 50. & 54.0 & 40.5 & \\
\hline-50 . & 100. & 88.5 & 34.5 & \\
\hline & & 34.5 & 13.5 & \\
\hline & & 88.5 & 39.01 & 3.99 \\
\hline & & 49.5 & 13.5 & \\
\hline-50 . & 50. & 46.5 & 25.5 & \\
\hline & & .0 & .0 & 25. \\
\hline & & .0 & .0 & \\
\hline & & 40.5 & 16.5 & \\
\hline-5 & 150. & 99.0 & 34.5 & \\
\hline & & 55.5 & 15.0 & \\
\hline-50 . & 50. & 144 . & 34.5 & \\
\hline & & 27.0 & 10.5 & \\
\hline & & 24.0 & 12.0 & 10. \\
\hline & & 79.5 & 33.0 & 10. \\
\hline- & 50. & 13.5 & 15.0 & 10. \\
\hline & & 51.0 & .0 & \\
\hline & & 30.0 & 16.51 & 5.0 \\
\hline
\end{tabular}




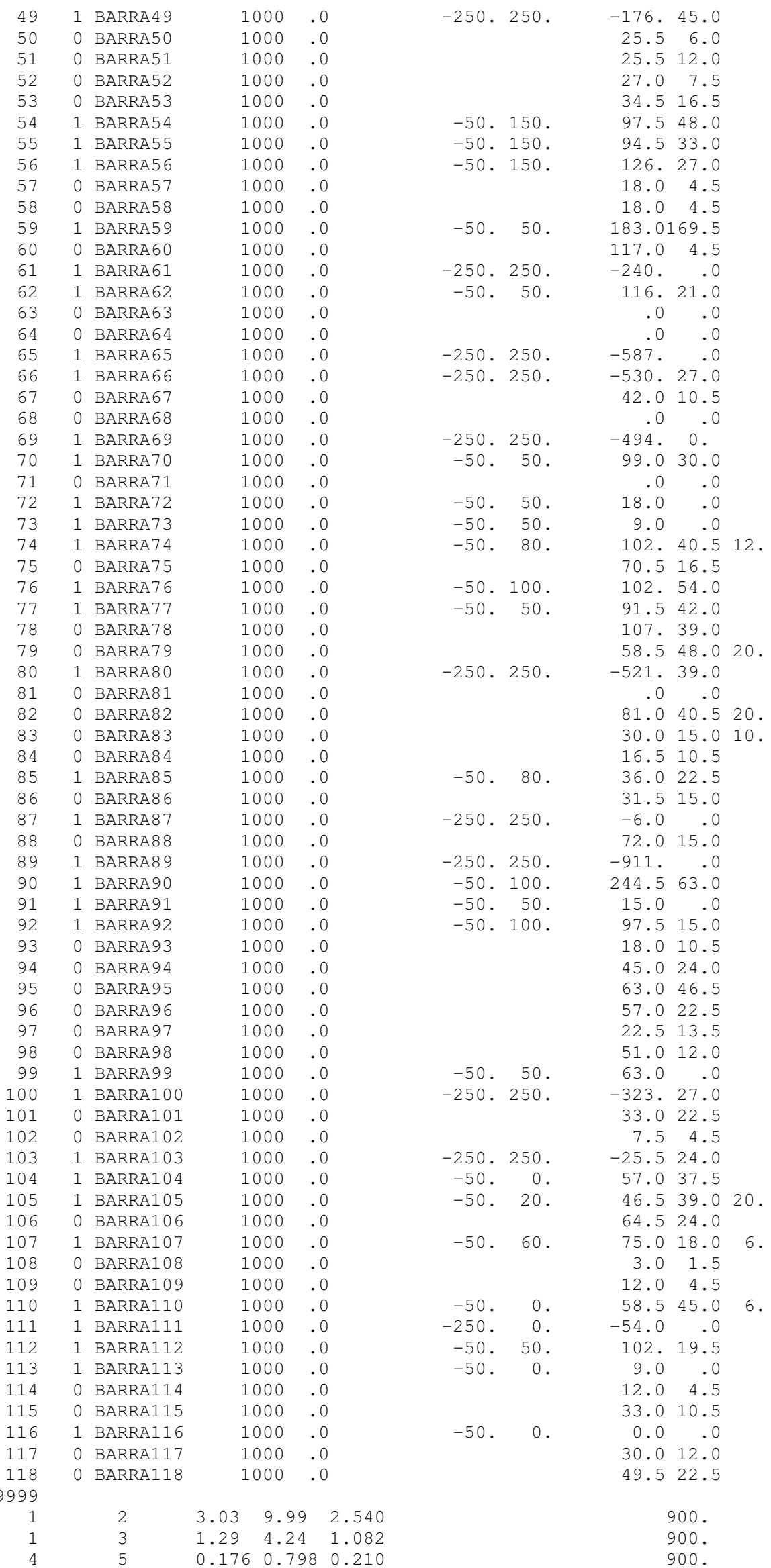


$\begin{array}{llll}2.41 & 10.80 & 2.840 & 900 .\end{array}$

$\begin{array}{llll}1.19 & 5.40 & 1.426 & 900 .\end{array}$

$\begin{array}{llll}0.2443 .05116 .62 & 900 .\end{array}$

$\begin{array}{lllllll}0.00 & 2.67 & 0.000 & 1.0 & .95 & 1.05 & 900 .\end{array}$

$\begin{array}{llll}0.258 & 3.22 & 123.00 & 900 .\end{array}$

$\begin{array}{llll}2.09 & 6.88 & 1.748 & 900 .\end{array}$

$\begin{array}{llll}2.03 & 6.82 & 1.738 & 900 .\end{array}$

$\begin{array}{llll}0.595 & 1.96 & 0.502 & 900 .\end{array}$

$\begin{array}{llll}1.87 & 6.16 & 1.572 & 900 .\end{array}$

$\begin{array}{llll}4.84 & 16.00 & 4.060 & 900 .\end{array}$

$\begin{array}{llll}0.862 & 3.40 & 0.874 & 900 .\end{array}$

$\begin{array}{llll}2.225 & 7.31 & 1.876 & 900 .\end{array}$

$\begin{array}{llll}2.15 & 7.07 & 1.816 & 900 .\end{array}$

$\begin{array}{llll}7.44 & 24.44 & 6.268 & 900 .\end{array}$

$\begin{array}{llll}5.95 & 19.50 & 5.020 & 900 .\end{array}$

$\begin{array}{llll}2.12 & 8.34 & 2.140 & 900 .\end{array}$

$\begin{array}{llll}1.32 & 4.37 & 4.440 & 900 .\end{array}$

$\begin{array}{rrrr}4.54 & 18.01 & 4.660 & 900 . \\ 1.23 & 5.05 & 1.298 & 900 .\end{array}$

$\begin{array}{llll}1.119 & 4.93 & 1.142 & 900 .\end{array}$

$\begin{array}{llll}2.52 & 11.70 & 2.980 & 900 .\end{array}$

$\begin{array}{llll}1.20 & 3.94 & 1.010 & 900 .\end{array}$

$\begin{array}{llll}1.83 & 8.49 & 2.160 & 900 .\end{array}$

$\begin{array}{llll}2.09 & 9.70 & 2.460 & 900 .\end{array}$

$\begin{array}{llll}3.42 & 15.90 & 4.040 & 900 .\end{array}$

$\begin{array}{llll}1.35 & 4.92 & 4.980 & 900 .\end{array}$

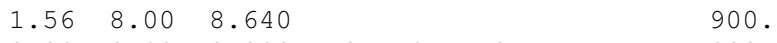

$\begin{array}{lllllll}0.00 & 3.82 & 0.000 & 1.0 & .95 & 1.05 & 900 .\end{array}$

$\begin{array}{llll}3.18 & 16.3017 .640 & 900 .\end{array}$

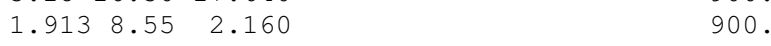

$\begin{array}{llll}2.37 & 9.43 & 2.380 & 900 .\end{array}$

$\begin{array}{lllllll}0.00 & 3.88 & 0.000 & 1.0 & .95 & 1.05 & 900 .\end{array}$

$\begin{array}{llll}.431 & 5.0451 .400 & 900 \text {. }\end{array}$

$\begin{array}{llll}.799 & 8.6091 .800 & 900 .\end{array}$

$\begin{array}{rrrr}4.74 & 15.63 & 3.990 & 900 .\end{array}$

$\begin{array}{llll}1.08 & 3.31 & 0.830 & 900 .\end{array}$

$\begin{array}{llll}3.17 & 11.53 & 11.730 & 900 .\end{array}$

$\begin{array}{llll}2.98 & 9.85 & 2.510 & 900 .\end{array}$

$\begin{array}{llll}2.29 & 7.55 & 1.926 & 900 .\end{array}$

$\begin{array}{llll}3.80 & 12.44 & 3.194 & 900 .\end{array}$

$\begin{array}{llll}7.52 & 24.70 & 6.320 & 900 .\end{array}$

$\begin{array}{rrrr}.224 & 1.02 & .268 & 900 . \\ 1.10 & 4.97 & 1.318 & 900 .\end{array}$

$\begin{array}{llll}4.15 & 14.20 & 3.660 & 900 .\end{array}$

$\begin{array}{llll}.871 & 2.68 & 0.568 & 900 .\end{array}$

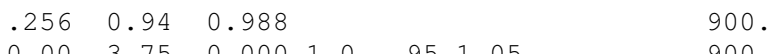

$\begin{array}{lllllll}0.00 & 3.75 & 0.000 & 1.0 & .95 & 1.05 & 900 .\end{array}$

$\begin{array}{llll}3.21 & 10.60 & 2.700 & 900 .\end{array}$

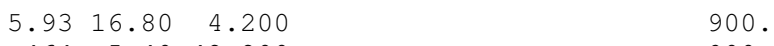

$\begin{array}{llll}.464 & 5.40 & 42.200 & 900 .\end{array}$

$\begin{array}{llll}1.84 & 6.05 & 1.552 & 900 .\end{array}$

$\begin{array}{llll}1.45 & 4.87 & 1.222 & 900 .\end{array}$

$\begin{array}{llll}5.55 & 18.30 & 4.660 & 900 .\end{array}$

$\begin{array}{llll}4.10 & 13.50 & 3.440 & 900 .\end{array}$

$\begin{array}{llll}6.08 & 24.54 & 6.068 & 900 .\end{array}$

$\begin{array}{rrrr}4.13 & 16.81 & 4.226 & 900 . \\ 2.24 & 9.01 & 2.240 & 900 .\end{array}$

$\begin{array}{llll}4.00 & 13.56 & 3.320 & 900 .\end{array}$

$\begin{array}{llll}3.80 & 12.70 & 3.160 & 900 \text {. }\end{array}$

$\begin{array}{llll}6.01 & 18.90 & 4.720 & 900 .\end{array}$

$\begin{array}{llll}1.91 & 6.25 & 1.604 & 900 .\end{array}$

$\begin{array}{llll}7.15 & 32.30 & 8.600 & 900 .\end{array}$

$\begin{array}{llll}7.15 & 32.30 & 8.600 & 900 .\end{array}$

$\begin{array}{rrrr}6.84 & 18.60 & 4.440 & 900 . \\ 1.79 & 5.05 & 1.258 & 900 .\end{array}$

$\begin{array}{llll}1.79 & 5.05 & 1.258 & 900 . \\ 2.67 & 7.52 & 1.874 & 900 .\end{array}$

$\begin{array}{llll}4.86 & 13.70 & 3.420 & 900 .\end{array}$

$\begin{array}{llll}2.03 & 5.88 & 1.396 & 900 .\end{array}$

$\begin{array}{llll}4.05 & 16.35 & 4.058 & 900 .\end{array}$

$\begin{array}{llll}2.63 & 12.20 & 3.110 & 900 .\end{array}$

$\begin{array}{llll}7.30 & 28.90 & 7.380 & 900 .\end{array}$

$\begin{array}{rrrr}8.69 & 29.10 & 7.310 & 900 . \\ 1.69 & 7.07 & 2.020 & 900 .\end{array}$ 


\begin{tabular}{|c|c|c|c|c|c|c|c|}
\hline 54 & 56 & .275 & .955 & 0.732 & & & 900. \\
\hline 55 & 56 & .488 & 1.51 & 0.374 & & & 900. \\
\hline 56 & 57 & 3.43 & 9.66 & 2.420 & & & 900. \\
\hline 50 & 57 & 4.74 & 13.40 & 3.320 & & & 900. \\
\hline 56 & 58 & 3.43 & 9.66 & 2.420 & & & 900. \\
\hline 51 & 58 & 2.55 & 7.19 & 1.788 & & & 900. \\
\hline 54 & 59 & 5.03 & 22.93 & 5.980 & & & 900. \\
\hline 56 & 59 & 8.25 & 25.10 & 5.690 & & & 900. \\
\hline 56 & 59 & 8.03 & 23.90 & 5.360 & & & 900. \\
\hline 55 & 59 & 4.739 & 21.58 & 5.646 & & & 900. \\
\hline 59 & 60 & 3.17 & 14.50 & 3.760 & & & 900. \\
\hline 59 & 61 & 3.28 & 15.00 & 3.880 & & & 900. \\
\hline 60 & 61 & .264 & 1.35 & 1.456 & & & 900. \\
\hline 60 & 62 & 1.23 & 5.61 & 1.468 & & & 900. \\
\hline 61 & 62 & .824 & 3.76 & 0.980 & & & 900. \\
\hline 63 & 59 & 0.00 & 3.86 & 0.000 & 1.0 & .951 .05 & 900. \\
\hline 63 & 64 & .172 & 2.00 & 21.60 & & & 900. \\
\hline 64 & 61 & 0.00 & 2.68 & 0.000 & 1.0 & .951 .05 & 900. \\
\hline 38 & 65 & .901 & 9.86 & 114.6 & & & 900. \\
\hline 64 & 65 & .269 & 3.02 & 38.00 & & & 900. \\
\hline 49 & 66 & 1.80 & 9.19 & 2.480 & & & 900. \\
\hline 49 & 66 & 1.80 & 9.19 & 2.480 & & & 900. \\
\hline 62 & 66 & 4.82 & 21.80 & 5.780 & & & 900. \\
\hline 62 & 67 & 2.58 & 11.70 & 3.100 & & & 900. \\
\hline 65 & 66 & 0.00 & 3.70 & 0.000 & 1.0 & .951 .05 & 900. \\
\hline 66 & 67 & 2.24 & 10.15 & 2.682 & & & 900. \\
\hline 65 & 68 & .138 & 1.60 & 63.80 & & & 900. \\
\hline 47 & 69 & 8.44 & 27.78 & 7.092 & & & 900. \\
\hline 49 & 69 & 9.85 & 32.40 & 8.280 & & & 900. \\
\hline 68 & 69 & 0.00 & 3.70 & 0.000 & 1.0 & .951 .05 & 900. \\
\hline 69 & 70 & 3.00 & 12.70 & 12.20 & & & 900. \\
\hline 24 & 70 & 10.221 & 141.15 & 10.198 & & & 900. \\
\hline 70 & 71 & .882 & 3.55 & 0.878 & & & 900. \\
\hline 24 & 72 & 4.88 & 19.60 & 4.880 & & & 900. \\
\hline 71 & 72 & 4.46 & 18.00 & 4.444 & & & 900. \\
\hline 71 & 73 & .866 & 4.54 & 1.178 & & & 900. \\
\hline 70 & 74 & 4.01 & 13.23 & 3.368 & & & 900. \\
\hline 70 & 75 & 4.28 & 14.10 & 3.600 & & & 900. \\
\hline 69 & 75 & 4.05 & 12.20 & 12.40 & & & 900. \\
\hline 74 & 75 & 1.23 & 4.06 & 1.034 & & & 900. \\
\hline 76 & 77 & 4.44 & 14.80 & 3.680 & & & 900. \\
\hline 69 & 77 & 3.09 & 10.10 & 10.38 & & & 900. \\
\hline 75 & 77 & 6.01 & 19.99 & 4.978 & & & 900. \\
\hline 77 & 78 & .376 & 1.24 & 1.264 & & & 900. \\
\hline 78 & 79 & .546 & 2.44 & 0.648 & & & 900. \\
\hline 77 & 80 & 1.70 & 4.85 & 4.720 & & & 900. \\
\hline 77 & 80 & 2.94 & 10.50 & 2.280 & & & 900. \\
\hline 79 & 80 & 1.56 & 7.04 & 1.870 & & & 900. \\
\hline 68 & 81 & .175 & 2.02 & 80.80 & & & 900. \\
\hline 81 & 80 & 0.00 & 3.70 & 0.000 & 1.0 & .951 .05 & 900. \\
\hline 77 & 82 & 2.98 & 8.53 & 8.174 & & & 900. \\
\hline 82 & 83 & 1.12 & 3.665 & 3.796 & & & 900. \\
\hline 83 & 84 & 6.25 & 13.20 & 2.580 & & & 900. \\
\hline 83 & 85 & 4.30 & 14.80 & 3.480 & & & 900. \\
\hline 84 & 85 & 3.02 & 6.41 & 1.234 & & & 900. \\
\hline 85 & 86 & 3.50 & 12.30 & 2.760 & & & 900. \\
\hline 86 & 87 & 2.828 & 20.74 & 4.450 & & & 900. \\
\hline 85 & 88 & 2.00 & 10.20 & 2.760 & & & 900. \\
\hline 85 & 89 & 2.39 & 17.30 & 4.700 & & & 900. \\
\hline 88 & 89 & 1.39 & 7.12 & 1.934 & & & 900. \\
\hline 89 & 90 & 5.18 & 18.80 & 5.280 & & & 900. \\
\hline 89 & 90 & 2.38 & 9.97 & 10.60 & & & 900. \\
\hline 90 & 91 & 2.54 & 8.36 & 2.140 & & & 900. \\
\hline 89 & 92 & 0.99 & 5.05 & 5.480 & & & 900. \\
\hline 89 & 92 & 3.93 & 15.81 & 4.140 & & & 900. \\
\hline 91 & 92 & 3.87 & 12.72 & 3.268 & & & 900. \\
\hline 92 & 93 & 2.58 & 8.48 & 2.180 & & & 900. \\
\hline 92 & 94 & 4.81 & 15.80 & 4.060 & & & 900. \\
\hline 93 & 94 & 2.23 & 7.32 & 1.876 & & & 900. \\
\hline 94 & 95 & 1.32 & 4.34 & 1.110 & & & 900. \\
\hline 80 & 96 & 3.56 & 18.20 & 4.940 & & & 900. \\
\hline 82 & 96 & 1.62 & 5.30 & 5.440 & & & 900. \\
\hline 94 & 96 & 2.69 & 8.69 & 2.300 & & & 900 \\
\hline 80 & 97 & 1.83 & 9.34 & 2.540 & & & \\
\hline
\end{tabular}




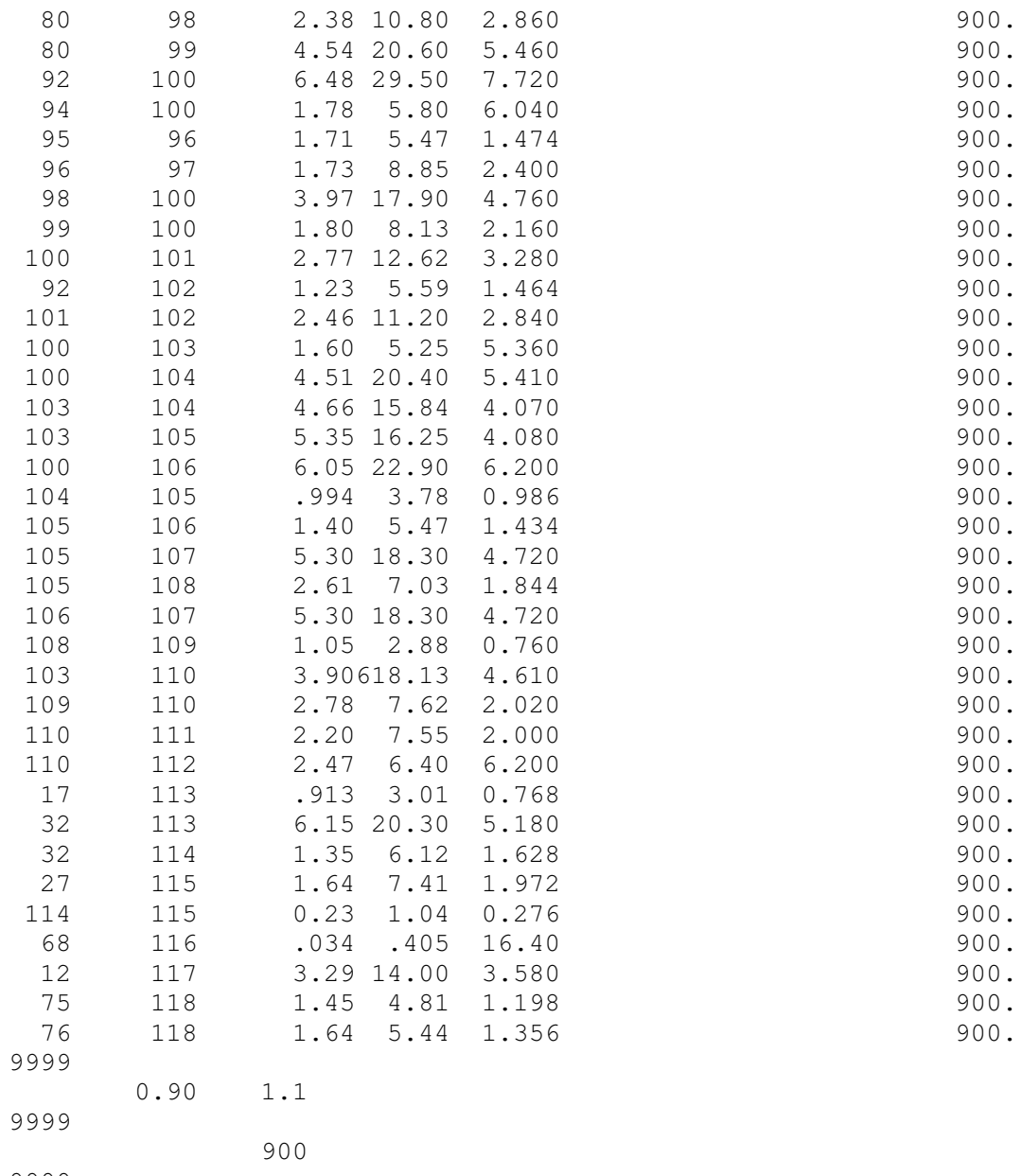

9999 


\section{ESTADO FINAL DOS SISTEMAS ELÉTRICOS}

- $\quad$ Estado final do sistema de 6 barras

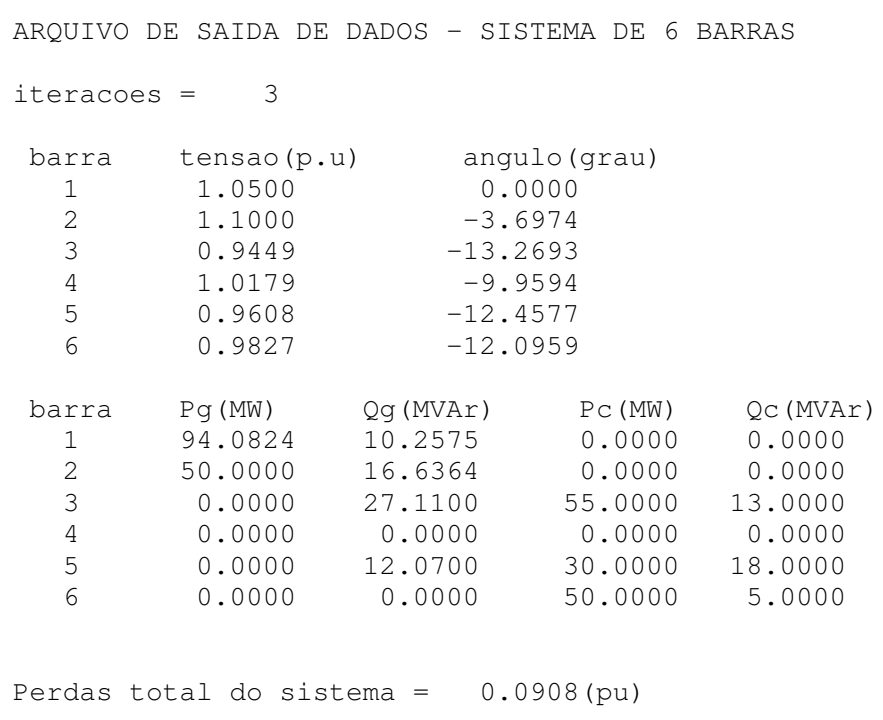


- $\quad$ Estado final do sistema de 8 barras

ARQUIVO DE SAIDA DE DADOS - SISTEMA DE 8 BARRAS

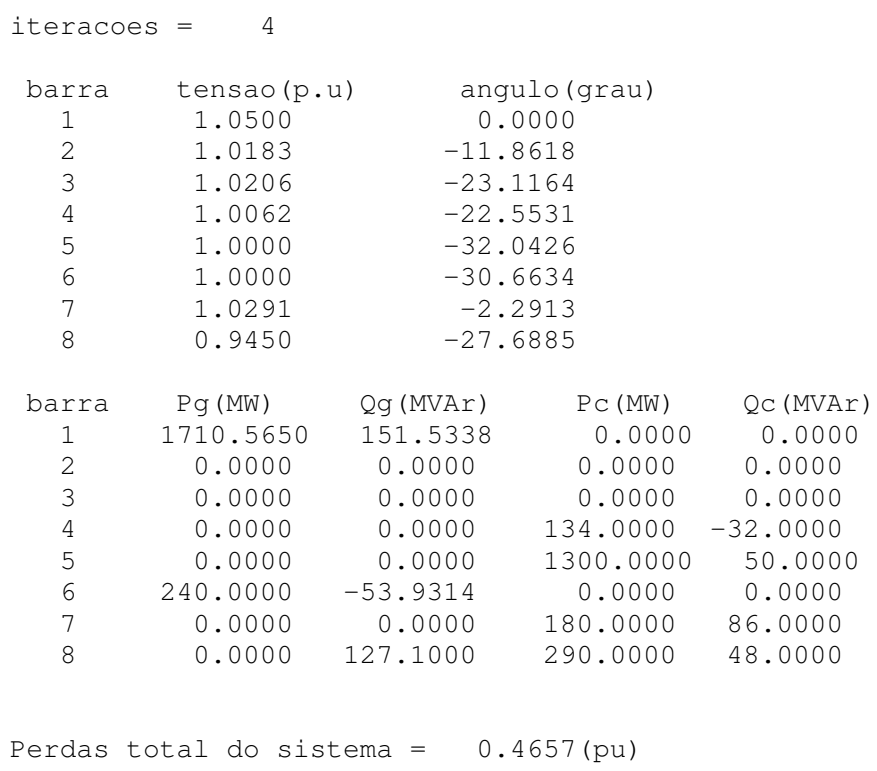




\section{- $\quad$ Estado final do sistema AEP - 30barras}

CONVERGENCIA FINAL

ITERACAO
ERRO MAX
FC P PRRA

CEPEL - Centro de Pesquisas de Energia Eletrica - ANAREDE V07-0899B

RELATORIO DE BARRAS CA * AREA 1 *

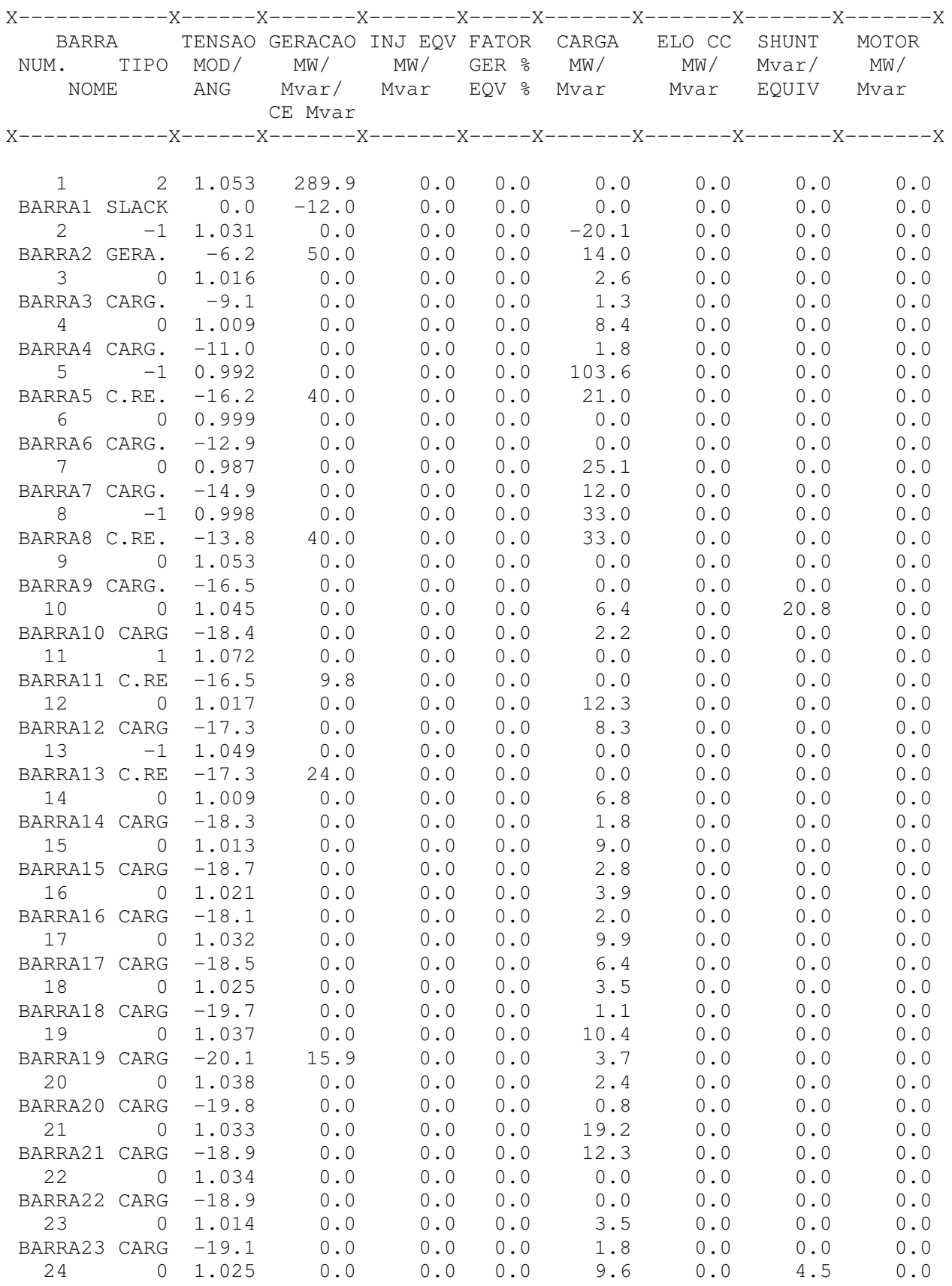




$\begin{array}{lrllllllll}\text { BARRA24 } & \text { CARG } & -19.4 & 0.0 & 0.0 & 0.0 & 7.4 & 0.0 & 0.0 & 0.0 \\ 25 & 0 & 1.052 & 0.0 & 0.0 & 0.0 & 0.0 & 0.0 & 0.0 & 0.0 \\ \text { BARRA25 } & \text { CARG } & -19.2 & 0.0 & 0.0 & 0.0 & 0.0 & 0.0 & 0.0 & 0.0 \\ 26 & 0 & 1.059 & 0.0 & 0.0 & 0.0 & 3.9 & 0.0 & 0.0 & 0.0 \\ \text { BARRA26 } & \text { CARG } & -20.6 & 7.4 & 0.0 & 0.0 & 2.5 & 0.0 & 0.0 & 0.0 \\ 27 & 0 & 1.063 & 0.0 & 0.0 & 0.0 & 0.0 & 0.0 & 0.0 & 0.0 \\ \text { BARRA27 } & \text { CARG } & -18.4 & 0.0 & 0.0 & 0.0 & 0.0 & 0.0 & 0.0 & 0.0 \\ 28 & 0 & 0.998 & 0.0 & 0.0 & 0.0 & 0.0 & 0.0 & 0.0 & 0.0 \\ \text { BARRA28 } & \text { CARG } & -13.8 & 0.0 & 0.0 & 0.0 & 0.0 & 0.0 & 0.0 & 0.0 \\ \text { 29 } & 0 & 1.057 & 0.0 & 0.0 & 0.0 & 2.6 & 0.0 & 0.0 & 0.0 \\ \text { BARRA29 } & \text { CARG } & -20.1 & 0.0 & 0.0 & 0.0 & 1.0 & 0.0 & 0.0 & 0.0 \\ \text { 30 } & 0 & 1.061 & 0.0 & 0.0 & 0.0 & 11.7 & 0.0 & 0.0 & 0.0 \\ \text { BARRA30 } & \text { CARG } & -21.4 & 9.5 & 0.0 & 0.0 & 2.1 & 0.0 & 0.0 & 0.0\end{array}$

CEPEL - Centro de Pesquisas de Energia Eletrica - ANAREDE V07-0899B

RELAtorio DE CIRC. CA DO SISt. * AREA 1 *

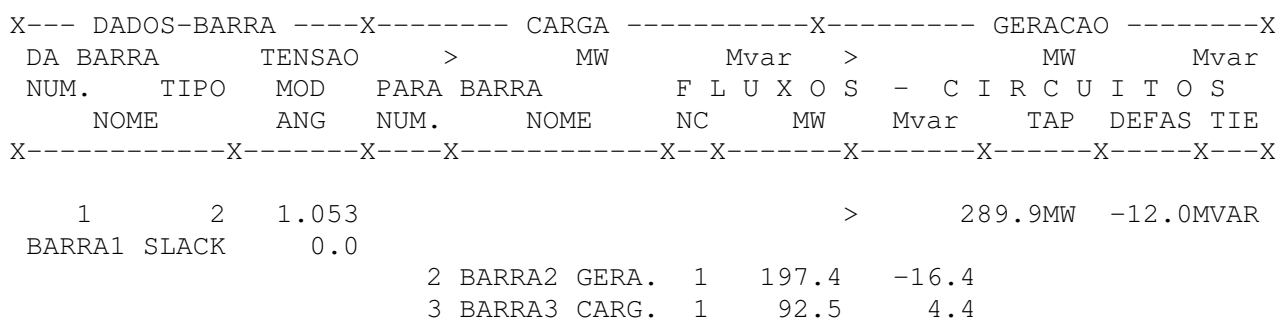

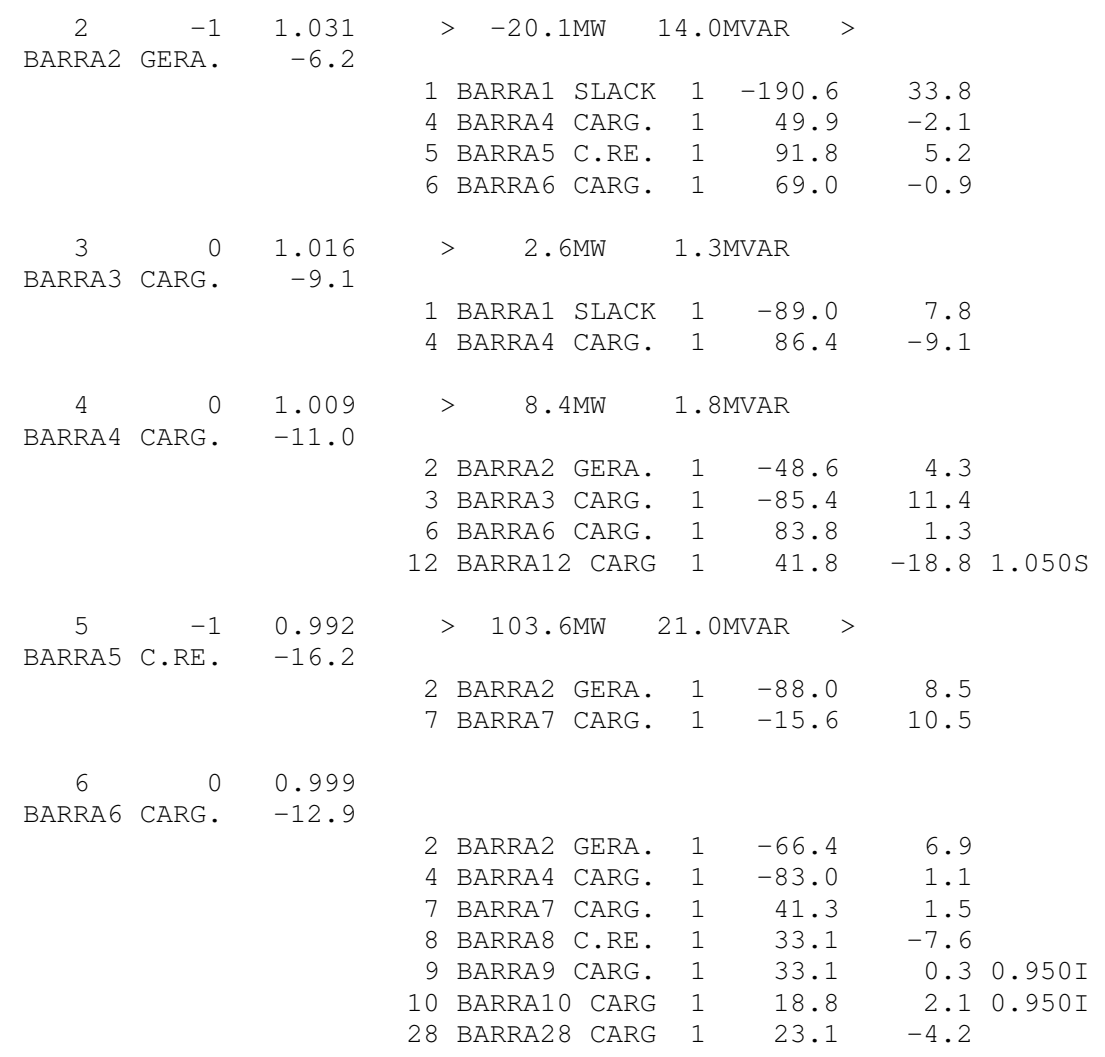

$\begin{array}{crr}7 & 0 & 0.987 \\ \text { BARRA7 } & \text { CARG. } & -14.9\end{array}$

$>\quad 25.1 \mathrm{MW} \quad$ 12.0MVAR

5 BARRA5 C.RE. $11015.8 \quad-11.1$

6 BARRA6 CARG. $1 \quad-40.9 \quad-0.9$

$\begin{array}{crr}8 & -1 & 0.998 \\ \text { BARRA8 } & \text { C.RE. } & -13.8\end{array}$

$>33.0 \mathrm{MW} 33.0 \mathrm{MVAR}>$

40. OMVAR

6 BARRA6 CARG. $1 \quad-33.0 \quad 7.7$ 
28 BARRA28 CARG $1 \quad 0.0 \quad-0.7$

$\begin{array}{lll}9 & 0 & 1.053\end{array}$

BARRA9 CARG. $\quad-16.5$

$\begin{array}{rlrrr}6 & \text { BARRA 6 CARG. } & 1 & -33.1 & 1.7 \\ 10 & \text { BARRA10 CARG } & 1 & 33.1 & 7.9 \\ 11 & \text { BARRA11 C.RE } & 1 & 0.0 & -9.6\end{array}$

$\begin{array}{lll}10 & 0 & 1.045\end{array}$

BARRA10 CARG -18.4

\section{2MVAR}

$\begin{array}{rll}6 & \text { BARRA6 } & \text { CARG. } \\ 9 & \text { BARRA9 } & \text { CARG. } \\ 17 & \text { BARRA17 } & \text { CARG } \\ 20 & \text { BARRA20 } & \text { CARG } \\ 21 & \text { BARRA21 } & \text { CARG } \\ 22 & \text { BARRA22 } & \text { CARG }\end{array}$

$\begin{array}{rr}-18.8 & -0.3 \\ -33.1 & -6.7 \\ 8.5 & 13.2 \\ 11.8 & -1.5 \\ 17.1 & 9.7 \\ 8.2 & 4.2\end{array}$

$\begin{array}{lll}11 & 1 & 1.072\end{array}$

BARRA11 C.RE -16.5

9 BARRA9 CARG. $1 \quad 0.0 \quad 9.8$

$\begin{array}{lll}12 & 0 & 1.017\end{array}$

BARRA12 CARG -17.3

\section{$>\quad 12.3 \mathrm{MW} \quad 8.3 \mathrm{MVAR}$}

4 BARRA4 CARG. $1 \quad-41.8 \quad 24.6$

13 BARRA13 C.RE $1 \quad 0.0 \quad-23.3$

14 BARRA14 CARG $1 \quad 7.3 \quad-0.3$

15 BARRA15 CARG 1 16.8 -4.9

16 BARRA16 CARG $1 \quad 5.5 \quad-4.4$

$\begin{array}{lll}13 & -1 & 1.049\end{array}$

BARRA13 C.RE -17.3

12 BARRA12 CARG $1 \quad 0.0 \quad 24.0$

1401.009

$\begin{array}{ll}>\quad 6.8 \mathrm{MW} & 1.8 \mathrm{MVAR}\end{array}$

BARRA14 CARG -18.3

12 BARRA12 CARG $1 \quad-7.2 \quad 0.5$

15 BARRA15 CARG 1 0.4

$\begin{array}{lrr}15 & 0 & 1.013\end{array}$

BARRA15 CARG -18.7

$\begin{array}{lllrrr}12 & \text { BARRA12 } & \text { CARG } & 1 & -16.6 & 5.3 \\ 14 & \text { BARRA14 } & \text { CARG } & 1 & -0.4 & 2.3 \\ 18 & \text { BARRA18 } & \text { CARG } & 1 & 4.8 & -8.2 \\ 23 & \text { BARRA23 } & \text { CARG } & 1 & 3.2 & -2.2\end{array}$

$\begin{array}{lll}16 & 0 & 1.021\end{array}$

BARRA16 CARG -18.1

\section{3. $9 \mathrm{MW} \quad 2.0 \mathrm{MVAR}$}

12 BARRA12 CARG $1 \quad-5.4 \quad 4.5$

17 BARRA17 CARG 1 1 $1.5 \quad-6.5$

$\begin{array}{lrr}17 & 0 & 1.032\end{array}$

9.9MW 6.4MVAR

BARRA17 CARG -18.5

10 BARRA10 CARG $1 \quad-8.4 \quad-13.0$

16 BARRA16 CARG $1 \quad-1.5 \quad 6.6$

$18 \quad 0 \quad 1.025$

BARRA18 CARG -19.7

$>\quad 3.5 \mathrm{MW} \quad$ 1.1MVAR

15 BARRA15 CARG $1 \quad-4.8 \quad 8.4$

19 BARRA19 CARG 1 1 $1.3 \quad-9.5$

$\begin{array}{lrr}19 & 0 & 1.037 \\ \text { BARRA19 } & \text { CARG } & -20.1\end{array}$

$>\quad 10.4 \mathrm{MW} \quad 3.7 \mathrm{MVAR}>$

15. 9MVAR

18 BARRA18 CARG $1 \quad-1.2 \quad 9.6$

20 BARRA20 CARG $1 \quad-9.2 \quad 2.6$

$20 \quad 0 \quad 1.038$

BARRA20 CARG -19.8

$$
\text { 2. } 4 \mathrm{MW} \quad 0.8 \mathrm{MVAR}
$$

10 BARRA10 CARG $1 \quad-11.6 \quad 1.7$

19 BARRA19 CARG $1 \quad 9.2 \quad-2.5$

$21 \quad 0 \quad 1.033$

BARRA21 CARG -18.9

10 BARRA10 CARG $1 \quad-16.9 \quad-9.4$

9. 8MVAR

24. OMVAR

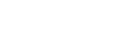


22 BARRA22 CARG $1 \quad-2.3 \quad-2.9$

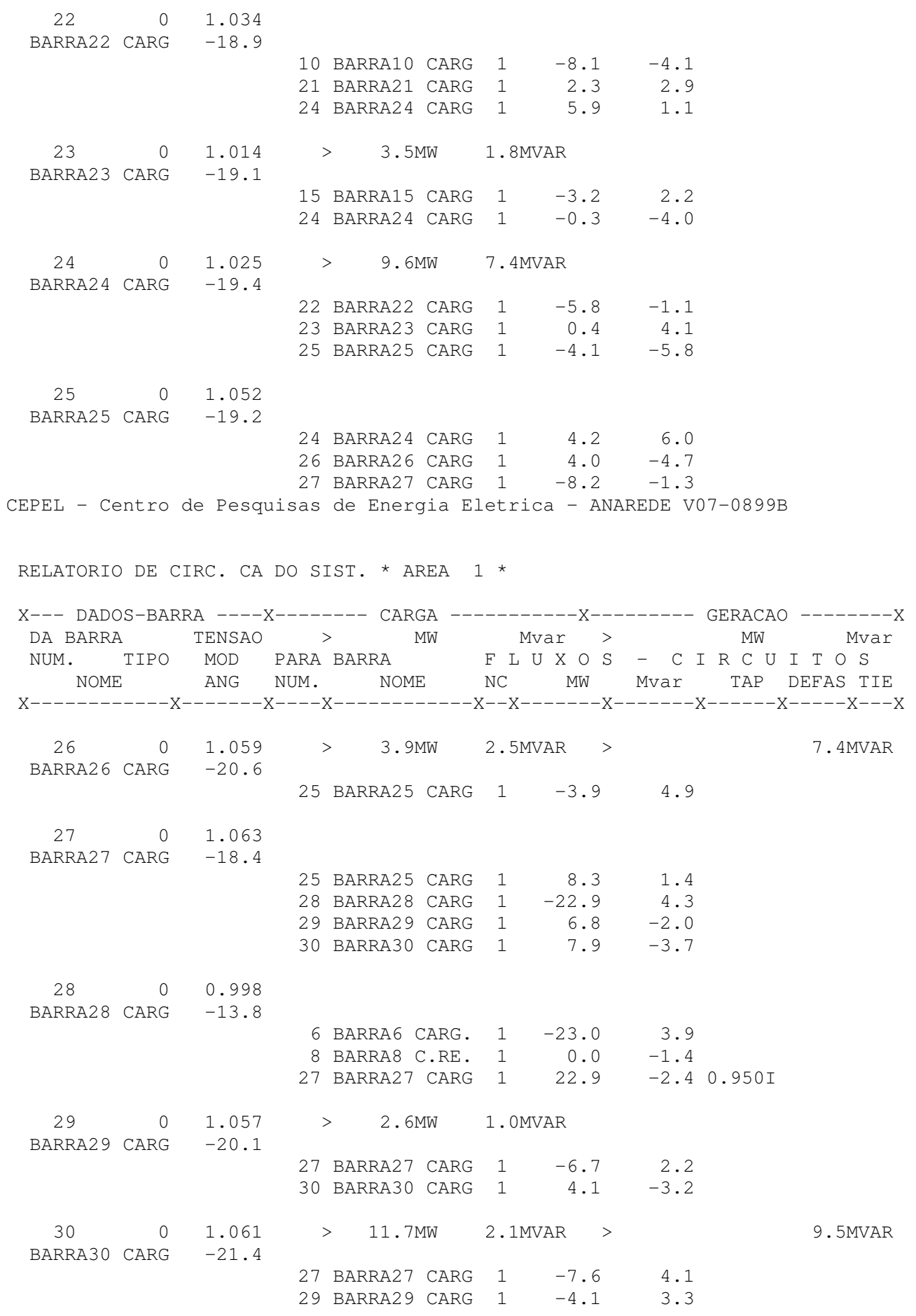

TOTAIS DA AREA 1

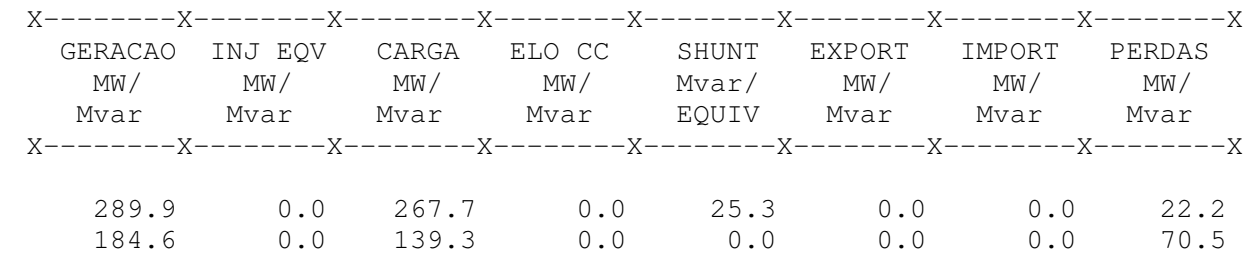


- $\quad$ Estado final do sistema IEEE118

CONVERGENCIA FINAL

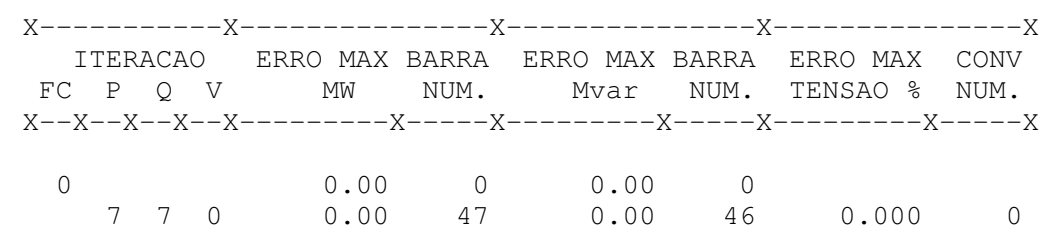

CEPEL - Centro de Pesquisas de Energia Eletrica - ANAREDE V07-0899B

RELATORIO DE BARRAS CA * AREA 1 *

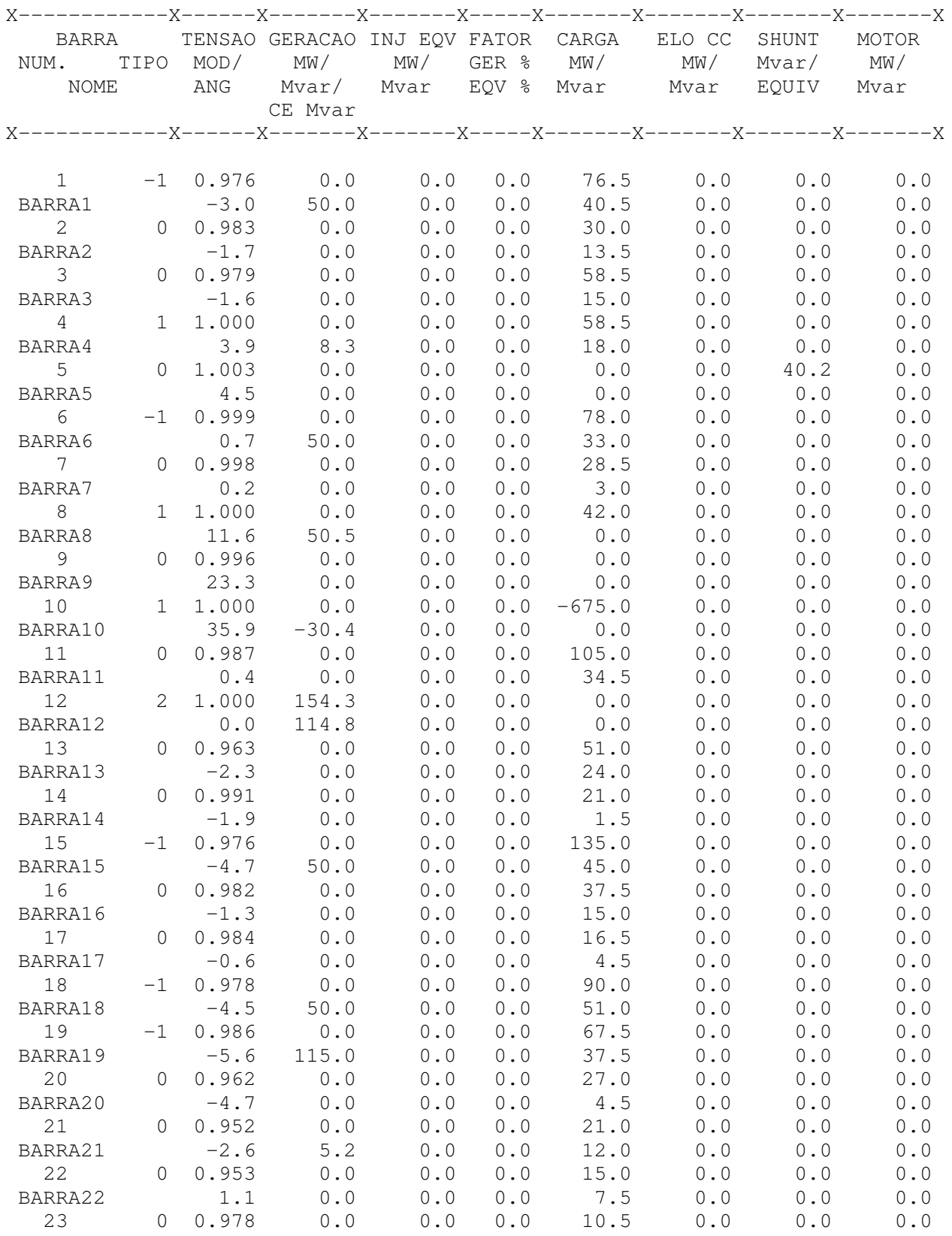




\begin{tabular}{|c|c|c|c|c|c|c|c|c|c|}
\hline BARRA23 & & 8.3 & 0.0 & 0.0 & 0.0 & 4.5 & 0.0 & 0.0 & 0.0 \\
\hline 24 & 0 & 0.984 & 0.0 & 0.0 & 0.0 & 19.5 & 0.0 & 0.0 & 0.0 \\
\hline BARRA2 4 & & 7.2 & 0.0 & 0.0 & 0.0 & 0.0 & 0.0 & 0.0 & 0.0 \\
\hline 25 & 1 & 1.000 & 0.0 & 0.0 & 0.0 & -330.0 & 0.0 & 0.0 & 0.0 \\
\hline BARRA2 5 & & 20.5 & 11.9 & 0.0 & 0.0 & 0.0 & 0.0 & 0.0 & 0.0 \\
\hline 26 & 1 & 1.000 & 0.0 & 0.0 & 0.0 & -471.0 & 0.0 & 0.0 & 0.0 \\
\hline BARRA2 6 & & 23.7 & -23.5 & 0.0 & 0.0 & 0.0 & 0.0 & 0.0 & 0.0 \\
\hline 27 & -1 & 0.967 & 0.0 & 0.0 & 0.0 & 107.0 & 0.0 & 0.0 & 0.0 \\
\hline BARRA27 & & -0.2 & 50.0 & 0.0 & 0.0 & 19.5 & 0.0 & 0.0 & 0.0 \\
\hline 28 & 0 & 0.957 & 0.0 & 0.0 & 0.0 & 25.5 & 0.0 & 0.0 & 0.0 \\
\hline BARRA2 8 & & -2.5 & 0.0 & 0.0 & 0.0 & 10.5 & 0.0 & 0.0 & 0.0 \\
\hline 29 & 0 & 0.959 & 0.0 & 0.0 & 0.0 & 36.0 & 0.0 & 0.0 & 0.0 \\
\hline BARRA29 & & -3.8 & 0.0 & 0.0 & 0.0 & 6.0 & 0.0 & 0.0 & 0.0 \\
\hline 30 & 0 & 0.998 & 0.0 & 0.0 & 0.0 & 0.0 & 0.0 & 0.0 & 0.0 \\
\hline BARRA30 & & 7.3 & 0.0 & 0.0 & 0.0 & 0.0 & 0.0 & 0.0 & 0.0 \\
\hline 31 & -1 & 0.965 & 0.0 & 0.0 & 0.0 & 54.0 & 0.0 & 0.0 & 0.0 \\
\hline BARRA31 & & -3.5 & 50.0 & 0.0 & 0.0 & 40.5 & 0.0 & 0.0 & 0.0 \\
\hline 32 & -1 & 0.977 & 0.0 & 0.0 & 0.0 & 88.5 & 0.0 & 0.0 & 0.0 \\
\hline BARRA32 & & -1.1 & 100.0 & 0.0 & 0.0 & 34.5 & 0.0 & 0.0 & 0.0 \\
\hline 33 & 0 & 0.964 & 0.0 & 0.0 & 0.0 & 34.5 & 0.0 & 0.0 & 0.0 \\
\hline BARRA33 & & -6.1 & 0.0 & 0.0 & 0.0 & 13.5 & 0.0 & 0.0 & 0.0 \\
\hline 34 & 0 & 0.976 & 0.0 & 0.0 & 0.0 & 88.5 & 0.0 & 13.3 & 0.0 \\
\hline BARRA 34 & & -5.8 & 0.0 & 0.0 & 0.0 & 39.0 & 0.0 & 0.0 & 0.0 \\
\hline 35 & 0 & 0.975 & 0.0 & 0.0 & 0.0 & 49.5 & 0.0 & 0.0 & 0.0 \\
\hline BARRA35 & & -6.6 & 0.0 & 0.0 & 0.0 & 13.5 & 0.0 & 0.0 & 0.0 \\
\hline 36 & -1 & 0.976 & 0.0 & 0.0 & 0.0 & 46.5 & 0.0 & 0.0 & 0.0 \\
\hline BARRA 36 & & -6.6 & 50.0 & 0.0 & 0.0 & 25.5 & 0.0 & 0.0 & 0.0 \\
\hline 37 & 0 & 0.982 & 0.0 & 0.0 & 0.0 & 0.0 & 0.0 & 24.1 & 0.0 \\
\hline BARRA37 & & -5.0 & 0.0 & 0.0 & 0.0 & 0.0 & 0.0 & 0.0 & 0.0 \\
\hline 38 & 0 & 0.997 & 0.0 & 0.0 & 0.0 & 0.0 & 0.0 & 0.0 & 0.0 \\
\hline BARRA38 & & 3.5 & 0.0 & 0.0 & 0.0 & 0.0 & 0.0 & 0.0 & 0.0 \\
\hline 39 & 0 & 0.975 & 0.0 & 0.0 & 0.0 & 40.5 & 0.0 & 0.0 & 0.0 \\
\hline BARRA39 & & -11.3 & 0.0 & 0.0 & 0.0 & 16.5 & 0.0 & 0.0 & 0.0 \\
\hline 40 & -1 & 0.992 & 0.0 & 0.0 & 0.0 & 99.0 & 0.0 & 0.0 & 0.0 \\
\hline BARRA 40 & & -13.5 & 150.0 & 0.0 & 0.0 & 34.5 & 0.0 & 0.0 & 0.0 \\
\hline 41 & 0 & 0.975 & 0.0 & 0.0 & 0.0 & 55.5 & 0.0 & 0.0 & 0.0 \\
\hline BARRA 41 & & -14.2 & 0.0 & 0.0 & 0.0 & 15.0 & 0.0 & 0.0 & 0.0 \\
\hline 42 & -1 & 0.970 & 0.0 & 0.0 & 0.0 & 144.0 & 0.0 & 0.0 & 0.0 \\
\hline BARRA 42 & & -11.9 & 50.0 & 0.0 & 0.0 & 34.5 & 0.0 & 0.0 & 0.0 \\
\hline 43 & 0 & 0.950 & 0.0 & 0.0 & 0.0 & 27.0 & 0.0 & 0.0 & 0.0 \\
\hline BARRA 43 & & -6.6 & 0.0 & 0.0 & 0.0 & 10.5 & 0.0 & 0.0 & 0.0 \\
\hline 44 & 0 & 0.946 & 0.0 & 0.0 & 0.0 & 24.0 & 0.0 & 9.0 & 0.0 \\
\hline BARRA 44 & & -3.9 & 0.0 & 0.0 & 0.0 & 12.0 & 0.0 & 0.0 & 0.0 \\
\hline 45 & 0 & 0.951 & 0.0 & 0.0 & 0.0 & 79.5 & 0.0 & 9.0 & 0.0 \\
\hline BARRA 45 & & -1.5 & 3.6 & 0.0 & 0.0 & 33.0 & 0.0 & 0.0 & 0.0 \\
\hline 46 & -1 & 0.999 & 0.0 & 0.0 & 0.0 & 13.5 & 0.0 & 10.0 & 0.0 \\
\hline BARRA 46 & & 2.2 & 50.0 & 0.0 & 0.0 & 15.0 & 0.0 & 0.0 & 0.0 \\
\hline 47 & 0 & 0.992 & 0.0 & 0.0 & 0.0 & 51.0 & 0.0 & 0.0 & 0.0 \\
\hline BARRA 47 & & 5.7 & 0.0 & 0.0 & 0.0 & 0.0 & 0.0 & 0.0 & 0.0 \\
\hline 48 & 0 & 0.995 & 0.0 & 0.0 & 0.0 & 30.0 & 0.0 & 14.9 & 0.0 \\
\hline BARRA 48 & & 5.0 & 0.0 & 0.0 & 0.0 & 16.5 & 0.0 & 0.0 & 0.0 \\
\hline 49 & 1 & 1.000 & 0.0 & 0.0 & 0.0 & -176.0 & 0.0 & 0.0 & 0.0 \\
\hline BARRA 49 & & 6.6 & 135.4 & 0.0 & 0.0 & 45.0 & 0.0 & 0.0 & 0.0 \\
\hline 50 & 0 & 0.987 & 0.0 & 0.0 & 0.0 & 25.5 & 0.0 & 0.0 & 0.0 \\
\hline BARRA 50 & & 3.1 & 0.0 & 0.0 & 0.0 & 6.0 & 0.0 & 0.0 & 0.0 \\
\hline 51 & 0 & 0.966 & 0.0 & 0.0 & 0.0 & 25.5 & 0.0 & 0.0 & 0.0 \\
\hline BARRA 51 & & -1.3 & 0.0 & 0.0 & 0.0 & 12.0 & 0.0 & 0.0 & 0.0 \\
\hline 52 & 0 & 0.959 & 0.0 & 0.0 & 0.0 & 27.0 & 0.0 & 0.0 & 0.0 \\
\hline BARRA 52 & & -2.8 & 0.0 & 0.0 & 0.0 & 7.5 & 0.0 & 0.0 & 0.0 \\
\hline 53 & 0 & 0.967 & 0.0 & 0.0 & 0.0 & 34.5 & 0.0 & 0.0 & 0.0 \\
\hline BARRA 53 & & -4.5 & 0.0 & 0.0 & 0.0 & 16.5 & 0.0 & 0.0 & 0.0 \\
\hline 54 & 1 & 1.000 & 0.0 & 0.0 & 0.0 & 97.5 & 0.0 & 0.0 & 0.0 \\
\hline BARRA 54 & & -3.4 & 122.3 & 0.0 & 0.0 & 48.0 & 0.0 & 0.0 & 0.0 \\
\hline 55 & 1 & 1.000 & 0.0 & 0.0 & 0.0 & 94.5 & 0.0 & 0.0 & 0.0 \\
\hline BARRA 55 & & -3.8 & 79.4 & 0.0 & 0.0 & 33.0 & 0.0 & 0.0 & 0.0 \\
\hline 56 & 1 & 1.000 & 0.0 & 0.0 & 0.0 & 126.0 & 0.0 & 0.0 & 0.0 \\
\hline BARRA 56 & & -3.6 & 143.4 & 0.0 & 0.0 & 27.0 & 0.0 & 0.0 & 0.0 \\
\hline 57 & 0 & 0.989 & 0.0 & 0.0 & 0.0 & 18.0 & 0.0 & 0.0 & 0.0 \\
\hline BARRA 57 & & -1.4 & 0.0 & 0.0 & 0.0 & 4.5 & 0.0 & 0.0 & 0.0 \\
\hline 58 & 0 & 0.977 & 0.0 & 0.0 & 0.0 & 18.0 & 0.0 & 0.0 & 0.0 \\
\hline BARRA 58 & & -2.7 & 0.0 & 0.0 & 0.0 & 4.5 & 0.0 & 0.0 & 0.0 \\
\hline 59 & -1 & 0.953 & 0.0 & 0.0 & 0.0 & 183.0 & 0.0 & 0.0 & 0.0 \\
\hline BARRA 59 & & 4.7 & 50.0 & 0.0 & 0.0 & 169.5 & 0.0 & 0.0 & 0.0 \\
\hline 60 & 0 & 0.993 & 0.0 & 0.0 & 0.0 & 117.0 & 0.0 & 0.0 & 0.0 \\
\hline
\end{tabular}




\begin{tabular}{|c|c|c|c|c|c|c|c|c|c|}
\hline BARRA 60 & & 10.7 & 0.0 & 0.0 & 0.0 & 4.5 & 0.0 & 0.0 & 0.0 \\
\hline 61 & 1 & 1.000 & 0.0 & 0.0 & 0.0 & -240.0 & 0.0 & 0.0 & 0.0 \\
\hline BARRA 61 & & 12.0 & 38.2 & 0.0 & 0.0 & 0.0 & 0.0 & 0.0 & 0.0 \\
\hline 62 & -1 & 0.997 & 0.0 & 0.0 & 0.0 & 116.0 & 0.0 & 0.0 & 0.0 \\
\hline BARRA 62 & & 11.1 & 50.0 & 0.0 & 0.0 & 21.0 & 0.0 & 0.0 & 0.0 \\
\hline 63 & 0 & 0.995 & 0.0 & 0.0 & 0.0 & 0.0 & 0.0 & 0.0 & 0.0 \\
\hline BARRA 63 & & 10.4 & 0.0 & 0.0 & 0.0 & 0.0 & 0.0 & 0.0 & 0.0 \\
\hline 64 & 0 & 0.998 & 0.0 & 0.0 & 0.0 & 0.0 & 0.0 & 0.0 & 0.0 \\
\hline BARRA 64 & & 13.1 & 0.0 & 0.0 & 0.0 & 0.0 & 0.0 & 0.0 & 0.0 \\
\hline 65 & 1 & 1.000 & 0.0 & 0.0 & 0.0 & -587.0 & 0.0 & 0.0 & 0.0 \\
\hline BARRA 65 & & 18.3 & -120.9 & 0.0 & 0.0 & 0.0 & 0.0 & 0.0 & 0.0 \\
\hline 66 & 1 & 1.000 & 0.0 & 0.0 & 0.0 & -530.0 & 0.0 & 0.0 & 0.0 \\
\hline BARRA 66 & & 18.0 & -27.5 & 0.0 & 0.0 & 27.0 & 0.0 & 0.0 & 0.0 \\
\hline 67 & 0 & 0.987 & 0.0 & 0.0 & 0.0 & 42.0 & 0.0 & 0.0 & 0.0 \\
\hline BARRA 67 & & 13.5 & 0.0 & 0.0 & 0.0 & 10.5 & 0.0 & 0.0 & 0.0 \\
\hline 68 & 0 & 1.003 & 0.0 & 0.0 & 0.0 & 0.0 & 0.0 & 0.0 & 0.0 \\
\hline BARRA 68 & & 18.3 & 0.0 & 0.0 & 0.0 & 0.0 & 0.0 & 0.0 & 0.0 \\
\hline 69 & 1 & 1.000 & 0.0 & 0.0 & 0.0 & -494.0 & 0.0 & 0.0 & 0.0 \\
\hline BARRA 69 & & 18.0 & -55.7 & 0.0 & 0.0 & 0.0 & 0.0 & 0.0 & 0.0 \\
\hline 70 & -1 & 0.987 & 0.0 & 0.0 & 0.0 & 99.0 & 0.0 & 0.0 & 0.0 \\
\hline BARRA 70 & & 6.6 & 50.0 & 0.0 & 0.0 & 30.0 & 0.0 & 0.0 & 0.0 \\
\hline 71 & 0 & 0.994 & 0.0 & 0.0 & 0.0 & 0.0 & 0.0 & 0.0 & 0.0 \\
\hline BARRA 71 & & 6.2 & 0.0 & 0.0 & 0.0 & 0.0 & 0.0 & 0.0 & 0.0 \\
\hline 72 & 1 & 1.000 & 0.0 & 0.0 & 0.0 & 18.0 & 0.0 & 0.0 & 0.0 \\
\hline BARRA 72 & & 5.4 & 11.5 & 0.0 & 0.0 & 0.0 & 0.0 & 0.0 & 0.0 \\
\hline 73 & 1 & 1.000 & 0.0 & 0.0 & 0.0 & 9.0 & 0.0 & 0.0 & 0.0 \\
\hline BARRA 73 & & 5.9 & 14.4 & 0.0 & 0.0 & 0.0 & 0.0 & 0.0 & 0.0 \\
\hline 74 & -1 & 0.975 & 0.0 & 0.0 & 0.0 & 102.0 & 0.0 & 11.4 & 0.0 \\
\hline BARRA 74 & & 4.4 & 80.0 & 0.0 & 0.0 & 40.5 & 0.0 & 0.0 & 0.0 \\
\hline 75 & 0 & 0.963 & 0.0 & 0.0 & 0.0 & 70.5 & 0.0 & 0.0 & 0.0 \\
\hline BARRA 75 & & 6.6 & 0.0 & 0.0 & 0.0 & 16.5 & 0.0 & 0.0 & 0.0 \\
\hline 76 & -1 & 0.960 & 0.0 & 0.0 & 0.0 & 102.0 & 0.0 & 0.0 & 0.0 \\
\hline BARRA 76 & & 4.5 & 100.0 & 0.0 & 0.0 & 54.0 & 0.0 & 0.0 & 0.0 \\
\hline 77 & -1 & 0.968 & 0.0 & 0.0 & 0.0 & 91.5 & 0.0 & 0.0 & 0.0 \\
\hline BARRA 77 & & 14.0 & 50.0 & 0.0 & 0.0 & 42.0 & 0.0 & 0.0 & 0.0 \\
\hline 78 & 0 & 0.961 & 0.0 & 0.0 & 0.0 & 107.0 & 0.0 & 0.0 & 0.0 \\
\hline BARRA 78 & & 13.6 & 0.0 & 0.0 & 0.0 & 39.0 & 0.0 & 0.0 & 0.0 \\
\hline 79 & 0 & 0.962 & 0.0 & 0.0 & 0.0 & 58.5 & 0.0 & 18.5 & 0.0 \\
\hline BARRA 79 & & 14.4 & 0.0 & 0.0 & 0.0 & 48.0 & 0.0 & 0.0 & 0.0 \\
\hline 80 & 1 & 1.000 & 0.0 & 0.0 & 0.0 & -521.0 & 0.0 & 0.0 & 0.0 \\
\hline BARRA 80 & & 18.6 & 70.4 & 0.0 & 0.0 & 39.0 & 0.0 & 0.0 & 0.0 \\
\hline 81 & 0 & 1.000 & 0.0 & 0.0 & 0.0 & 0.0 & 0.0 & 0.0 & 0.0 \\
\hline BARRA 81 & & 18.4 & 0.0 & 0.0 & 0.0 & 0.0 & 0.0 & 0.0 & 0.0 \\
\hline 82 & 0 & 0.952 & 0.0 & 0.0 & 0.0 & 81.0 & 0.0 & 18.1 & 0.0 \\
\hline BARRA 82 & & 14.6 & 0.0 & 0.0 & 0.0 & 40.5 & 0.0 & 0.0 & 0.0 \\
\hline 83 & 0 & 0.956 & 0.0 & 0.0 & 0.0 & 30.0 & 0.0 & 9.1 & 0.0 \\
\hline BARRA 83 & & 16.2 & 0.0 & 0.0 & 0.0 & 15.0 & 0.0 & 0.0 & 0.0 \\
\hline 84 & 0 & 0.972 & 0.0 & 0.0 & 0.0 & 16.5 & 0.0 & 0.0 & 0.0 \\
\hline BARRA 84 & & 19.6 & 0.0 & 0.0 & 0.0 & 10.5 & 0.0 & 0.0 & 0.0 \\
\hline 85 & -1 & 0.991 & 0.0 & 0.0 & 0.0 & 36.0 & 0.0 & 0.0 & 0.0 \\
\hline BARRA 85 & & 21.6 & 80.0 & 0.0 & 0.0 & 22.5 & 0.0 & 0.0 & 0.0 \\
\hline 86 & 0 & 0.979 & 0.0 & 0.0 & 0.0 & 31.5 & 0.0 & 0.0 & 0.0 \\
\hline BARRA 86 & & 19.8 & 0.0 & 0.0 & 0.0 & 15.0 & 0.0 & 0.0 & 0.0 \\
\hline 87 & 1 & 1.000 & 0.0 & 0.0 & 0.0 & -6.0 & 0.0 & 0.0 & 0.0 \\
\hline BARRA 87 & & 20.4 & 7.3 & 0.0 & 0.0 & 0.0 & 0.0 & 0.0 & 0.0 \\
\hline 88 & 0 & 0.980 & 0.0 & 0.0 & 0.0 & 72.0 & 0.0 & 0.0 & 0.0 \\
\hline BARRA 88 & & 26.4 & 0.0 & 0.0 & 0.0 & 15.0 & 0.0 & 0.0 & 0.0 \\
\hline 89 & 1 & 1.000 & 0.0 & 0.0 & 0.0 & -911.0 & 0.0 & 0.0 & 0.0 \\
\hline BARRA 89 & & 32.6 & -70.8 & 0.0 & 0.0 & 0.0 & 0.0 & 0.0 & 0.0 \\
\hline 90 & -1 & 0.986 & 0.0 & 0.0 & 0.0 & 244.5 & 0.0 & 0.0 & 0.0 \\
\hline BARRA 90 & & 22.7 & 100.0 & 0.0 & 0.0 & 63.0 & 0.0 & 0.0 & 0.0 \\
\hline 91 & 1 & 1.000 & 0.0 & 0.0 & 0.0 & 15.0 & 0.0 & 0.0 & 0.0 \\
\hline BARRA 91 & & 22.4 & 21.7 & 0.0 & 0.0 & 0.0 & 0.0 & 0.0 & 0.0 \\
\hline 92 & -1 & 0.997 & 0.0 & 0.0 & 0.0 & 97.5 & 0.0 & 0.0 & 0.0 \\
\hline BARRA 92 & & 23.6 & 100.0 & 0.0 & 0.0 & 15.0 & 0.0 & 0.0 & 0.0 \\
\hline 93 & 0 & 0.973 & 0.0 & 0.0 & 0.0 & 18.0 & 0.0 & 0.0 & 0.0 \\
\hline BARRA 93 & & 19.5 & 0.0 & 0.0 & 0.0 & 10.5 & 0.0 & 0.0 & 0.0 \\
\hline 94 & 0 & 0.966 & 0.0 & 0.0 & 0.0 & 45.0 & 0.0 & 0.0 & 0.0 \\
\hline BARRA 94 & & 16.5 & 0.0 & 0.0 & 0.0 & 24.0 & 0.0 & 0.0 & 0.0 \\
\hline 95 & 0 & 0.950 & 0.0 & 0.0 & 0.0 & 63.0 & 0.0 & 0.0 & 0.0 \\
\hline BARRA 95 & & 15.1 & 19.0 & 0.0 & 0.0 & 46.5 & 0.0 & 0.0 & 0.0 \\
\hline 96 & 0 & 0.956 & 0.0 & 0.0 & 0.0 & 57.0 & 0.0 & 0.0 & 0.0 \\
\hline BARRA 96 & & 15.2 & 0.0 & 0.0 & 0.0 & 22.5 & 0.0 & 0.0 & 0.0 \\
\hline 97 & 0 & 0.970 & 0.0 & 0.0 & 0.0 & 22.5 & 0.0 & 0.0 & 0 . \\
\hline
\end{tabular}




\begin{tabular}{|c|c|c|c|c|c|c|c|c|c|}
\hline BARRA 97 & & 16.3 & 0.0 & 0.0 & 0.0 & 13.5 & 0.0 & 0.0 & 0.0 \\
\hline 98 & 0 & 0.986 & 0.0 & 0.0 & 0.0 & 51.0 & 0.0 & 0.0 & 0.0 \\
\hline BARRA 98 & & 15.5 & 0.0 & 0.0 & 0.0 & 12.0 & 0.0 & 0.0 & 0.0 \\
\hline 99 & 1 & 1.000 & 0.0 & 0.0 & 0.0 & 63.0 & 0.0 & 0.0 & 0.0 \\
\hline BARRA99 & & 14.2 & 11.9 & 0.0 & 0.0 & 0.0 & 0.0 & 0.0 & 0.0 \\
\hline 100 & 1 & 1.000 & 0.0 & 0.0 & 0.0 & -323.0 & 0.0 & 0.0 & 0.0 \\
\hline BARRA100 & & 15.6 & 67.6 & 0.0 & 0.0 & 27.0 & 0.0 & 0.0 & 0.0 \\
\hline 101 & 0 & 0.975 & 0.0 & 0.0 & 0.0 & 33.0 & 0.0 & 0.0 & 0.0 \\
\hline BARRA101 & & 17.7 & 0.0 & 0.0 & 0.0 & 22.5 & 0.0 & 0.0 & 0.0 \\
\hline 102 & 0 & 0.987 & 0.0 & 0.0 & 0.0 & 7.5 & 0.0 & 0.0 & 0.0 \\
\hline BARRA102 & & 21.5 & 0.0 & 0.0 & 0.0 & 4.5 & 0.0 & 0.0 & 0.0 \\
\hline 103 & 1 & 1.000 & 0.0 & 0.0 & 0.0 & -25.5 & 0.0 & 0.0 & 0.0 \\
\hline BARRA103 & & 9.5 & 100.4 & 0.0 & 0.0 & 24.0 & 0.0 & 0.0 & 0.0 \\
\hline 104 & -1 & 0.964 & 0.0 & 0.0 & 0.0 & 57.0 & 0.0 & 0.0 & 0.0 \\
\hline BARRA104 & & 5.1 & 0.0 & 0.0 & 0.0 & 37.5 & 0.0 & 0.0 & 0.0 \\
\hline 105 & -1 & 0.971 & 0.0 & 0.0 & 0.0 & 46.5 & 0.0 & 18.9 & 0.0 \\
\hline BARRA105 & & 3.2 & 20.0 & 0.0 & 0.0 & 39.0 & 0.0 & 0.0 & 0.0 \\
\hline 106 & 0 & 0.970 & 0.0 & 0.0 & 0.0 & 64.5 & 0.0 & 0.0 & 0.0 \\
\hline BARRA106 & & 2.7 & 17.2 & 0.0 & 0.0 & 24.0 & 0.0 & 0.0 & 0.0 \\
\hline 107 & -1 & 0.996 & 0.0 & 0.0 & 0.0 & 75.0 & 0.0 & 5.9 & 0.0 \\
\hline BARRA107 & & -1.9 & 60.0 & 0.0 & 0.0 & 18.0 & 0.0 & 0.0 & 0.0 \\
\hline 108 & 0 & 0.975 & 0.0 & 0.0 & 0.0 & 3.0 & 0.0 & 0.0 & 0.0 \\
\hline BARRA108 & & 1.3 & 0.0 & 0.0 & 0.0 & 1.5 & 0.0 & 0.0 & 0.0 \\
\hline 109 & 0 & 0.977 & 0.0 & 0.0 & 0.0 & 12.0 & 0.0 & 0.0 & 0.0 \\
\hline BARRA109 & & 0.6 & 38.0 & 0.0 & 0.0 & 4.5 & 0.0 & 0.0 & 0.0 \\
\hline 110 & -1 & 0.960 & 0.0 & 0.0 & 0.0 & 58.5 & 0.0 & 5.5 & 0.0 \\
\hline BARRA110 & & -0.2 & 0.0 & 0.0 & 0.0 & 45.0 & 0.0 & 0.0 & 0.0 \\
\hline 111 & -1 & 0.972 & 0.0 & 0.0 & 0.0 & -54.0 & 0.0 & 0.0 & 0.0 \\
\hline BARRA111 & & 2.3 & 0.0 & 0.0 & 0.0 & 0.0 & 0.0 & 0.0 & 0.0 \\
\hline 112 & -1 & 0.952 & 0.0 & 0.0 & 0.0 & 102.0 & 0.0 & 0.0 & 0.0 \\
\hline BARRA112 & & -4.8 & 50.0 & 0.0 & 0.0 & 19.5 & 0.0 & 0.0 & 0.0 \\
\hline 113 & -1 & 0.983 & 0.0 & 0.0 & 0.0 & 9.0 & 0.0 & 0.0 & 0.0 \\
\hline BARRA113 & & -0.8 & 0.0 & 0.0 & 0.0 & 0.0 & 0.0 & 0.0 & 0.0 \\
\hline 114 & 0 & 0.965 & 0.0 & 0.0 & 0.0 & 12.0 & 0.0 & 0.0 & 0.0 \\
\hline BARRA114 & & -1.6 & 0.0 & 0.0 & 0.0 & 4.5 & 0.0 & 0.0 & 0.0 \\
\hline 115 & 0 & 0.964 & 0.0 & 0.0 & 0.0 & 33.0 & 0.0 & 0.0 & 0.0 \\
\hline BARRA115 & & -1.6 & 0.0 & 0.0 & 0.0 & 10.5 & 0.0 & 0.0 & 0.0 \\
\hline 116 & -1 & 1.001 & 0.0 & 0.0 & 0.0 & 0.0 & 0.0 & 0.0 & 0.0 \\
\hline BARRA116 & & 18.3 & -50.0 & 0.0 & 0.0 & 0.0 & 0.0 & 0.0 & 0.0 \\
\hline 117 & 0 & 0.974 & 0.0 & 0.0 & 0.0 & 30.0 & 0.0 & 0.0 & 0.0 \\
\hline BARRA117 & & -2.3 & 0.0 & 0.0 & 0.0 & 12.0 & 0.0 & 0.0 & 0.0 \\
\hline 118 & 0 & 0.952 & 0.0 & 0.0 & 0.0 & 49.5 & 0.0 & 0.0 & 0.0 \\
\hline BARRA118 & & 4.9 & 0.0 & 0.0 & 0.0 & 22.5 & 0.0 & 0.0 & 0.0 \\
\hline
\end{tabular}

RELATORIO DE CIRC. CA DO SIST. * AREA 1 *

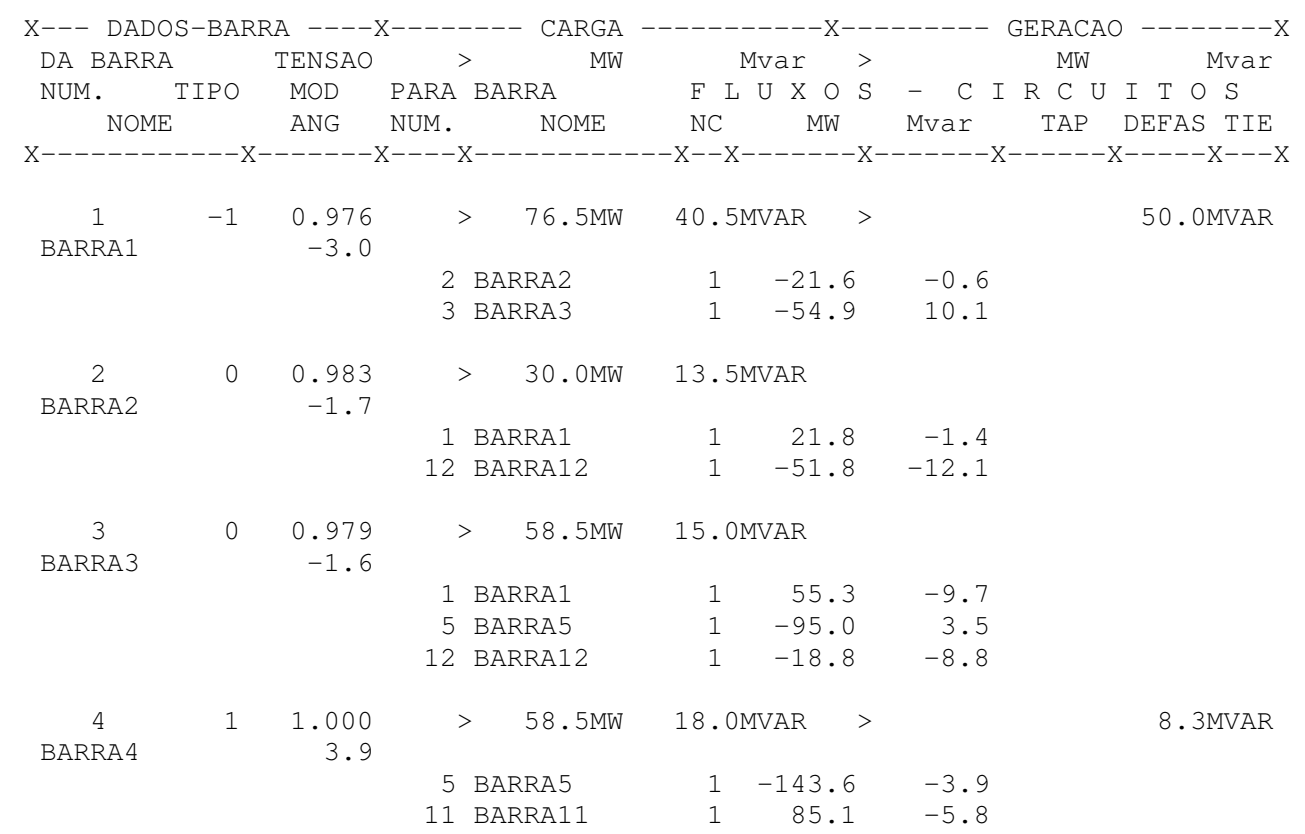




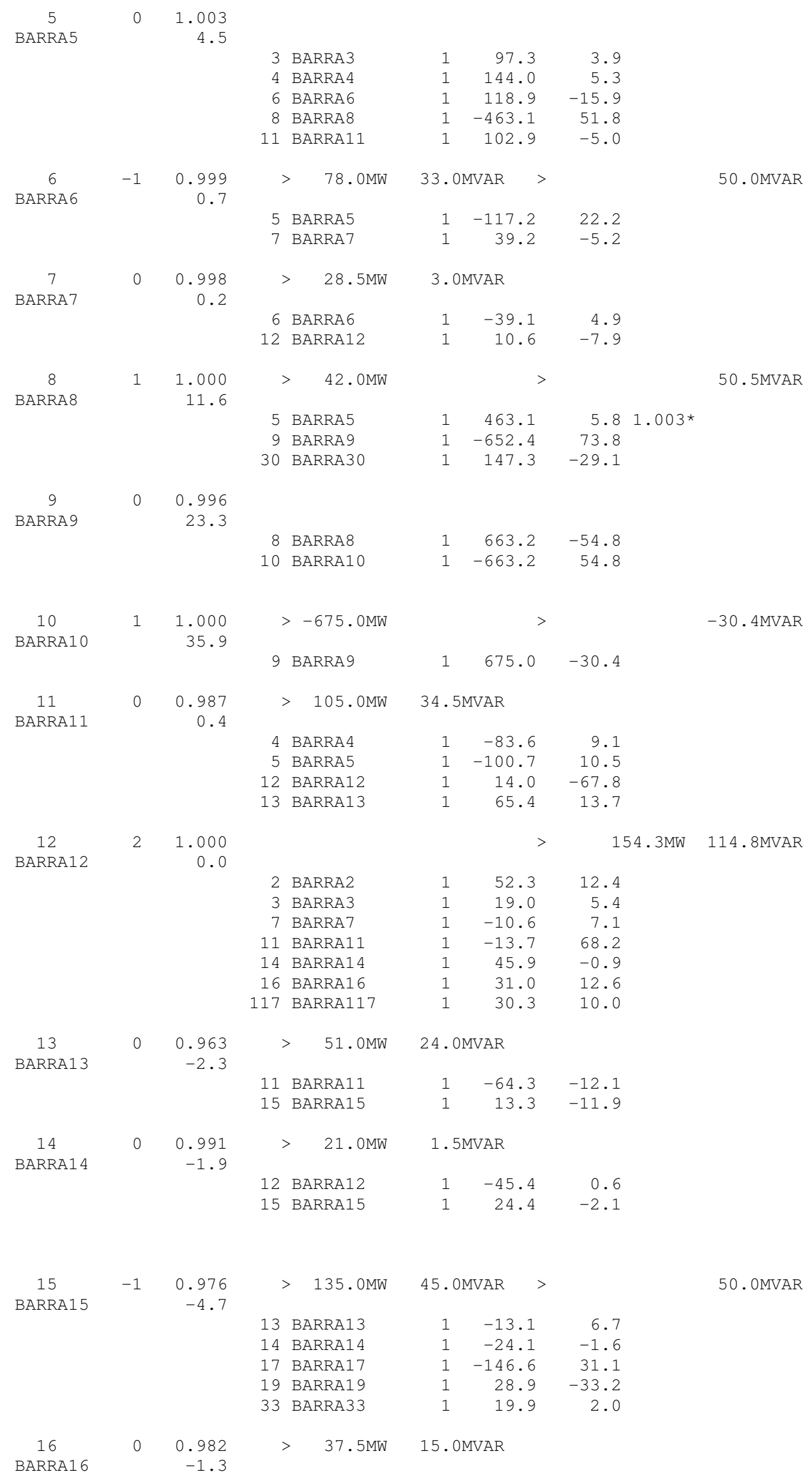




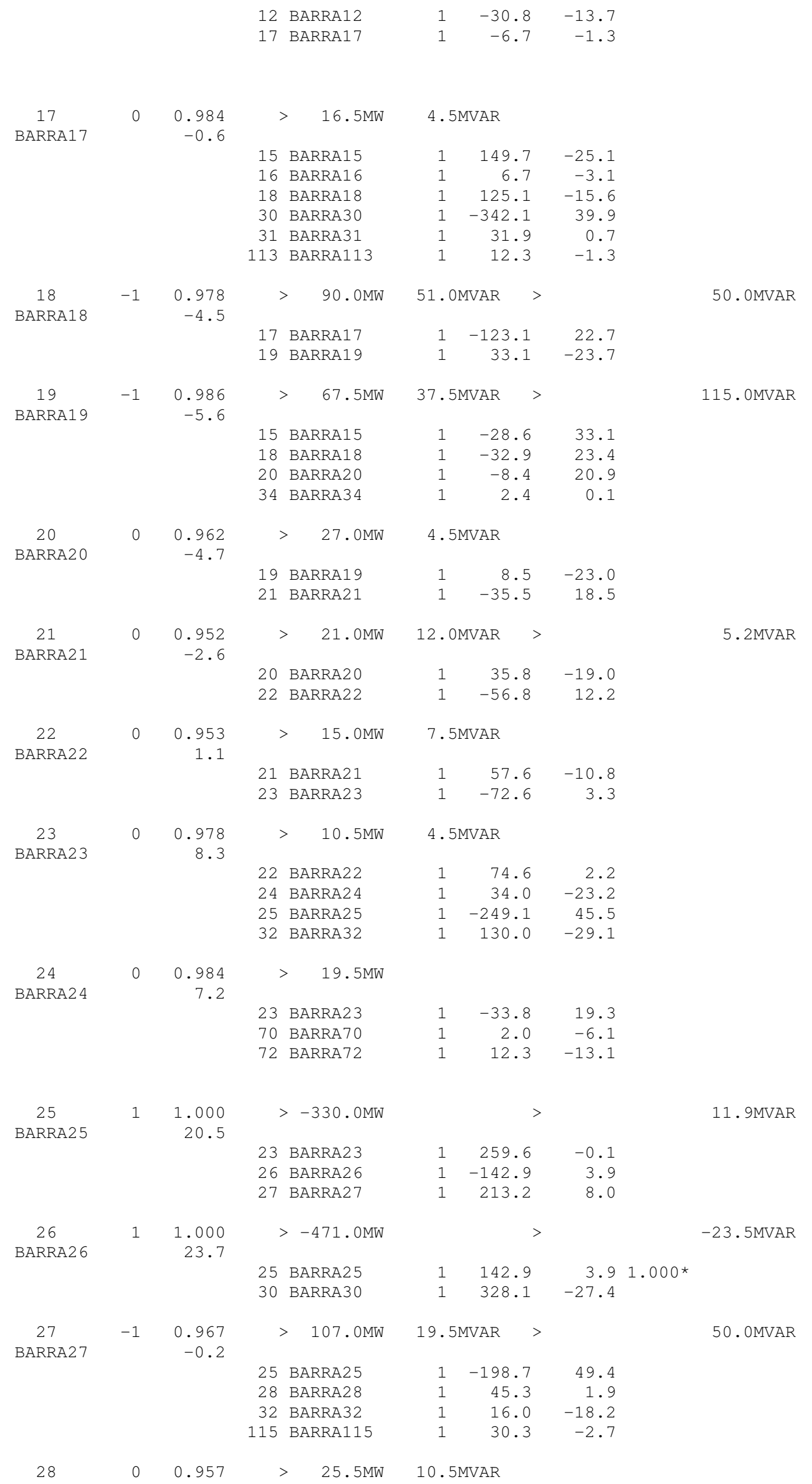




\begin{tabular}{|c|c|c|c|c|c|c|c|c|c|}
\hline BARRA2 8 & & -2.5 & $\begin{array}{l}27 \\
29\end{array}$ & $\begin{array}{l}\text { BARRA27 } \\
\text { BARRA29 }\end{array}$ & $\begin{array}{l}1 \\
1\end{array}$ & $\begin{array}{r}-44.9 \\
19.4\end{array}$ & $\begin{array}{l}-2.0 \\
-8.5\end{array}$ & & \\
\hline $\begin{array}{c}29 \\
\text { BARRA29 }\end{array}$ & 0 & $\begin{array}{r}0.959 \\
-3.8\end{array}$ & & $36.0 \mathrm{MW}$ & 6.01 & IVAR & & & \\
\hline & & & $\begin{array}{l}28 \\
31\end{array}$ & $\begin{array}{l}\text { BARRA28 } \\
\text { BARRA31 }\end{array}$ & $\begin{array}{l}1 \\
1\end{array}$ & $\begin{array}{l}-19.3 \\
-16.7\end{array}$ & $\begin{array}{r}6.8 \\
-12.8\end{array}$ & & \\
\hline $\begin{array}{c}30 \\
\text { BARRA30 }\end{array}$ & 0 & $\begin{array}{r}0.998 \\
7.3\end{array}$ & & & & & & & \\
\hline & & & 8 & BARRA 8 & 1 & -146.4 & -11.3 & & \\
\hline & & & 17 & BARRA 17 & 1 & 342.1 & 7.6 & $1.021 *$ & \\
\hline & & & 26 & BARRA2 6 & 1 & -319.5 & 28.6 & & \\
\hline & & & 38 & BARRA 38 & 1 & 123.7 & -25.0 & & \\
\hline 31 & -1 & 0.965 & & $54.0 \mathrm{MW}$ & 40.51 & IVAR > & & & $50.0 \mathrm{MVAR}$ \\
\hline BARRA31 & & -3.5 & & & & & & & \\
\hline & & & 17 & BARRA17 & 1 & -31.4 & -2.9 & & \\
\hline & & & 29 & BARRA29 & 1 & 16.8 & 12.2 & & \\
\hline & & & 32 & BARRA 32 & 1 & -39.4 & 0.2 & & \\
\hline 32 & -1 & 0.977 & & $88.5 \mathrm{MW}$ & 34.5 & IVAR > & & & 100.0MVAR \\
\hline BARRA32 & & -1.1 & & & & & & & \\
\hline & & & 23 & BARRA23 & 1 & -124.2 & 38.9 & & \\
\hline & & & 27 & BARRA27 & 1 & -15.9 & 16.8 & & \\
\hline & & & 31 & BARRA31 & 1 & 39.9 & -0.9 & & \\
\hline & & & 113 & BARRA113 & 1 & -3.2 & -4.3 & & \\
\hline & & & 114 & BARRA114 & 1 & 14.9 & 15.1 & & \\
\hline 33 & 0 & 0.964 & & $34.5 \mathrm{MW}$ & 13.5 & IVAR & & & \\
\hline & & -6.1 & 15 & BARRA 15 & 1 & -19.8 & -4.5 & & \\
\hline & & & 37 & BARRA37 & 1 & -14.7 & -9.0 & & \\
\hline 34 & 0 & 0.976 & & $88.5 \mathrm{MW}$ & 39.0 & IVAR & & & \\
\hline BARRA3 4 & & -5.8 & & & & & & & \\
\hline & & & 19 & BARRA19 & 1 & -2.4 & -6.2 & & \\
\hline & & & 36 & BARRA 36 & 1 & 44.5 & -13.1 & & \\
\hline & & & 37 & BARRA37 & 1 & -141.7 & -16.7 & & \\
\hline & & & 43 & BARRA 43 & 1 & 11.0 & 10.4 & & \\
\hline 35 & 0 & 0.975 & & $49.5 \mathrm{MW}$ & 13.5 & IVAR & & & \\
\hline BARRA35 & & -6.6 & & BARRA 36 & 1 & 2.2 & $-175^{-1}$ & & \\
\hline & & & $\begin{array}{l}36 \\
37\end{array}$ & $\begin{array}{l}\text { BARRA 36 } \\
\text { BARRA37 }\end{array}$ & $\begin{array}{l}1 \\
1\end{array}$ & $\begin{array}{r}2.2 \\
-51.7\end{array}$ & $\begin{array}{r}-11.5 \\
-2.0\end{array}$ & & \\
\hline 36 & -1 & 0.976 & & $46.5 \mathrm{MW}$ & 25.5 & IVAR & & & 50.0 MVAR \\
\hline BARRA 36 & & -6.6 & & & & & & & \\
\hline & & & 34 & BARRA 34 & 1 & -44.4 & 13.2 & & \\
\hline & & & 35 & BARRA35 & 1 & -2.1 & 11.3 & & \\
\hline 37 & 0 & 0.982 & & & & & & & \\
\hline BARRA 37 & & -5.0 & & & & & & & \\
\hline & & & 33 & BARRA 33 & 1 & 14.9 & 6.0 & & \\
\hline & & & 34 & BARRA 34 & 1 & 142.3 & 17.7 & & \\
\hline & & & 35 & BARRA 35 & 1 & 52.0 & 2.1 & & \\
\hline & & & 38 & BARRA38 & 1 & -377.6 & 44.5 & & \\
\hline & & & 39 & BARRA39 & 1 & 92.8 & -17.8 & & \\
\hline & & & 40 & BARRA 40 & 1 & 75.7 & -28.3 & & \\
\hline 38 & 0 & 0.997 & & & & & & & \\
\hline BARRA38 & & 3.5 & & & & & & & \\
\hline & & & 30 & BARRA30 & 1 & -123.0 & -8.7 & & \\
\hline & & & 37 & BARRA 37 & 1 & 377.6 & 11.8 & $1.022 *$ & \\
\hline & & & 65 & BARRA 65 & 1 & -254.6 & -3.1 & & \\
\hline 39 & 0 & 0.975 & & $>\quad 40.5 \mathrm{MW}$ & 16.5 & IVAR & & & \\
\hline BARRA39 & & -11.3 & & & & & & & \\
\hline & & & $\begin{array}{l}37 \\
40\end{array}$ & $\begin{array}{l}\text { BARRA } 37 \\
\text { BARRA } 40\end{array}$ & 1 & $\begin{array}{r}-89.8 \\
49.3\end{array}$ & $\begin{array}{r}25.0 \\
-41.5\end{array}$ & & \\
\hline 40 & -1 & 0.992 & & $99.0 \mathrm{MW}$ & 34.5 & IVAR & & & $150.0 \mathrm{MVAR}$ \\
\hline BARRA & & -13.5 & & & & & & & \\
\hline
\end{tabular}




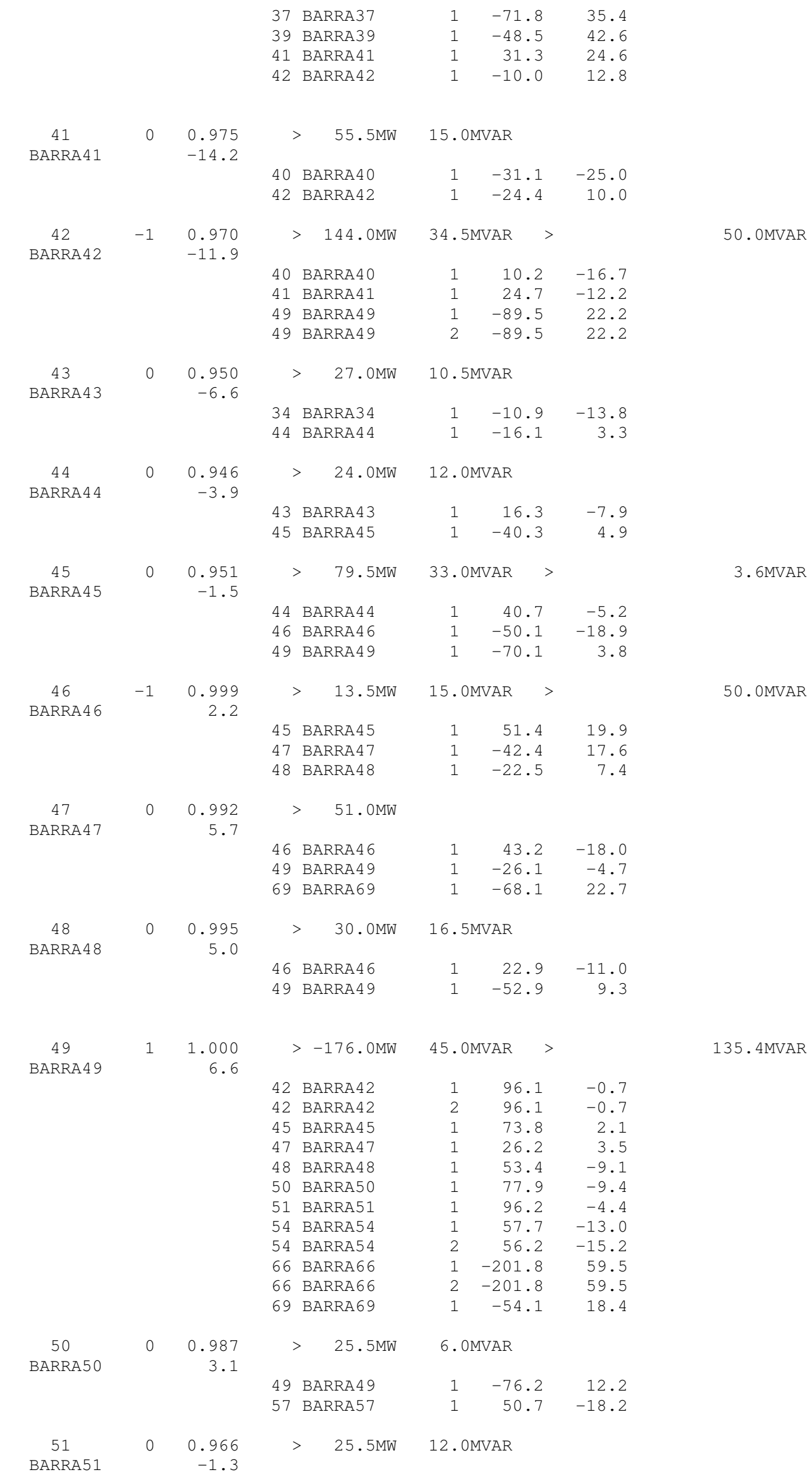




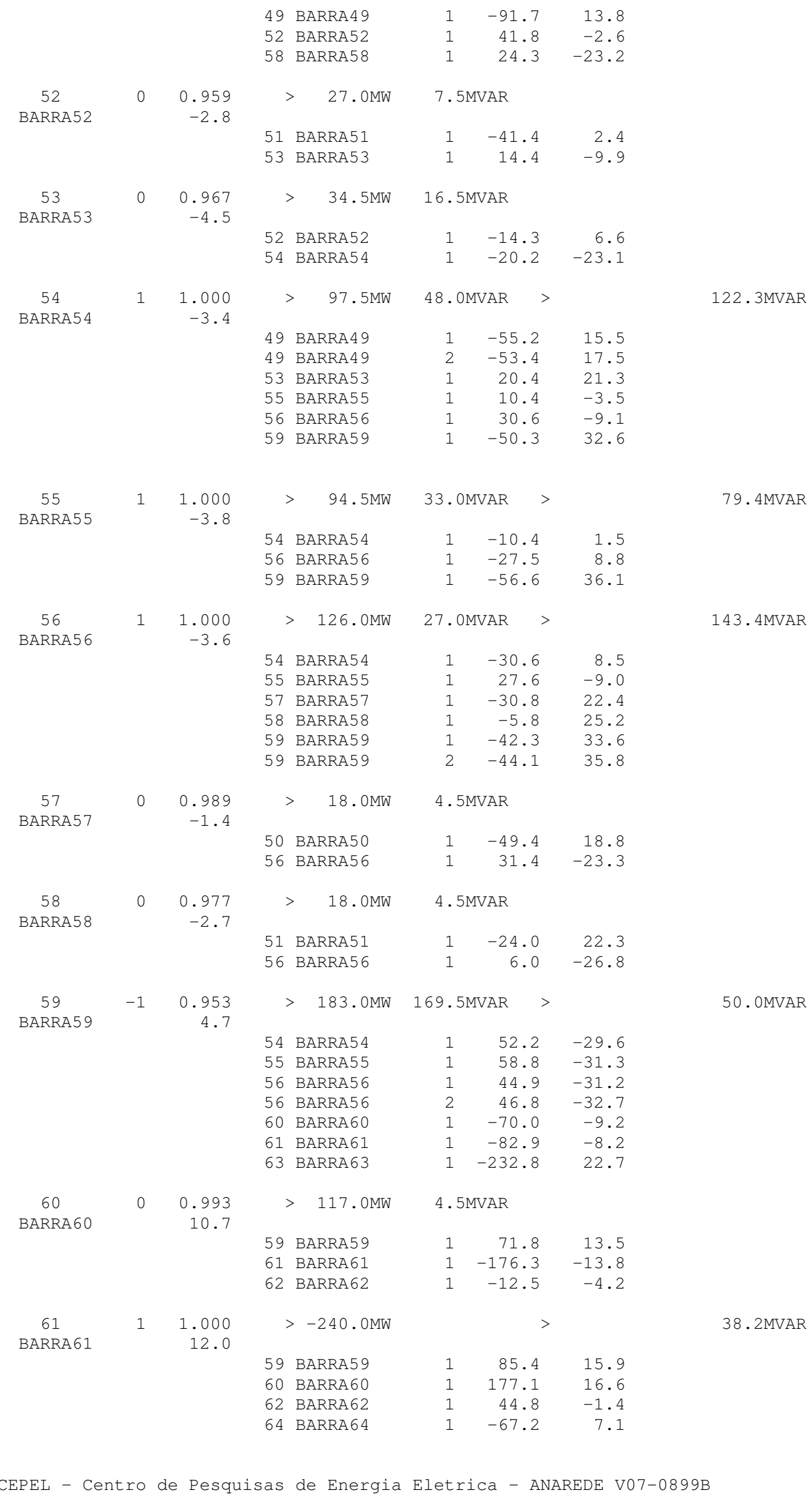




\begin{tabular}{|c|c|c|c|c|c|c|c|c|c|}
\hline \multirow[t]{5}{*}{$\begin{array}{c}62 \\
\text { BARRA } 62\end{array}$} & \multirow[t]{5}{*}{-1} & \multirow[t]{5}{*}{$\begin{array}{r}0.997 \\
11.1\end{array}$} & $>$ & $>\quad 116.0 \mathrm{MW}$ & \multicolumn{2}{|c|}{ 21. OMVAR } & \multicolumn{2}{|c|}{ s } & \multirow[t]{5}{*}{ 50. OMVAR } \\
\hline & & & 60 & BARRA 60 & 1 & 12.5 & 2.9 & & \\
\hline & & & 61 & BARRA 61 & 1 & -44.6 & 1.2 & & \\
\hline & & & 66 & BARRA 66 & 1 & -51.9 & 10.5 & & \\
\hline & & & 67 & BARRA 67 & 1 & -32.0 & 14.5 & & \\
\hline \multirow{4}{*}{$\begin{array}{c}63 \\
\text { BARRA } 63\end{array}$} & \multirow[t]{4}{*}{0} & \multirow{4}{*}{$\begin{array}{r}0.995 \\
10.4\end{array}$} & & & & & & & \\
\hline & & & & & & & & & \\
\hline & & & 59 & BARRA59 & 1 & 232.8 & 0.6 & $1.049 *$ & \\
\hline & & & 64 & BARRA 64 & 1 & -232.8 & -0.6 & & \\
\hline \multirow{5}{*}{$\begin{array}{c}64 \\
\text { BARRA } 64\end{array}$} & \multirow[t]{5}{*}{0} & \multirow{5}{*}{$\begin{array}{r}0.998 \\
13.1\end{array}$} & & & & & & & \\
\hline & & & & & & & & & \\
\hline & & & 61 & BARRA 61 & 1 & 67.2 & -5.9 & $1.000 *$ & \\
\hline & & & 63 & BARRA 63 & 1 & 233.8 & -9.9 & & \\
\hline & & & 65 & BARRA 65 & 1 & -301.0 & 15.8 & & \\
\hline 65 & 1 & 1.000 & $>$ & $>-587.0 \mathrm{MW}$ & & & & & $-120.9 \mathrm{MVAR}$ \\
\hline & & 18.3 & & & & & & & \\
\hline & & & 38 & BARRA 38 & 1 & 260.7 & -44.0 & & \\
\hline & & & 64 & BARRA 64 & 1 & 303.5 & -25.9 & & \\
\hline & & & 66 & BARRA 66 & 1 & 18.4 & 0.1 & $1.000 *$ & \\
\hline & & & 68 & BARRA 68 & 1 & 4.4 & -51.1 & & \\
\hline $\begin{array}{c}66 \\
\text { BARRA } 66\end{array}$ & 1 & 1.000 & & $>-530.0 \mathrm{MW}$ & 27.0 & MVAR & & & $-27.5 \mathrm{MVAR}$ \\
\hline & & & 49 & BARRA 49 & 1 & 209.7 & -21.1 & & \\
\hline & & & 49 & BARRA 49 & 2 & 209.7 & -21.1 & & \\
\hline & & & 62 & BARRA 62 & 1 & 53.3 & -9.9 & & \\
\hline & & & 65 & BARRA 65 & 1 & -18.4 & 0.1 & & \\
\hline & & & & BARRA 67 & 1 & 75.6 & -2.4 & & \\
\hline 67 & 0 & 0.987 & $>$ & $42.0 \mathrm{MW}$ & 10.5 & IVAR & & & \\
\hline & & 13.5 & & $B \cap P B \cap 62$ & 1 & 323 & $-16 \quad 0$ & & \\
\hline & & & $\begin{array}{l}62 \\
66\end{array}$ & $\begin{array}{l}\text { BARRA } 62 \\
\text { BARRA } 66\end{array}$ & 1 & $\begin{array}{r}32.3 \\
-74.3\end{array}$ & $\begin{array}{r}-10.0 \\
5.5\end{array}$ & & \\
\hline 68 & 0 & .003 & & & & & & & \\
\hline BARRA 68 & & 18.3 & & & & & & & \\
\hline & & & 65 & BARRA 65 & 1 & -4.4 & -12.8 & & \\
\hline & & & 69 & BARRA 69 & 1 & 12.6 & 4.2 & $1.001 *$ & \\
\hline & & & 81 & BARRA 81 & 1 & -8.2 & -25.0 & & \\
\hline & & & 116 & BARRA116 & 1 & 0.0 & 33.6 & & \\
\hline 69 & 1 & 1.000 & & $>-494.0 \mathrm{MW}$ & & & & & $-55.7 \mathrm{MVAR}$ \\
\hline BARRA69 & & 18.0 & 47 & BARRA 47 & 1 & 72.7 & $-14 \cdot 7$ & & \\
\hline & & & 49 & BARRA 49 & 1 & 57.5 & -15.5 & & \\
\hline & & & 68 & BARRA 68 & 1 & -12.6 & -4.1 & & \\
\hline & & & 70 & BARRA 70 & 1 & 151.5 & -16.4 & & \\
\hline & & & 75 & BARRA 75 & 1 & 154.2 & -11.7 & & \\
\hline & & & 77 & BARRA 77 & 1 & 70.8 & 6.8 & & \\
\hline 70 & -1 & 0.987 & $>$ & $99.0 \mathrm{MW}$ & 30.0 & IVAR & & & 50.0 MVAR \\
\hline BARRA 70 & & 6.6 & & & & & & & \\
\hline & & & 24 & BARRA2 4 & 1 & -2.0 & -3.8 & & \\
\hline & & & 69 & BARRA 69 & 1 & -144.6 & 33.7 & & \\
\hline & & & 71 & BARRA 71 & 1 & 14.9 & -22.9 & & \\
\hline & & & 74 & BARRA 74 & 1 & 28.3 & -0.7 & & \\
\hline & & & 75 & BARRA75 & 1 & 4.4 & 13.7 & & \\
\hline 71 & 0 & 0.994 & & & & & & & \\
\hline BARRA 71 & & 6.2 & & & & & & & \\
\hline & & & 70 & BARRA 70 & 1 & -14.9 & 22.3 & & \\
\hline & & & 72 & BARRA 72 & 1 & 5.8 & -6.9 & & \\
\hline & & & 73 & BARRA 73 & 1 & 9.0 & -15.4 & & \\
\hline 72 & 1 & 1.000 & $>$ & $>\quad 18.0 \mathrm{MW}$ & & & & & 11. 5MVAR \\
\hline & & & 24 & BARRA2 4 & 1 & -12.2 & 8.9 & & \\
\hline
\end{tabular}




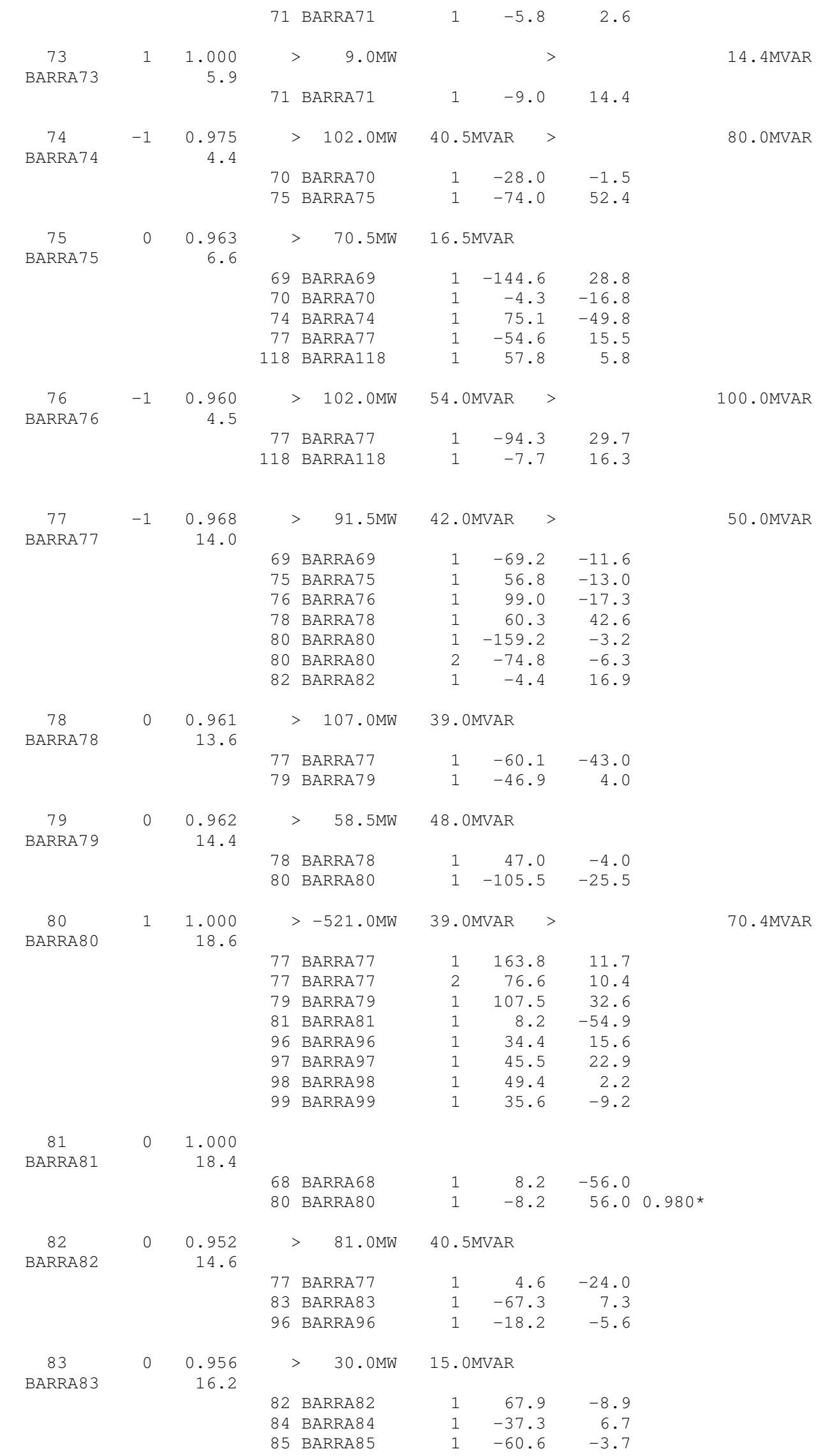




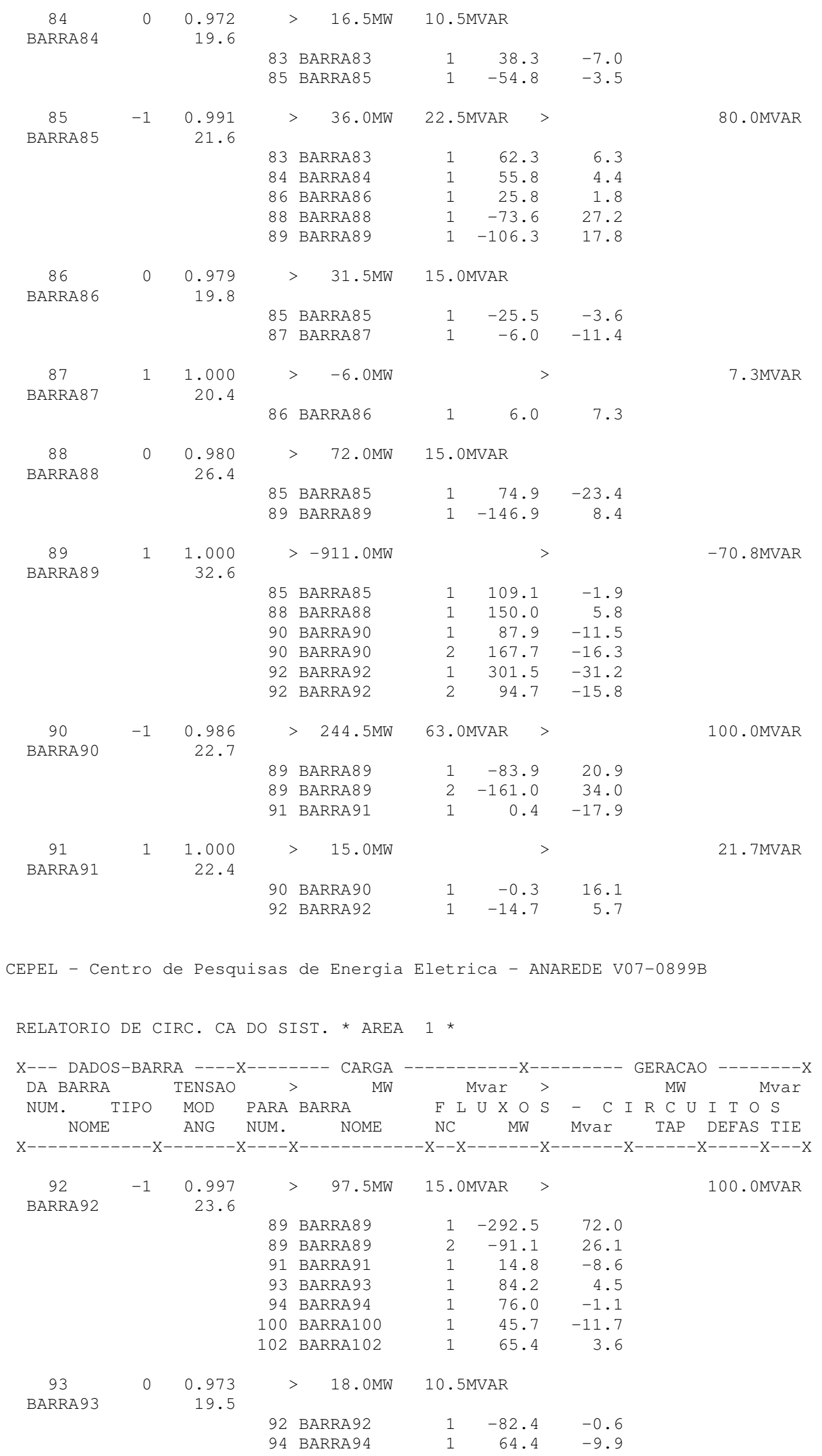




\begin{tabular}{|c|c|c|c|c|c|c|c|c|}
\hline $\begin{array}{c}94 \\
\text { BARRA94 }\end{array}$ & 0 & 0.966 & $x$ & $45.0 \mathrm{MW}$ & 24.0 & VAR & & \\
\hline & & & 92 & BARRA 92 & 1 & -73.2 & 6.4 & \\
\hline & & & 93 & BARRA 93 & 1 & -63.4 & 11.4 & \\
\hline & & & 95 & BARRA95 & 1 & 57.6 & 18.2 & \\
\hline & & & 96 & BARRA 96 & 1 & 25.1 & 2.0 & \\
\hline & & & 100 & BARRA 100 & 1 & 8.8 & -62.0 & \\
\hline 95 & 0 & 0.950 & > & $63.0 \mathrm{MW}$ & 46.5 & IVAR & & 19. OMVAR \\
\hline Dी & & 10.1 & 94 & BARRA 94 & 1 & -57.1 & -17.5 & \\
\hline & & & 96 & BARRA 96 & 1 & -5.9 & $-10 \cdot 0$ & \\
\hline 96 & 0 & 0.956 & $>$ & $57.0 \mathrm{MW}$ & 22.5 & IVAR & & \\
\hline BARRA96 & & 15.2 & & & & & & \\
\hline & & & 80 & BARRA 80 & 1 & -33.9 & -17.6 & \\
\hline & & & 82 & BARRA82 & 1 & 18.3 & 0.9 & \\
\hline & & & 94 & BARRA 94 & 1 & -24.9 & -3.5 & \\
\hline & & & 95 & BARRA 95 & 1 & 6.0 & 8.8 & \\
\hline & & & 97 & BARRA 97 & 1 & -22.4 & -11.0 & \\
\hline 97 & 0 & 0.970 & $y^{2}$ & $>\quad 22.5 \mathrm{MW}$ & 13.5 & IVAR & & \\
\hline BARRA97 & & 16.3 & & & & & & \\
\hline & & & 80 & BARRA 80 & 1 & -45.0 & -22.9 & \\
\hline & & & 96 & BARRA96 & 1 & 22.5 & 9.4 & \\
\hline 98 & 0 & 0.986 & $>$ & $51.0 \mathrm{MW}$ & 12.0 & IVAR & & \\
\hline BARRA98 & & 15.5 & & & & & & \\
\hline & & & $\begin{array}{r}80 \\
100\end{array}$ & $\begin{array}{l}\text { BARRA80 } \\
\text { BARRA100 }\end{array}$ & $\begin{array}{l}1 \\
1\end{array}$ & $\begin{array}{r}-48.8 \\
-2.2\end{array}$ & $\begin{array}{l}-2.3 \\
-9.7\end{array}$ & \\
\hline 99 & 1 & 1.000 & y & $63.0 \mathrm{MW}$ & & $>$ & & 11.9MVAR \\
\hline BARRA 99 & & 14.2 & & & & & & \\
\hline & & & 80 & BARRA 80 & 1 & -35.0 & 6.4 & \\
\hline & & & 100 & BARRA 100 & 1 & -28.0 & 5.5 & \\
\hline 100 & 1 & 1.000 & & $>-323.0 \mathrm{MW}$ & 27.0 & IVAR & & 67. 6MVAR \\
\hline BARRA 100 & & 15.6 & & & & & & \\
\hline & & & $\begin{array}{l}92 \\
94\end{array}$ & $\begin{array}{l}\text { BARRA } 92 \\
\text { BARRA } 94\end{array}$ & 1 & $\begin{array}{r}-44.3 \\
-8.2\end{array}$ & $\begin{array}{l}10.3 \\
58.4\end{array}$ & \\
\hline & & & 98 & BARRA 98 & $\begin{array}{l}1 \\
1\end{array}$ & $\begin{array}{l}0.2 \\
2.2\end{array}$ & $\begin{array}{r}5.4 \\
5.1\end{array}$ & \\
\hline & & & 99 & BARRA 99 & 1 & 28.2 & -7.0 & \\
\hline & & & 101 & BARRA101 & 1 & -23.2 & 23.7 & \\
\hline & & & 103 & BARRA103 & 1 & 187.3 & -49.1 & \\
\hline & & & 104 & BARRA1 04 & 1 & 87.1 & 3.3 & \\
\hline & & & 106 & BARRA10 6 & 1 & 93.8 & $-4 \cdot 1$ & \\
\hline 101 & 0 & 0.975 & x & $>\quad 33.0 \mathrm{MW}$ & 22.5 & IVAR & & \\
\hline BARRAIU & & & 100 & BARRA 100 & 1 & 23.5 & -25.4 & \\
\hline & & & 102 & BARRA 102 & 1 & -56.5 & 2.9 & \\
\hline 102 & 0 & 0.987 & > & 7. $5 \mathrm{MW}$ & 4.5 & IVAR & & \\
\hline BARRA102 & & 21.5 & & & & & & \\
\hline & & & 101 & $\begin{array}{l}\text { BARRA92 } \\
\text { BARRA1 } 01\end{array}$ & $\begin{array}{l}1 \\
1\end{array}$ & $\begin{array}{r}-64.9 \\
57.4\end{array}$ & $\begin{array}{l}-2.7 \\
-1.8\end{array}$ & \\
\hline 103 & 1 & 1.000 & & $>\quad-25.5 \mathrm{MW}$ & 24.0 & IVAR & & 100.4MVAR \\
\hline BARRA103 & & 9.5 & & & & & & \\
\hline & & & 100 & BARRA 100 & 1 & -181.4 & 63.3 & \\
\hline & & & 104 & BARRA104 & 1 & 49.4 & 7.7 & \\
\hline & & & 105 & BARRA105 & 1 & 65.8 & -2.5 & \\
\hline & & & 110 & BARRA110 & 1 & 91.7 & 7.9 & \\
\hline $\begin{array}{c}104 \\
\text { BARRA104 }\end{array}$ & -1 & 0.964 & $x$ & $>\quad 57.0 \mathrm{MW}$ & 37.5 & IVAR & & \\
\hline & & & 100 & BARRA 100 & 1 & -83.7 & 7.0 & \\
\hline & & & 103 & BARRA103 & 1 & -48.2 & -7.6 & \\
\hline & & & 105 & BARRA10 5 & 1 & 74.9 & -36.9 & \\
\hline $\begin{array}{c}105 \\
\text { BARRA105 }\end{array}$ & -1 & 0.971 & x & $46.5 \mathrm{MW}$ & 39.0 & VAR & & 20. OMVAR \\
\hline
\end{tabular}




\begin{tabular}{|c|c|c|c|c|c|c|c|c|}
\hline & & & $\begin{array}{l}103 \\
104 \\
106 \\
107 \\
108\end{array}$ & $\begin{array}{l}\text { BARRA103 } \\
\text { BARRA10 } 04 \\
\text { BARRA106 } \\
\text { BARRA107 } \\
\text { BARRA108 }\end{array}$ & $\begin{array}{l}1 \\
1 \\
1 \\
1 \\
1\end{array}$ & $\begin{array}{r}-63.5 \\
-74.1 \\
12.7 \\
40.6 \\
37.8\end{array}$ & $\begin{array}{r}5.6 \\
38.8 \\
-0.9 \\
-24.6 \\
-19.0\end{array}$ & \\
\hline $\begin{array}{l}106 \\
\text { BARRA106 }\end{array}$ & 0 & $\begin{array}{r}0.970 \\
2.7\end{array}$ & $\begin{array}{l}100 \\
105 \\
107\end{array}$ & $\begin{array}{l}\text { > } 64.5 \mathrm{MW} \\
\text { BARRA100 } \\
\text { BARRA105 } \\
\text { BARRA107 }\end{array}$ & $\begin{array}{l}1 \\
1 \\
1\end{array}$ & $\begin{array}{l}\text { IVAR > } \\
-88.5 \\
-12.7 \\
36.6\end{array}$ & $\begin{array}{r}18.2 \\
-0.4 \\
-24.7\end{array}$ & 17.2MVAR \\
\hline $\begin{array}{c}107 \\
\text { BARRA107 }\end{array}$ & -1 & $\begin{array}{r}0.996 \\
-1.9\end{array}$ & $\begin{array}{l}105 \\
106\end{array}$ & $\begin{array}{l}>\quad 75.0 \mathrm{MW} \\
\text { BARRA105 } \\
\text { BARRA106 }\end{array}$ & 18.0 & $\begin{array}{l}\text { IVAR > } \\
-39.4 \\
-35.6\end{array}$ & $\begin{array}{l}24.2 \\
23.7\end{array}$ & 60. OMVAR \\
\hline $\begin{array}{l}108 \\
\text { BARRA108 }\end{array}$ & 0 & $\begin{array}{r}0.975 \\
1.3\end{array}$ & $\begin{array}{l}105 \\
109\end{array}$ & $\begin{array}{l}\text { 3.0MW } \\
\text { BARRA105 } \\
\text { BARRA109 }\end{array}$ & $\begin{array}{l}1 \\
1\end{array}$ & $\begin{array}{l}\text { IVAR } \\
\begin{array}{r}-37.3 \\
34.3\end{array}\end{array}$ & $\begin{array}{r}18.5 \\
-20.0\end{array}$ & \\
\hline $\begin{array}{l}109 \\
\text { BARRA109 }\end{array}$ & 0 & $\begin{array}{r}0.977 \\
0.6\end{array}$ & $\begin{array}{l}108 \\
110\end{array}$ & $\begin{array}{l}>\quad 12.0 \mathrm{MW} \\
\text { BARRA108 } \\
\text { BARRA110 }\end{array}$ & $\begin{array}{l}1 \\
1\end{array}$ & $\begin{array}{l}\text { IVAR }> \\
-34.2 \\
22.2\end{array}$ & $\begin{array}{l}19.8 \\
13.7\end{array}$ & 38. OMVAR \\
\hline $\begin{array}{l}110 \\
\text { BARRA110 }\end{array}$ & -1 & $\begin{array}{r}0.960 \\
-0.2\end{array}$ & $\begin{array}{l}103 \\
109 \\
111 \\
112\end{array}$ & $\begin{array}{l}\text { 58.5MW } \\
\text { BARRA103 } \\
\text { BARRA109 } \\
\text { BARRA111 } \\
\text { BARRA112 }\end{array}$ & $\begin{array}{l}1 \\
1 \\
1 \\
1\end{array}$ & $\begin{array}{l}\text { IVAR } \\
-88.3 \\
-22.0 \\
-53.3 \\
105.1\end{array}$ & $\begin{array}{r}3.1 \\
-15.0 \\
0.5 \\
-28.0\end{array}$ & \\
\hline $\begin{array}{c}111 \\
\text { BARRA111 }\end{array}$ & -1 & $\begin{array}{r}0.972 \\
2.3\end{array}$ & 110 & $\begin{array}{l}>\quad-54.0 \mathrm{MW} \\
\text { BARRA110 }\end{array}$ & 1 & 54.0 & 0.0 & \\
\hline $\begin{array}{l}112 \\
\text { BARRA112 }\end{array}$ & -1 & $\begin{array}{r}0.952 \\
-4.8\end{array}$ & 110 & $\begin{array}{l}>\quad 102.0 \mathrm{MW} \\
\text { BARRA110 }\end{array}$ & 19.5 & $\begin{array}{l}\text { IVAR > } \\
-102.0\end{array}$ & 30.5 & 50.0 MVAR \\
\hline $\begin{array}{c}113 \\
\text { BARRA113 }\end{array}$ & -1 & $\begin{array}{r}0.983 \\
-0.8\end{array}$ & $\begin{array}{l}17 \\
32\end{array}$ & $\begin{array}{l}>\quad 9.0 \mathrm{MW} \\
\text { BARRA } 17 \\
\text { BARRA32 }\end{array}$ & $\begin{array}{l}1 \\
1\end{array}$ & $\begin{array}{r}-12.2 \\
3.2\end{array}$ & $\begin{array}{r}0.6 \\
-0.6\end{array}$ & \\
\hline $\begin{array}{l}114 \\
\text { BARRA114 }\end{array}$ & 0 & $\begin{array}{r}0.965 \\
-1.6\end{array}$ & $\begin{array}{r}32 \\
115\end{array}$ & $\begin{array}{l}>\quad 12.0 \mathrm{MW} \\
\text { BARRA } 32 \\
\text { BARRA115 }\end{array}$ & $\begin{array}{l}1 \\
1\end{array}$ & $\begin{array}{l}\text { IVAR } \\
\begin{array}{r}-14.8 \\
2.8\end{array}\end{array}$ & $\begin{array}{r}-16.3 \\
11.8\end{array}$ & \\
\hline $\begin{array}{c}115 \\
\text { BARRA115 }\end{array}$ & 0 & $\begin{array}{r}0.964 \\
-1.6\end{array}$ & $\begin{array}{r}2 \\
11\end{array}$ & $\begin{array}{l}>\quad 33.0 \mathrm{MW} \\
\text { BARRA } 27 \\
\text { BARRA114 }\end{array}$ & 10.5 & $\begin{array}{l}\text { IVAR } \\
\begin{array}{r}-30.2 \\
-2.8\end{array}\end{array}$ & $\begin{array}{r}1.6 \\
-12.1\end{array}$ & \\
\hline $\begin{array}{c}116 \\
\text { BARRA116 }\end{array}$ & -1 & $\begin{array}{r}1.001 \\
18.3\end{array}$ & 68 & BARRA 68 & 1 & 0.0 & -50.0 & $-50.0 \mathrm{MVAR}$ \\
\hline $\begin{array}{l}117 \\
\text { BARRA117 }\end{array}$ & 0 & $\begin{array}{r}0.974 \\
-2.3\end{array}$ & 12 & $\begin{array}{l}>\quad 30.0 \mathrm{MW} \\
\text { BARRA12 }\end{array}$ & 12.0 & $\begin{array}{l}\text { IVAR } \\
-30.0\end{array}$ & -12.0 & \\
\hline $\begin{array}{c}118 \\
\text { BARRA118 }\end{array}$ & 0 & $\begin{array}{r}0.952 \\
4.9\end{array}$ & $\begin{array}{l}75 \\
76\end{array}$ & $\begin{array}{l}>\quad 49.5 \mathrm{MW} \\
\text { BARRA } 75 \\
\text { BARRA } 76\end{array}$ & 22.5 & $\begin{array}{l}\text { IVAR } \\
\\
-57.3 \\
7.8\end{array}$ & $\begin{array}{r}-5.2 \\
-17.3\end{array}$ & \\
\hline
\end{tabular}


TOTAIS DA AREA 1

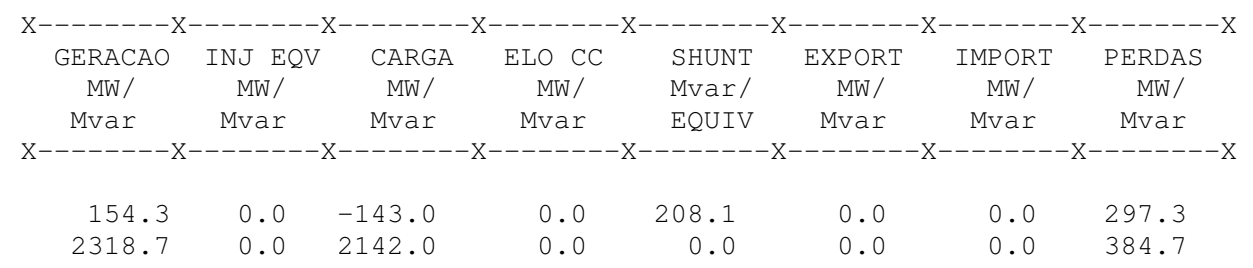

